\title{
Homotopy problems for harmonic maps to spaces of nonpositive curvature
}

\author{
Bent Fuglede
}

Dedicated to the memory of Professor Aurel Cornea

\begin{abstract}
Two results by N.J. Korevaar and R.M. Schoen (1993) about solvability of the free homotopy problem and the Dirichlet homotopy problem for harmonic maps from a compact Riemannian manifold $X$ to a compact geodesic space $Y$ of nonpositive curvature are strengthened here, and extended, particularly by allowing $X$ to be any compact admissible Riemannian polyhedron. In that setting, the two results were stated in the author's monograph from 2001 with J. Eells, but the short proofs given there were incomplete.
\end{abstract}

\section{Introduction}

As shown in the seminal paper by Eells and Sampson [10], if $X$ and $Y$ are compact Riemannian manifolds without boundary, and if $Y$ has nonpositive sectional curvature, then every smooth map $X \rightarrow Y$ is homotopic with a harmonic map which has minimum energy in its homotopy class. This was extended by Hamilton [20] to the case where $X$ has a smooth boundary $b X$, on which the maps shall be kept fixed throughout the homotopy - the so-called Dirichlet homotopy problem. Uniqueness results were obtained by Hartman [21]. While the stated results were established using the heat equation method, new proofs were given by Schoen [34] by a variational approach. For related results, see Schoen and Yau [39].

A new development was initiated by Gromov and Schoen [19], who extended the above results to the case of maps into a Riemannian space with singularities, more precisely, a compact Riemannian polyhedron of nonpositive curvature in the sense of A.D. Alexandrov, and embedded isometrically in some $\mathbb{R}^{N}$. In that setting (and in similar settings below), a harmonic map is defined to be a continuous locally energy minimizing map (see Remark 2.6 and Lemma 2.7 in the present paper for similar and, actually, equivalent definitions of harmonicity). Furthermore, local Lipschitz continuity of harmonic maps was established in [19]. These results were further extended 
by Korevaar and Schoen [25] to the still more general case of a compact geodesic space target $Y$, again of nonpositive Alexandrov curvature.

In the present paper we keep the compact geodesic space target $Y$ of nonpositive curvature. As source space we allow any compact Riemannian polyhedron which is admissible in the sense of $[9,18]$. Lipschitz continuity of harmonic maps must then necessarily be replaced by Hölder continuity; cf. [6], [9, Example 6.1].

In the case of free homotopy there is no prescribed boundary map, and a minimizing sequence of equivariant maps may diverge to infinity in the universal cover $\widetilde{Y}$ of $Y$; cf. [25, Remark 2.6.6]. We adapt (for Theorem 3.2) the impressive existence proof by Korevaar and Schoen of their result [25, Theorems 2.6.4 and 2.7.1] to the present case of a polyhedral domain $X$, but we do not require that $b X=\varnothing$. We further go beyond [25] by proving not only that, as expected from [10] and [19], every homotopy class $\mathcal{H}$ of continuous maps $X \rightarrow Y$ has an energy minimizer which is harmonic, but that indeed every energy minimizer in $\mathcal{H}$ is harmonic. As expected from Hartman [21], two energy minimizers in $\mathcal{H}$ are identical if they agree at a point. As an extension of another uniqueness result of [21] it is shown by Mese [30] that if $Y$ has Alexandrov curvature $\leqslant \kappa$ for some constant $\kappa<0$ (and if $X$ is a manifold) then an energy minimizer in $\mathcal{H}$ is unique unless its image is contained in a geodesic of $Y$.

For Hölder continuity of local energy minimizers we use [9] and [11], drawing on Jost [23]. We now obtain a more uniform estimate involving the energy (Proposition 3.1 and Remark 3.3). For Hölder continuity of harmonic maps in different settings, see $[17,29,36,38,41]$.

For the Dirichlet homotopy problem it is supposed that $b X \neq \varnothing$. We allow (in Theorem 4.1) $X \backslash b X$ to be replaced more generally (as domain of harmonicity of the solution) by any connected regular open set $\Omega \subset X$ such that $\Omega \subset X \backslash b X$, or at least that $\Omega \cap b X$ is not dense in $b X$. Regularity of $\Omega$ is understood in the sense of potential theory - a necessary, fairly mild local condition on $\partial \Omega$; and $X \backslash b X$ is itself always connected, open, and regular. We show that, as expected from Hamilton's theorem [20] and from [21], every homotopy class $\mathcal{H}$ of continuous maps $X \rightarrow Y$ which agree on $X \backslash \Omega$ with a prescribed continuous map $\chi: X \rightarrow Y$ of finite energy has a unique energy minimizer $\varphi_{0}$, and $\varphi_{0}$ is harmonic and Hölder continuous in $\Omega$. The existence of an energy minimizer $\varphi_{0}$ can be established in the same way as in the free homotopy case, as briefly explained in [25, p. 658] (where the source space $X$ is a compact Riemannian manifold with smooth boundary $\partial X$, and where $\Omega=X \backslash \partial X)$. Our existence proof of Theorem 4.1 (Dirichlet homotopy) is simpler and more elementary. By application of 
the Banach-Saks theorem it is shown that the Dirichlet boundary condition prevents a minimizing sequence of equivariant maps from escaping to infinity in the universal cover $\widetilde{Y}$. Continuity of the minimizer $\varphi_{0}$ up to the boundary of $\Omega$, and harmonicity in $\Omega$, are reduced to the same for the solution to the ordinary variational Dirichlet problem for maps into a simply connected space such as $\tilde{Y}$, as established in [14] (see also [16]) by use of potential theory with respect to the H. Cartan fine topology; this replaces the use of a result of Serbinowski [35] in [25, p. 658] for proving in their setting that $\varphi$ is Hölder continuous $C^{\alpha}$ up to the boundary (supposed smooth), provided that $\chi$ is so. When specialized to the smooth source manifold in [25, Theorem 2.7.2] our result involving continuous boundary data neither implies the quoted result from [25], nor is it implied thereby.

Theorem 3.2 (free homotopy) and Theorem 4.1 (for the particular case $\Omega=X \backslash b X)$ were both stated in [9, Theorems 11.1 and 11.2], but the proofs were incomplete. ${ }^{1}$ The case of the free homotopy problem when the source space is an admissible flat 2-complex was treated by Daskalopoulos and Mese [8].

It is understood in Theorems 3.2 and 4.1 that the Riemannian metric $g$ on the source polyhedron $X$ is simplex-wise smooth (as defined in [9, p. 151], cf. [19, p. 182]); this is needed for the extension in [9] of the energy concept of [25] (for maps from a Riemannian domain) to the present polyhedral source space $(X, g)$. However, when confined to maps into a (compact) smooth Riemannian manifold $Y$ of nonpositive sectional curvature, Theorems 3.2 and 4.1 remain valid with nearly the same proofs even when $g$ is allowed to be merely measurable; see Theorem 5.2. In that setting there is a wellknown concept of energy of maps $X \rightarrow Y$, defined for example in terms of an isometric embedding of $Y$ in some $\mathbb{R}^{N}$, as in Nash's theorem; see [9, Lemma 9.3]. The two energy concepts are indeed known to be identical if $g$ happens to be simplex-wise smooth [9, Theorem 9.2]. An application of Theorem 3.2 (with Riemannian manifold target) is given in $[24, \S 6]$.

For a survey of harmonic maps in the present setting, see [15].

\footnotetext{
${ }^{1}$ The main gap concerning $[9$, Theorem 11.1$]$ is the assertion that a minimizing sequence $\left(\tilde{\varphi}_{i}\right)$ of equivariant maps $\tilde{\varphi}_{i}: \widetilde{X} \rightarrow \widetilde{Y}$ has an $L^{2}$-convergent subsequence; but that may not be true, cf. [25, p. 647 and Remark 2.6.6.]

The same gap seems to be present already in $[28$, p. 63 , paragraph following (7.3)], despite a construction in [27], by which there remains the difficulty that equivariance of a sequence is not necessarily preserved under postcomposition with an automorphism. However, the result in question [27, Théorème 7.1] has been proved by different methods by Sacks and Uhlenbeck [33] and by Schoen and Yau [40] (and is covered by the present Theorem 3.2).
} 


\section{Preliminaries}

Throughout this article (unless otherwise specified), the source space $(X, g)$ is a compact Riemannian polyhedron (with possibly empty boundary $b X)$; cf. [19, p. 182; 9, Chapter 4]. This means that (i) $X$ is a compact connected metric space, (ii) there exists a finite (abstract) simplicial complex $K$ of finite dimension $m \geqslant 1$ and a bi-Lipschitz bijection $\theta$ of the (compact metric) space $(|K|, d)$ of the complex $K$ onto $X$, and (iii) for every simplex $s$ of $K$ there is given a smooth Riemannian metric $g_{s}$ on the geometric simplex $|s|$; we then write $g=\left(g_{s}\right)_{s \in K}$. The pair $(K, \theta)$ is called a triangulation of $X$. Recall, e.g., from [37, p. $110 \mathrm{f}$.] (or from [18]), that the space $|K|$ is the set of all maps $\xi$ of the set $K^{0}$ of vertices $v$ of $K$ into $[0,1]$ such that $\sum_{v \in K^{0}} \xi(v)=1$ and that $s:=\left\{v \in K^{0}: \xi(v)>0\right\}$ is an (abstract) simplex of $K$. The numbers $\xi(v)$ are called the barycentric coordinates of $\xi \in|K|$. A single vertex $v \in K^{0}$ is identified with the point $\xi$ of $|K|$ for which $\xi(v)=1$. The boundary $b K$ of $K$ is the union of those $(m-1)$-simplexes of $K$ which are faces of only one $m$-simplex, and $b X:=\theta(|b K|)$ is called the boundary of $X .|K|$ is a separable compact metric space with the barycentric metric $d$ defined by

$$
d\left(\xi, \xi^{\prime}\right)^{2}=\sum_{v \in K^{0}}\left(\xi(v)-\xi^{\prime}(v)\right)^{2}, \quad \xi, \xi^{\prime} \in|K| .
$$

We denote by $\mu=\mu_{g}$ the image under $\theta$ of the Riemannian volume measure on $(|K|, g)$ (that is, on each $m$-simplex $\left(|s|, g_{s}\right)$ of $\left.K\right)$. Furthermore, $d_{X}$ denotes the intrinsic Riemannian distance on $X$, induced by $\theta$ from the intrinsic Riemannian distance on $|K|$; cf. [9, p. 53]. (The intrinsic distance $d_{X}$ and the metric on $X$ induced by $\theta$ from the barycentric metric $d$ on $|K|$ are equivalent, but generally distinct.) In the sequel we often tacitly identify $|K|$ with $X=\theta(|K|)$ and a geometric simplex $|s|, s \in K$, with the 'curved' simplex $\theta(|s|) \subset X$.

Except in Propositions 2.1 and 2.2 we further require that $K$ and hence $X$ be admissible in the sense of $[9$, p. 45; 18]. This means that (i) $K$ is dimensionally $m$-homogeneous, i.e., every simplex of $K$ is a face of some $m$-simplex and (ii) $K$ is locally $(m-1)$-chainable (cf. $[6 ; 43, \S 2])$; i.e., for any simplex $\sigma$ of $K$, any two $m$-simplexes $s, t \in K$ containing $\sigma$ can be joined by a chain of $m$-simplexes $s=s_{0}, \ldots, s_{k}=t$ in $K$ containing $\sigma$ and such that $\operatorname{dim}\left(s_{i-1} \cap s_{i}\right)=m-1$ for $i \in\{1, \ldots, k\}$.

Every connected open subset $U$ of a polyhedron $X$ can be triangulated so as to become a polyhedron (not necessarily a subpolyhedron of $X$ ) such that every simplex of $U$ is a subset of some simplex of $X$. If $X$ is admissible then so is $U$; cf. [18]. When $X$ is Riemannian it is understood (in the absence 
of other indication) that $U$ is given the induced Riemannian metric. In particular, the open star st $v$ of a vertex $v$ of $X$ is an admissible polyhedron, and so is clearly the closed star $\overline{\mathrm{st}} v$.

The metric target space $\left(Y, d_{Y}\right)$ is required throughout (except in Section 5) to be a compact geodesic space of nonpositive Alexandrov curvature; see for example [2].

It is known that $\left(X, d_{X}\right)$ and $\left(Y, d_{Y}\right)$ are connected, path-connected and locally path-connected, and therefore have connected, complete, separable, metric, and locally compact universal covering spaces $\left(\widetilde{X}, d_{\widetilde{X}}\right)$ and $\left(\widetilde{Y}, d_{\widetilde{Y}}\right)$ with nonexpanding and locally isometric covering projections $p_{X}$ : $\widetilde{X} \rightarrow X$ and $p_{Y}: \widetilde{Y} \rightarrow Y$; cf. e.g. [31, $\left.\S 3.5\right],\left[37\right.$, p. 80]. Denote by $\Gamma_{X}$ and $\Gamma_{Y}$ the discrete groups of isometric covering transformations (deck transformations) of $\widetilde{X}$ and $\widetilde{Y}$, respectively. Thus $\Gamma_{X}$, respectively $\Gamma_{Y}$, is countable and isomorphic with the fundamental group $\pi_{1}(X)$, respectively $\pi_{1}(Y)$; cf. [37, Corollary 4 in $\left.\S 2.6\right]$.

Because $\widetilde{X} / \Gamma_{X}$ is homeomorphic with $X$ there exists a number $r_{X}>0$ such that

$$
p_{X} \text { maps } B_{\widetilde{X}}(\tilde{x}, \varepsilon) \text { isometrically onto } B_{X}(x, \varepsilon)
$$

for any $\tilde{x} \in \tilde{X}, x=p_{X}(\tilde{x})$ and $\varepsilon \leqslant r_{X}$. (We write for example $B_{X}(x, r)=$ $\left\{x^{\prime} \in X: d_{X}\left(x, x^{\prime}\right) \leqslant r\right\}, x \in X, r>0$.) See [31, Proposition 3.5.7], noting that $X$ is compact and $p_{X}$ a local isometry; similarly with $X, \widetilde{X}, r_{X}$ replaced by $Y, \widetilde{Y}, r_{Y}$. It follows that, say for any $\tilde{x} \in \widetilde{X}, p_{X}^{-1}\left(B_{X}(x, \varepsilon)\right)$ is the disjoint countable union of the closed balls $B_{\widetilde{X}}(\tilde{x}, \varepsilon), \tilde{x} \in p_{X}^{-1}(x)$, one for each $\gamma \in \Gamma_{X}$.

We may assume that every open star in $X$ (understood as the image under $\theta$ of the star of some vertex in $K)$ is a subset of some ball $B_{X}\left(x, r_{X}\right)$, $x \in X$; for that can always be arranged by passing to a subdivision of $X$ (that is, of $K$ ), as shown by Whitehead [42, Theorem 35, p. 317]; or see [37, Theorem 14 , p. 125]. Then $\left(\widetilde{X}, d_{\widetilde{X}}\right)$ becomes an admissible locally compact separable polyhedron whose $m$-simplexes $\tilde{s}$ are the connectivity components of $p_{X}^{-1}(s)$, with $s$ ranging over all $m$-simplexes of $X$. The polyhedron $\left(\widetilde{X}, d_{\widetilde{X}}\right)$ is a length space by $[9$, Proposition $4.1(\mathrm{~b})]$. Being complete and locally compact, $\left(\widetilde{X}, d_{\widetilde{X}}\right)$ is even a geodesic space $\left(\right.$ like $\left.\left(\widetilde{Y}, d_{\widetilde{Y}}\right)\right)$, according to the Hopf-Rinow theorem, cf. [2, p. 35; 31, p. 62]. Similarly, the compact length space $\left(X, d_{X}\right)$ is itself a geodesic space. The Riemannian metric $g_{s}$ on $s$ lifts uniquely to a Riemannian metric $\tilde{g}_{\tilde{s}}$ on $\tilde{s}$, and we thereby obtain an admissible, locally compact Riemannian polyhedron $(\widetilde{X}, \tilde{g})$.

After fixing a base point $x_{0} \in X$ and above it a point $\tilde{x}_{0} \in p_{X}^{-1}\left(x_{0}\right)$, a map $\varphi \in C(X, Y)$ (the continuous maps) lifts uniquely to a map $\tilde{\varphi} \in C(\widetilde{X}, \widetilde{Y})$ 
after choosing $\tilde{\varphi}\left(\tilde{x}_{0}\right) \in p_{Y}^{-1}\left(\varphi\left(x_{0}\right)\right)$, the lift $\tilde{\varphi}$ being then characterized by the lifting property

$$
\varphi \circ p_{X}=p_{Y} \circ \tilde{\varphi}
$$

cf. $[37$, p. 67$]$ or $[22$, p. 62$]$.

In the following two propositions and in Section 5 the Riemannian metric $g$ on $X$ is allowed to be just measurable (rather than simplex-wise smooth). Proposition 2.1 is basically well known, but we include a proof. (An equivalence class for some equivalence relation may be indicated by placing one of its elements between square brackets.)

Proposition 2.1. Let $(X, g)$ be a compact Riemannian polyhedron, and let $\left(Y, d_{Y}\right)$ be a compact geodesic space of nonpositive curvature. The set of homotopy classes $\mathcal{H}=[\varphi]$ of continuous maps $\varphi: X \rightarrow Y$ is mapped bijectively onto the set of conjugacy classes $\left[\varphi_{*}\right]$ of homomorphisms $\varphi_{*}: \Gamma_{X} \rightarrow$ $\Gamma_{Y}$ under the map $[\varphi] \mapsto\left[\varphi_{*}\right]$, whereby

$$
\tilde{\varphi} \circ \gamma=\varphi_{*}(\gamma) \circ \tilde{\varphi} \quad \text { in } \tilde{X} \text { for } \gamma \in \Gamma_{X}, \varphi \in \mathcal{H} .
$$

The covering transformation $\varphi_{*}(\gamma) \in \Gamma_{Y}$ is uniquely determined by (2.4) because $\tilde{\varphi}(\widetilde{X}) \neq \varnothing$ and because a covering transformation on $\tilde{Y}$ is determined by its action on any single point, $\widetilde{Y}$ being path-connected; cf. e.g. [22, p. 70]. If we replace the chosen point $\tilde{y}_{0}=\tilde{\varphi}\left(\tilde{x}_{0}\right) \in p_{Y}^{-1}\left(y_{0}\right)$ by another such point, that is, by $\beta\left(\tilde{y}_{0}\right)$ for some $\beta \in \Gamma_{Y}$, then $\tilde{\varphi}$ will be replaced by $\beta \circ \tilde{\varphi}$, and hence $\varphi_{*}(\gamma)$ by its conjugate $\beta \circ \tilde{\varphi} \circ \beta^{-1}$. The elements of the conjugacy class $\left[\varphi_{*}\right]$ thus correspond to the possible choices of $\tilde{y}_{0} \in p_{Y}^{-1}\left(y_{0}\right)$. For any other map $\psi$ from the given homotopy class $\mathcal{H}$ the corresponding homomorphism $\psi_{*}$ is, by the proposition, a conjugate to $\varphi_{*}$ and, therefore, equal to $\varphi_{*}$ by a certain choice of $\tilde{\varphi}\left(\tilde{x}_{0}\right)$ or $\tilde{\psi}\left(\tilde{x}_{0}\right)$.

Proof of Proposition 2.1. The polyhedron $X$ being a $C W$-complex (cf. [37, p. 400]) we may derive Proposition 2.1 from the corresponding result [37, Theorem 11 in $\S 8.1]$, in which $\Gamma_{X}$ and $\Gamma_{Y}$ are replaced by the fundamental groups $\pi_{1}\left(X, x_{0}\right)$ and $\pi_{1}\left(Y, y_{0}\right)$, respectively. The homomorphisms $\varphi_{*}: \Gamma_{X} \rightarrow \Gamma_{Y}$ shall then be replaced by the usual induced homomorphisms $\varphi_{\#}: \pi_{1}\left(X, x_{0}\right) \rightarrow \pi_{1}\left(Y, y_{0}\right), y_{0}=\varphi\left(x_{0}\right)$, given by

$$
\varphi_{\#}([\omega])=[\varphi \circ \omega] \text { for loops } \omega \text { in }\left(X, x_{0}\right) .
$$

Denote by $\mathcal{P}\left(\widetilde{X} ; \tilde{x}_{0}, \tilde{x}_{1}\right)$ the set of (continuous) paths in $\widetilde{X}$ from $\tilde{x}_{0}$ to $\tilde{x}_{1}$, and similarly relative to $\widetilde{Y}$. (Loops in $\left(X, x_{0}\right)$ then correspond to $\tilde{x}_{0}=\tilde{x}_{1}$.) 
The natural isomorphism $\psi_{X}: \Gamma_{X} \rightarrow \pi_{1}\left(X, x_{0}\right)$ is then given by

$$
\psi_{X}(\gamma)=\left[p_{X} \circ \tilde{\omega}: \tilde{\omega} \in \mathcal{P}\left(\tilde{X} ; \tilde{x}_{0}, \gamma\left(\tilde{x}_{0}\right)\right)\right], \quad \gamma \in \Gamma_{X}
$$

See [37, pp. 85-87], and note that $p_{X} \circ \tilde{\omega}$ indeed is a loop in $\left(X, x_{0}\right)$ since $p_{X}\left(\gamma\left(\tilde{x}_{0}\right)\right)=p_{X}\left(\tilde{x}_{0}\right)=x_{0}$; similarly for $\psi_{Y}: \Gamma_{Y} \rightarrow \pi_{1}\left(Y, y_{0}\right)$. It remains to evaluate

$$
\varphi_{*}:=\psi_{Y}^{-1} \circ \varphi_{\#} \circ \psi_{X} .
$$

For $\gamma \in \Gamma_{X}$ we have $\left(\right.$ since $\left.\tilde{\varphi}\left(\tilde{x}_{0}\right)=\tilde{y}_{0}\right)$

$$
\left(\psi_{Y} \circ \varphi_{*}\right)(\gamma)=\psi_{Y}\left(\varphi_{*}(\gamma)\right)=\left[p_{Y} \circ \tilde{\chi}: \tilde{\chi} \in \mathcal{P}\left(\tilde{Y} ; \tilde{y}_{0},\left(\varphi_{*}(\gamma) \circ \tilde{\varphi}\right)\left(\tilde{x}_{0}\right)\right)\right]
$$

(a homotopy class of loops in $\left.\left(Y, y_{0}\right)\right)$. Furthermore, by (2.3) and (2.5),

$$
\begin{aligned}
\left(\varphi_{\#} \circ \psi_{X}\right)(\gamma) & =\varphi_{\#} \circ\left[p_{X} \circ \tilde{\omega}: \tilde{\omega} \in \mathcal{P}\left(\tilde{X} ; \tilde{x}_{0}, \gamma\left(\tilde{x}_{0}\right)\right)\right] \\
& =\left[\varphi \circ p_{X} \circ \tilde{\omega}: \tilde{\omega} \in \mathcal{P}\left(\tilde{X} ; \tilde{x}_{0}, \gamma\left(\tilde{x}_{0}\right)\right)\right] \\
& =\left[p_{Y} \circ \tilde{\varphi} \circ \tilde{\omega}: \tilde{\omega} \in \mathcal{P}\left(\tilde{X} ; \tilde{x}_{0}, \gamma\left(\tilde{x}_{0}\right)\right)\right] \\
& \subset\left[p_{Y} \circ \tilde{\omega}^{\prime}: \tilde{\omega}^{\prime} \in \mathcal{P}\left(\tilde{Y} ; \tilde{y}_{0},(\tilde{\varphi} \circ \gamma)\left(\tilde{x}_{0}\right)\right)\right]
\end{aligned}
$$

The inclusion stems from $\tilde{\omega}^{\prime}:=\tilde{\varphi} \circ \tilde{\omega} \in \mathcal{P}\left(\tilde{Y} ; \tilde{y}_{0}, \tilde{\varphi}\left(\gamma\left(\tilde{x}_{0}\right)\right)\right)$ when $\tilde{\omega} \in$ $\mathcal{P}\left(\tilde{X} ; \tilde{x}_{0}, \gamma\left(\tilde{x}_{0}\right)\right)$. Note that $p_{Y} \circ \tilde{\omega}^{\prime}$ is a loop in $\left(Y, y_{0}\right)$ because $p_{Y}\left(\tilde{\varphi}\left(\gamma\left(\tilde{x}_{0}\right)\right)\right)$ $=\varphi\left(p_{X}\left(\gamma\left(\tilde{x}_{0}\right)\right)\right)=\varphi\left(p_{X}\left(\tilde{x}_{0}\right)\right)=\varphi\left(x_{0}\right)=y_{0}$. From (2.6) and (2.7) (whose left-hand members are equal) we have thus altogether obtained

$$
\begin{aligned}
& {\left[p_{Y} \circ \tilde{\chi}: \tilde{\chi} \in \mathcal{P}\left(\tilde{Y} ; \tilde{y}_{0},\left(\varphi_{*}(\gamma) \circ \tilde{\varphi}\right)\left(\tilde{x}_{0}\right)\right)\right]} \\
& \quad=\left[p_{Y} \circ \tilde{\omega}^{\prime}: \tilde{\omega}^{\prime} \in \mathcal{P}\left(\tilde{Y} ; \tilde{y}_{0},(\tilde{\varphi} \circ \gamma)\left(\tilde{x}_{0}\right)\right)\right]
\end{aligned}
$$

because inclusion between homotopy classes (of paths) implies equality. Thus every loop $\chi:=p_{Y} \circ \tilde{\chi}$ as in $(2.6)$ is homotopic (in $\left.\left(\underset{Y}{Y}, y_{0}\right)\right)$ with any loop $\omega^{\prime}:=p_{Y} \circ \tilde{\omega}^{\prime}$ as in (2.7). The lifted paths $\tilde{\chi}$ and $\tilde{\omega}^{\prime}$ in $\tilde{Y}$ have the same initial point $\tilde{y}_{0}$ and therefore also the same endpoint, so (2.4) holds true at $\tilde{x}_{0}$. For any $\tilde{x} \in \widetilde{X}$ we have $p_{Y}\left(\left(\varphi_{*}(\gamma) \circ \tilde{\varphi}\right)(\tilde{x})\right)=p_{Y}(\tilde{\varphi}(\tilde{x}))=\varphi\left(p_{X}(\tilde{x})\right)=$ $\varphi\left(p_{X}(\gamma(\tilde{x}))\right)=p_{Y}((\tilde{\varphi} \circ \gamma)(\tilde{x}))$; cf. (2.3). Consequently, (2.4) holds for any $\tilde{x} \in \tilde{X}$, by the unique lifting property $(2.3)$.

Proposition 2.2. Let $(X, g)$ be a compact Riemannian polyhedron, and let $\left(Y, d_{Y}\right)$ be a compact geodesic space of nonpositive curvature.

(a) $\operatorname{Lip}(X, Y)$ (Lipschitz maps) is uniformly dense in $C(X, Y)$ (continuous maps). 
(b) For any map $\varphi \in C(X, Y)$ and any number $r>0$ there exists a map $f \in C(X \times[0,1], Y)$ such that $f(x, 0)=\varphi(x)$ for $x \in X$, that $d_{Y}(f(x, t)$, $\varphi(x))<r$ for $(x, t) \in X \times[0,1]$, and that $f(\cdot, t) \in \operatorname{Lip}(X, Y)$ for each $t \in] 0,1]$.

In particular, every homotopy class $\mathcal{H} \subset C(X, Y)$, as in Proposition 2.1, contains a Lipschitz map. For $Y$ simply connected (and not necessarily compact) Proposition 2.2 was obtained in [14, Proposition 6], inspired by [25, p. 645] where ball averages are used. For the present polyhedral domain $X$, barycentric averages are preferable; see (2.10) below. The choice of Riemannian metric $g$ on $X$ in Proposition 2.2 is immaterial, by [9, Lemma $4.2]$.

Proof of Proposition 2.2. (a) Given $\varphi \in C(X, Y)$, consider a lift $\tilde{\varphi} \in C(\tilde{X}$, $\widetilde{Y})$; cf. (2.3). For any prescribed $\varepsilon>0$ with $\varepsilon \leqslant r_{X}$ from $(2.2)$, choose $\delta=$ $\delta(\varepsilon)>0$ so that $\delta \leqslant r_{X}$ and, by uniform continuity of $\varphi$,

$$
d_{Y}\left(\varphi(x), \varphi\left(x^{\prime}\right)\right)<\varepsilon \quad \text { for } x, x^{\prime} \in X \text { with } d_{X}\left(x, x^{\prime}\right) \leqslant \delta .
$$

We proceed to show that (with the same $\varepsilon$ and $\delta$ )

$$
d_{\widetilde{Y}}\left(\tilde{\varphi}(\tilde{x}), \tilde{\varphi}\left(\tilde{x}^{\prime}\right)\right) \leqslant \varepsilon \quad \text { for } \tilde{x}, \tilde{x}^{\prime} \in \tilde{X} \text { with } d_{\widetilde{X}}\left(\tilde{x}, \tilde{x}^{\prime}\right) \leqslant \delta .
$$

Consider the constant-speed geodesic $\tilde{\varrho}:\left[0, d_{\widetilde{X}}\left(\tilde{x}, \tilde{x}^{\prime}\right)\right] \rightarrow \widetilde{X}$ joining $\tilde{x}$ to $\tilde{x}^{\prime}$, and the image $\tilde{\varphi} \circ \tilde{\varrho}$, a path in $\tilde{Y}$. If $d_{\tilde{Y}}\left(\tilde{\varphi}(\tilde{x}), \tilde{\varphi}\left(\tilde{x}^{\prime}\right)\right)>\varepsilon$ there exists $\tau$, $0<\tau<d_{\tilde{X}}\left(\tilde{x}, \tilde{x}^{\prime}\right)$, such that $d_{\widetilde{Y}}(\tilde{\varphi}(\tilde{x}), \tilde{\varphi}(\tilde{\varrho}(\tau)))=\varepsilon$. From (2.2) and (2.3) we obtain, writing $p_{X}(\tilde{x})=x$ and $p_{X}(\tilde{\varrho}(\tau))=\varrho(\tau)$,

$$
d_{Y}(\varphi(x), \varphi(\varrho(\tau)))=d_{\tilde{Y}}(\tilde{\varphi}(\tilde{x}), \tilde{\varphi}(\tilde{\varrho}(\tau)))=\varepsilon .
$$

This contradicts $(2.8)$ (applied with $x^{\prime}$ replaced by $\left.\varrho(\tau)\right)$ because

$$
d_{X}(x, \varrho(\tau)) \leqslant d_{\widetilde{X}}(\tilde{x}, \tilde{\varrho}(\tau))<d_{\widetilde{X}}\left(\tilde{x}, \tilde{x}^{\prime}\right) \leqslant \delta
$$

$p_{X}$ being nonexpanding.

We may assume that, for every simplex $s \in K, \theta(|s|)$ has diameter $\leqslant \delta$ with $\delta$ from (2.8). (If this is not the case from the beginning we subdivide $K$ into simplexes $s^{\prime}$ such that $\left|s^{\prime}\right| \subset \theta^{-1}(B)$ for some open ball $B \subset X$ of diameter $\delta$; this is possible according to the abovementioned theorem of Whitehead [42] applied to a cover of $|K|$ by pre-images $\theta^{-1}(B)$ of open balls $B \subset X$ of diameter $\delta$ covering $X$ ). 
We proceed to adapt the construction from the proof of $[14$, Proposition $6(\mathrm{a})]$ (in which $Y$ was simply connected) to the lifted map $\tilde{\varphi}: \widetilde{X} \rightarrow \widetilde{Y}$. Given a point $\tilde{x} \in \tilde{X}$ with projection $p_{X}(\tilde{x})=x \in X$, the corresponding point $\xi=$ $\theta^{-1}(x) \in|K|$ has barycentric coordinates $\xi(v), v \in K^{0}$, and we have the probability measure

$$
\nu_{x}=\sum_{v \in K^{0}} \xi(v) \varepsilon_{\theta(v)}
$$

on $X$, where $\varepsilon_{p}$ denotes the mass 1 at a point $p \in X$. The support of $\nu_{x}$ is the simplex $\theta(|s|)$ in $X$, where $s$ denotes the abstract simplex in $K$ whose vertices are those $v \in K^{0}$ for which $\xi(v)>0$, so supp $\nu_{x} \subset B_{X}(x, \delta)$. Because $\delta \leqslant r_{X}$ (cf. the line following $\left.(2.2)\right), p_{X}$ maps $B_{\widetilde{X}}(\tilde{x}, \delta)$ isometrically onto $B(x, \delta)$, and $\nu_{x}$ therefore lifts to the probability measure

$$
\nu_{\tilde{x}}=\sum_{v \in K^{0}} \xi(v) \varepsilon_{\tilde{\theta}(v)}
$$

on $\tilde{X}$, whereby $\tilde{\theta}(v) \in B_{\widetilde{X}}(\tilde{x}, \delta)$ is defined by having the projection $\theta(v) \in$ $B_{X}(x, \delta)$ when $\xi(v)>0$.

Invoking [25, Lemma 2.5.1] we define

$$
\tilde{f}(\tilde{x})=\tilde{\varphi}_{\nu_{\tilde{x}}}, \quad \tilde{x} \in \tilde{X}
$$

$\tilde{\varphi}_{\nu_{\tilde{x}}}$ denoting the average (or centre of mass) of $\tilde{\varphi}: \widetilde{X} \rightarrow \widetilde{Y}$ with respect to $\nu_{\tilde{x}}$; that is, $\tilde{\varphi}_{\nu_{\tilde{x}}}$ is the unique point of $\widetilde{Y}$ which minimizes $\int_{\widetilde{X}} d_{\widetilde{Y}}^{2}\left(\tilde{\varphi}\left(\tilde{x}^{\prime}\right), \tilde{y}\right) d \nu_{\tilde{x}}\left(\tilde{x}^{\prime}\right)$ as a function of $\tilde{y} \in \widetilde{Y}$. For points $\tilde{x}, \tilde{x}^{\prime} \in \widetilde{X}$ with $d_{\widetilde{X}}\left(\tilde{x}, \tilde{x}^{\prime}\right) \leqslant \delta$ we then have $\tilde{\varphi}\left(\tilde{x}^{\prime}\right) \in B_{\widetilde{Y}}(\tilde{\varphi}(\tilde{x}), \varepsilon)$, by (2.9), and hence by [25, Proposition 2.4], or see $\left[9\right.$, Lemma 10.4], writing $\tilde{f}=\tilde{f}^{\varepsilon}$ to indicate the dependence of $\tilde{f}$ on $\varepsilon$ :

$$
\tilde{f}(\tilde{x})=\tilde{f}^{\varepsilon}(\tilde{x})=\tilde{\varphi}_{\nu_{\tilde{x}}} \in B_{\widetilde{Y}}(\tilde{\varphi}(\tilde{x}), \varepsilon)
$$

closed balls $B_{\widetilde{Y}}(\tilde{y}, \varepsilon)$ in $\left(\widetilde{Y}, d_{\tilde{Y}}\right)$ being convex. Thus, $\tilde{f}^{\varepsilon} \rightarrow \tilde{\varphi}$ uniformly in $\widetilde{X}$ as $\varepsilon \rightarrow 0$.

We show that $\tilde{f}=\tilde{f}^{\varepsilon} \in \operatorname{Lip}(\tilde{X}, \tilde{Y})$. Consider two points $\tilde{x}, \tilde{x}^{\prime}$ of $\tilde{X}$ with $d_{\widetilde{X}}\left(\tilde{x}, \tilde{x}^{\prime}\right) \leqslant \delta$, and write $p_{X}(\tilde{x})=x=\theta(\xi)$ and $p_{X}\left(\tilde{x}^{\prime}\right)=x^{\prime}=\theta\left(\xi^{\prime}\right)$ with $\xi, \xi^{\prime} \in|K|$ and $d_{X}\left(x, x^{\prime}\right) \leqslant \delta$. Apply [25, Proposition 2.5.2] (with $\alpha=1$ ) to the corresponding representing measures $\nu_{\tilde{x}}$ and $\nu_{\tilde{x}^{\prime}}$; cf. (2.10). After 
division by $d_{\tilde{Y}}\left(\tilde{f}(\tilde{x}), \tilde{f}\left(\tilde{x}^{\prime}\right)\right)$ (if nonzero) we obtain by $(2.11)$ with the above notation

$$
\begin{aligned}
d_{\widetilde{Y}}\left(\tilde{f}(\tilde{x}), \tilde{f}\left(\tilde{x}^{\prime}\right)\right) & \leqslant 2 \int_{\widetilde{X}} d_{\widetilde{Y}}(\tilde{f}, \tilde{\varphi})\left|d \nu_{\tilde{x}}-d \nu_{\tilde{x}^{\prime}}\right| \leqslant 2 \varepsilon\left\|\nu_{\tilde{x}}-\nu_{\tilde{x}^{\prime}}\right\| \\
& =4 \varepsilon \sum_{v \in K^{0}}\left|\xi(v)-\xi^{\prime}(v)\right| \leqslant 4 \varepsilon \sqrt{\# K^{0}} d\left(\xi, \xi^{\prime}\right) \leqslant C d_{\widetilde{X}}\left(\tilde{x}, \tilde{x}^{\prime}\right) .
\end{aligned}
$$

Here $\left\|\nu_{\tilde{x}}-\nu_{\tilde{x}^{\prime}}\right\|$ denotes total variation. In the third inequality one applies (2.1) and Cauchy's inequality. Finally, $C=4 \varepsilon c \sqrt{\# K^{0}}$ with $c$ denoting the bi-Lipschitz constant of $\theta:|K| \rightarrow X$, and $\# K^{0}$ the number of vertices in $K$.

The above requirement $d_{\widetilde{X}}\left(\tilde{x}, \tilde{x}^{\prime}\right) \leqslant \delta$ can clearly be omitted because $\left(\widetilde{X}, d_{\tilde{X}}\right)$ is a geodesic space. Consider again the geodesic $\tilde{\varrho}$ joining $\tilde{x}$ with $\tilde{x}^{\prime}$, hence of length $d_{\widetilde{X}}\left(\tilde{x}, \tilde{x}^{\prime}\right)$. With points $\tilde{x}=\tilde{x}_{0}, \tilde{x}_{1}, \ldots, \tilde{x}_{k}=\tilde{x}^{\prime}$ of $\tilde{\varrho}$ such that $d_{\widetilde{X}}\left(\tilde{x}_{i-1}, \tilde{x}_{i}\right) \leqslant \delta$ for $i \in\{1, \ldots k\}$, we have from $(2.12)$

$$
\begin{aligned}
d_{\widetilde{Y}}\left(\tilde{f}(\tilde{x}), \tilde{f}\left(\tilde{x}^{\prime}\right)\right) & \leqslant \sum_{i=1}^{k} d_{\widetilde{Y}}\left(\tilde{f}\left(\tilde{x}_{i-1}\right), \tilde{f}\left(\tilde{x}_{i}\right)\right) \leqslant C \sum_{i=1}^{k} d_{\widetilde{X}}\left(\tilde{x}_{i-1}, \tilde{x}_{i}\right) \\
& =C d_{\widetilde{X}}\left(\tilde{x}, \tilde{x}^{\prime}\right) .
\end{aligned}
$$

The (Lipschitz) continuous map $\tilde{f}: \widetilde{X} \rightarrow \widetilde{Y}$ is $\varphi_{*}$-equivariant in the sense that

$$
\tilde{f} \circ \gamma=\varphi_{*}(\gamma) \circ \tilde{f} \quad \text { for } \gamma \in \Gamma_{X}
$$

Indeed, for $\tilde{x} \in \tilde{X}$, the measure $\nu_{\gamma(\tilde{x})}=\sum_{v \in K^{0}} \xi(v) \varepsilon_{\gamma(\tilde{\theta}(v))}$, (cf. (2.10)) is the image of $\nu_{\tilde{x}}$ under the isometry $\gamma$ of $\widetilde{X}$. We therefore obtain, by (2.4),

$$
\begin{gathered}
\int_{\widetilde{X}} d_{\widetilde{Y}}^{2}\left(\tilde{\varphi}\left(\tilde{x}^{\prime}\right), \tilde{y}\right) d \nu_{\gamma(\tilde{x})}\left(\tilde{x}^{\prime}\right)=\int_{\widetilde{X}} d_{\widetilde{Y}}^{2}\left((\tilde{\varphi} \circ \gamma)\left(\tilde{x}^{\prime}\right), \tilde{y}\right) d \nu_{\tilde{x}}\left(\tilde{x}^{\prime}\right) \\
=\int_{\widetilde{X}} d_{\widetilde{Y}}^{2}\left(\left(\varphi_{*}(\gamma) \circ \tilde{\varphi}\right)\left(\tilde{x}^{\prime}\right), \tilde{y}\right) d \nu_{\tilde{x}}\left(\tilde{x}^{\prime}\right)=\int_{\widetilde{X}} d_{\widetilde{Y}}^{2}\left(\tilde{\varphi}\left(\tilde{x}^{\prime}\right),\left(\varphi_{*}(\gamma)\right)^{-1}(\tilde{y})\right) d \nu_{\tilde{x}}\left(\tilde{x}^{\prime}\right),
\end{gathered}
$$

which is minimized (uniquely) as a function of $\tilde{y} \in \tilde{Y}$ both by $\tilde{f}(\gamma(\tilde{x}))$ and by $\left(\varphi_{*}(\gamma)\right)^{-1}(\tilde{y})=\tilde{f}(\tilde{x})$, that is, by $\tilde{y}=\left(\varphi_{*}(\gamma)\right)(\tilde{f}(\tilde{x}))$, so $(2.13)$ indeed holds at any point $\tilde{x} \in \widetilde{X}$.

Being thus $\varphi_{*}$-equivariant, the continuous map $\tilde{f}: \tilde{X} \rightarrow \widetilde{Y}$ has a continuous projection $f: X \rightarrow Y$, characterized by $\tilde{f}$ being a lift of $f$, that is, 
$f \circ p_{X}=p_{Y} \circ \tilde{f}$; cf. (2.3) and the text following (3.2) below. This projection is of class $\operatorname{Lip}(X, Y)$ because

$$
d_{Y}\left(f(x), f\left(x^{\prime}\right)\right) \leqslant d_{\widetilde{Y}}\left(\tilde{f}(\tilde{x}), \tilde{f}\left(\tilde{x}^{\prime}\right)\right) \leqslant C d_{\widetilde{X}}\left(\tilde{x}, \tilde{x}^{\prime}\right)=C d_{X}\left(x, x^{\prime}\right)
$$

for points $x, x^{\prime} \in X$ with $d_{X}\left(x, x^{\prime}\right) \leqslant \delta\left(\leqslant r_{X}\right)$, noting that $x, x^{\prime}$ are the projections of points $\tilde{x}, \tilde{x}^{\prime} \in \widetilde{X}$ with $d_{\widetilde{X}}\left(\tilde{x}, \tilde{x}^{\prime}\right)=d_{X}\left(x, x^{\prime}\right)$; cf. (2.2). As in the above case of $\tilde{f}$, the requirement $d_{X}\left(x, x^{\prime}\right) \leqslant \delta$ can be omitted, $\left(X, d_{X}\right)$ being a geodesic space. From the uniform convergence $\tilde{f}^{\varepsilon} \rightarrow \tilde{\varphi}$ follows by projection that the map $f^{\varepsilon} \in \operatorname{Lip}(X, Y)$ converges uniformly to $\varphi$ as $\varepsilon \rightarrow 0$.

(b) As noted after $(2.11)$, now with $\varepsilon=1 / n(n=1,2, \ldots)$, we have constructed a sequence of maps $\tilde{f}^{\varepsilon}$, now written $\tilde{f}_{1 / n}$, which are $\varphi_{*}$-equivariant and of class $\operatorname{Lip}(\tilde{X}, \tilde{Y})$ and converge uniformly to $\tilde{\varphi}$ as $n \rightarrow \infty$. For $t \in$ $\left[\frac{1}{n+1}, \frac{1}{n}\right]$ write $t=\frac{\tau}{n}+\frac{1-\tau}{n+1}$ with $\tau \in[0,1]$; and consider for each $\tilde{x} \in \widetilde{X}$ the geodesic $\left[\frac{1}{n+1}, \frac{1}{n}\right] \ni t \mapsto \tilde{f}_{t}(\tilde{x}) \in \widetilde{Y}$ given by

$$
\tilde{f}_{t}(\tilde{x})=\tau \tilde{f}_{1 / n}(\tilde{x})+(1-\tau) \tilde{f}_{1 /(n+1)}(\tilde{x})
$$

with the usual abuse of notation for "convex combinations" of points of $\widetilde{Y}$. For given $r>0$ there is by (2.11) an integer $N>0$ such that $\tilde{f}_{1 / n}(\tilde{x})$ lies in the convex ball $B_{\widetilde{Y}}(\tilde{\varphi}(\tilde{x}), r)$ for $n \geqslant N$ and $\tilde{x} \in \tilde{X}$. So does therefore $\tilde{f}_{t}(\tilde{x})$ for $t \in\left[\frac{1}{n+1}, \frac{1}{n}\right]$, hence for any $\left.\left.t \in\right] 0,1\right]$, and even for $t \in[0,1]$ when we define $\tilde{f}_{0}=\tilde{\varphi}$ and $\tilde{f}_{t}=\tilde{f}_{1 / N}$ for $\left.\left.t \in\right] 1 / N, 1\right]$ :

$$
d_{\widetilde{Y}}\left(f_{t}(\tilde{x}), \varphi(\tilde{x})\right) \leqslant r \quad \text { for } \tilde{x} \in \tilde{X}, t \in[0,1] .
$$

The metric $d_{\widetilde{Y}}$ being convex (cf. e.g. [2, Proposition 2.2]), it follows by (2.12) that each $\left.\left.\tilde{f}_{t}, t \in\right] 0,1\right]$, is of $\operatorname{class} \operatorname{Lip}(\widetilde{X}, \widetilde{Y})$. In terms of a common Lipschitz constant $c_{n}$ for $\tilde{f}_{1 / n}$ and $\tilde{f}_{1 /(n+1)}$ we have, in fact, for $\tilde{x}, \tilde{x}^{\prime} \in \tilde{X}$ and $t \in\left[\frac{1}{n+1}, \frac{1}{n}\right]$ in view of $(2.14)$ :

$$
\begin{aligned}
d_{\widetilde{Y}}\left(\tilde{f}_{t}(\tilde{x}), \tilde{f}_{t}\left(\tilde{x}^{\prime}\right)\right) \leqslant & \tau d_{\widetilde{Y}}\left(\tilde{f}_{1 / n}(\tilde{x}), \tilde{f}_{1 / n}\left(\tilde{x}^{\prime}\right)\right) \\
& +(1-\tau) d_{\widetilde{Y}}\left(\tilde{f}_{1 /(n+1)}(\tilde{x}), \tilde{f}_{1 /(n+1)}\left(\tilde{x}^{\prime}\right)\right) \\
\leqslant & \tau c_{n} d_{\widetilde{X}}\left(\tilde{x}, \tilde{x}^{\prime}\right)+(1-\tau) c_{n} d_{\widetilde{X}}\left(\tilde{x}, \tilde{x}^{\prime}\right)=c_{n} d_{\widetilde{X}}\left(\tilde{x}, \tilde{x}^{\prime}\right) .
\end{aligned}
$$

And from $\tilde{f}_{t} \in \operatorname{Lip}(\widetilde{X}, \widetilde{Y})$ for each $\left.\left.t \in\right] 0,1\right]$ follows $f_{t} \in \operatorname{Lip}(X, Y)$ for each such $t$ by the argument used above to show that $\tilde{f}=\tilde{f}^{\varepsilon} \in \operatorname{Lip}(\tilde{X}, \tilde{Y})$ implies $f \in \operatorname{Lip}(X, Y)$. 
Because geodesics in $\widetilde{Y}$ vary continuously with their endpoints (see $\left[2\right.$, p. 160]) it follows from $(2.14)$ and $(2.16)$ that $\tilde{f}(\tilde{x}, t):=\tilde{f}_{t}(\tilde{x})$ depends continuously on $(\tilde{x}, t) \in \widetilde{X} \times\left[\frac{1}{n+1}, \frac{1}{n}\right]$, hence altogether on $\left.\left.\widetilde{X} \times\right] 0,1\right]$, and even on $\widetilde{X} \times[0,1]$ by $(2.15)$ together with the continuity of $\underset{\tilde{f}}{\tilde{X}}(\cdot, 0)=\tilde{f}_{0}=\tilde{\varphi}$ on $\widetilde{X}$. The metric $d_{\widetilde{Y}}$ being convex, the continuous map $\tilde{f}_{t}$ is $\varphi_{*}$-equivariant for each $t \in[0,1]$; that is, (2.13) remains valid with $\tilde{f}$ replaced by $\tilde{f}_{t}$. Hence $\tilde{f}_{t}: \widetilde{X} \rightarrow \widetilde{Y}$ admits a continuous projection $f_{t}: X \rightarrow Y:$

$$
f_{t}(x):=p_{Y}\left(\tilde{f}_{t}(\tilde{x})\right), \quad \tilde{x} \in p_{X}^{-1}(x), \quad x \in X .
$$

Moreover, the map $f: X \times[0,1] \rightarrow Y$ given by $f(x, t)=f_{t}(x)$ is continuous in view of (2.17) because

$$
d_{Y}\left(f(x, t), f\left(x^{\prime}, t^{\prime}\right)\right) \leqslant d_{\widetilde{Y}}\left(f(\tilde{x}, t), f\left(\tilde{x}^{\prime}, t^{\prime}\right)\right) \rightarrow 0 \quad \text { as }\left(x^{\prime}, t^{\prime}\right) \rightarrow(x, t)
$$

and hence $\left(\tilde{x}^{\prime}, t^{\prime}\right) \rightarrow(\tilde{x}, t), p_{Y}$ being locally isometric. This completes the proof of Proposition 2.2.

Energy of maps. The concept of energy of maps will be used in the sequel also for maps from suitable locally compact spaces, in particular for maps between the universal covers of the above compact spaces $X$ and $Y$. In the rest of the present section we accordingly allow $(X, g)$ to be any locally compact separable m-dimensional admissible Riemannian polyhedron with simplex-wise smooth Riemannian metric $g$ and corresponding intrinsic Riemannian distance $d_{X}$ (as in $[9]$ ), and $\left(Y, d_{Y}\right.$ ) any complete metric space (in the absence of other indication). The definition and basic properties of energy of maps, due to [25] for maps from a Riemannian domain (in a Riemannian manifold), were extended in [9, Chapter 9] to the present polyhedral case. Denote by $L^{2}(X, Y)$, respectively $L_{\text {loc }}^{2}(X, Y)$, the space of all $\mu$-measurable maps $\varphi: X \rightarrow Y$ having separable essential range and for which $d_{Y}(\varphi(\cdot), q)$ is of class $L^{2}(X, \mu)$, respectively $L_{\text {loc }}^{2}(X, \mu)$, for some and hence any $q \in Y$. It is known for example that $L^{2}(X, Y)$ is a complete metric space with distance $D$ given by (cf. $[25$, p. 572])

$$
D^{2}\left(\varphi, \varphi^{\prime}\right)=\int_{X} d_{Y}^{2}\left(\varphi(x), \varphi^{\prime}(x)\right) d \mu(x) \quad \text { for } \varphi, \varphi^{\prime} \in L^{2}(X, Y) .
$$

For $\varphi \in L_{\text {loc }}^{2}(X, Y)$ the approximate energy density $e_{\varepsilon}(\varphi) \in L_{\text {loc }}^{1}(X, \mu)$, suitably normalized, is defined for $\varepsilon>0$ at every point $x \in X$ by

$$
e_{\varepsilon}(\varphi)(x)=\frac{1}{c_{m}} \int_{B_{X}(x, \varepsilon)} \frac{d_{Y}^{2}\left(\varphi(x), \varphi\left(x^{\prime}\right)\right)}{\varepsilon^{m+2}} d \mu\left(x^{\prime}\right)
$$


where $c_{m}=\omega_{m} /(m+2), \omega_{m}$ being the volume of the unit ball in $\mathbb{R}^{m}$; and $B_{X}(x, \varepsilon):=\left\{x^{\prime} \in X: d_{X}\left(x, x^{\prime}\right) \leqslant \varepsilon\right\}$. The energy of $\varphi \in L_{\mathrm{loc}}^{2}(X, Y)$ is defined by

$$
E(\varphi)=\sup _{f \in C_{c}(X,[0,1])}\left(\limsup _{\varepsilon \rightarrow 0} \int_{X} f e_{\varepsilon}(\varphi) d \mu\right) \quad(\leqslant \infty) .
$$

For any map $\varphi: X \rightarrow Y$ not of class $L_{\text {loc }}^{2}(X, Y)$ we put $E(\varphi)=\infty$. The class of all maps $\varphi: X \rightarrow Y$ of finite energy will be denoted here by $\mathcal{E}(X, Y)$ (instead of $W^{1,2}(X, Y)$ as in $[9,11-13,25,26]$, because we do not require that $\varphi \in L^{2}(X, Y)$ globally). For connected open subsets $U, V$ of $X$ we have by $(2.19)$,

$$
E(\varphi \mid U) \leqslant E(\varphi \mid V) \text { if } U \subset V
$$

because $\varphi \in L_{\text {loc }}^{2}(V, Y) \Rightarrow \varphi \mid U \in L_{\text {loc }}^{2}(U, Y)$ and because $B_{U}(x, \varepsilon) \subset B_{V}(x, \varepsilon)$ for any $x \in U$ and $\varepsilon>0$.

Every map $\varphi \in \mathcal{E}(X, Y)$ has quasicontinuous versions [12], to be used throughout, and any two of these are equal quasi-everywhere (q.e.), that is, everywhere except in some polar set [14, paragraph following Definition 1].

A map $\varphi: X \rightarrow Y$ is said to be locally of finite energy, and we write $\varphi \in \mathcal{E}_{\text {loc }}(X, Y)$, if it has one of the following three equivalent properties:

(i) $X$ can be covered by connected open sets $U \subset X$ such that $\varphi \mid U \in$ $\mathcal{E}(U, Y)$.

(ii) $\varphi \mid U \in \mathcal{E}(U, Y)$ for every connected open set $U \Subset X$.

(iii) $\varphi \in L_{\text {loc }}^{2}(X, Y)$, and there exists a function $e(\varphi) \in L_{\text {loc }}^{1}(X, \mu)$ such that

$$
\lim _{\varepsilon \rightarrow 0} \int_{X} f e_{\varepsilon}(\varphi) d \mu=\int_{X} f e(\varphi) d \mu, \quad f \in C_{c}(X) .
$$

In the affirmative case the unique weak limit $e(\varphi)$ from (iii) is termed the energy density of $\varphi$, and we have from (2.19)

$$
E(\varphi)=\int_{X} e(\varphi) d \mu \quad(\leqslant \infty)
$$

Clearly, $\varphi \mid U \in \mathcal{E}(U, Y)$ can be replaced by $\varphi \mid U \in \mathcal{E}_{\text {loc }}(U, Y)$ in (i) and (ii).

Slightly more generally, for any open set $A \subset X$, a map $\varphi: A \rightarrow Y$ is said to be locally of finite energy if the restriction of $\varphi$ to each component of $A$ is locally of finite energy; we then write $\varphi \in \mathcal{E}_{\text {loc }}(A, Y)$. (Recall that 
every connected open subset of $X$ is an admissible Riemannian polyhedron, like $X$ itself [18, Theorem 1 and Corollary 3].)

If $X$ can be covered by open sets $U \subset X$ such that $\varphi \mid U \in \mathcal{E}_{\text {loc }}(U, Y)$ (in analogy with (i) above) then clearly $\varphi \in \mathcal{E}_{\text {loc }}(X, Y)$.

Proof of equivalence of (i), (ii) and (iii). Clearly, (i) or (ii) implies that $\varphi \in L_{\text {loc }}^{2}(X, Y)$. Furthermore, (ii) $\Rightarrow$ (i) is obvious, $X$ being locally compact. Here, and in what follows, the topological interior of a subset $A$ of $X$ is denoted by $A^{\circ}$.

(i) $\Rightarrow$ (iii). Suppose that $\varphi$ satisfies (i). Then $\varphi$ has a quasicontinuous version because each $\varphi \mid U \in \mathcal{E}(U, Y)$ has such, and because quasicontinuity is a local property, $X$ being second countable. According to [42, Theorem 35 , p. 317] the given triangulation of $X$ can be refined so that every open star and hence every open $m$-simplex $s^{\circ}$ of $X$ becomes a subset of some set $U$ from the given open cover of $X$, and hence $E\left(\varphi \mid s^{\circ}\right) \leqslant E(\varphi \mid U)<\infty$ by (i), in view of $(2.20)$. From [9, Theorem $\left.9.1,(\mathrm{a}) \wedge(\mathrm{b}) \Rightarrow(\mathrm{a}) \wedge\left(\mathrm{b}^{\prime}\right) \Rightarrow(9.4)\right]$ we therefore deduce (iii). ${ }^{2}$

(iii) $\Rightarrow$ (ii). Clearly, $\varphi \mid U \in L_{\text {loc }}^{2}(U, Y)$ because $\varphi \in L_{\text {loc }}^{2}(X, Y)$. For $x \in U$ we have $B_{U}(x, \varepsilon) \subset B_{X}(x, \varepsilon)$, hence $e_{\varepsilon}(\varphi \mid U)(x) \leqslant e_{\varepsilon}(\varphi)(x)$. Fix $h \in C_{c}(X$, $[0,1])$ with $h=1$ in $U$. For any $f \in C_{c}(U,[0,1])$ we obtain

$$
\begin{aligned}
\int_{U} f e_{\varepsilon}(\varphi \mid U) d \mu & =\int_{U} f h e_{\varepsilon}(\varphi \mid U) d \mu \leqslant \int_{X} f h e_{\varepsilon}(\varphi) d \mu \\
& \rightarrow \int_{X} f h e(\varphi) d \mu \leqslant \int_{X} h e(\varphi) d \mu
\end{aligned}
$$

for $\varepsilon \rightarrow 0$. Taking supremum over all $f \in C_{c}(U,[0,1])$ we conclude by (2.19) that indeed $E(\varphi \mid U) \leqslant \int_{X} h e(\varphi) d \mu<\infty$.

Clearly $\mathcal{E}(X, Y) \subset \mathcal{E}_{\text {loc }}(X, Y)$. In the particular case $\left(Y, d_{Y}\right)=\mathbb{R}$ we have

$$
\mathcal{E}_{\mathrm{loc}}(X, \mathbb{R})=W_{\mathrm{loc}}^{1,2}(X), \quad E(u)=\int_{X}|\nabla u|^{2} d \mu \text { for } u \in W_{\mathrm{loc}}^{1,2}(X),
$$

see [25, Theorem 1.6.2] (for a Riemannian domain) and [9, Corollary 9.2].

As a consequence of [9, Lemma 4.4] (on volumes of balls), every locally Lipschitz map $\varphi: X \rightarrow Y$ is of class $\mathcal{E}_{\text {loc }}(X, Y)$.

\footnotetext{
${ }^{2}$ It may be noted that one may, in that proof, alternatively use simplexes instead of cubes, as in [13, from p. 771 (mid) to p. 772 (top)]. (We shall not use this fact in the present paper.)
} 
If $X$ is compact we have $\mathcal{E}(X, Y)=\mathcal{E}_{\text {loc }}(X, Y)$; and according to (2.19) the energy of an arbitrary map $\varphi \in L^{2}(X, Y)=L_{\mathrm{loc}}^{2}(X, Y)$ is then given simply by

$$
E(\varphi)=\limsup _{\varepsilon \rightarrow 0} \int_{X} e_{\varepsilon}(\varphi) d \mu \quad(\leqslant \infty)
$$

Lemma 2.3. (a) For any map $\varphi \in \mathcal{E}_{\mathrm{loc}}(X, Y)$ and any connected open set $A \subset X$ we have $\varphi \mid A \in \mathcal{E}_{\mathrm{loc}}(A, Y)$ and

$$
e(\varphi \mid A)=e(\varphi) \quad \mu \text {-a.e. in } A
$$

and hence $E(\varphi \mid A)=\int_{A} e(\varphi) d \mu$.

(b) For any two maps $\varphi, \psi \in \mathcal{E}_{\mathrm{loc}}(X, Y)$ and any $\mu$-measurable set $A \subset X$ such that $\mu\left(A \backslash A^{\circ}\right)=0$ we have the implications

$$
\begin{aligned}
\{\varphi=\psi \mu \text {-a.e. in } A\} & \Rightarrow\{e(\varphi)=e(\psi) \mu \text {-a.e. in } A\} \\
& \Rightarrow\left\{\int_{A} e(\varphi) d \mu=\int_{A} e(\psi) d \mu\right\}
\end{aligned}
$$

Proof. (a) Clearly, $B_{A}(x, \varepsilon) \subset B_{X}(x, \varepsilon)$ for $x \in A$, and equality prevails if $B_{X}(x, \varepsilon) \subset A$. Likewise, $\varphi \mid A \in \mathcal{E}_{\text {loc }}(A, Y)$ by (ii) preceding (2.21). For any $f \in C_{c}(A)$, any $x \in \operatorname{supp} f$, and any $0<\varepsilon<d_{X}(\operatorname{supp} f, X \backslash A)$ we therefore have $B_{A}(x, \varepsilon)=B_{X}(x, \varepsilon)$, and hence $e_{\varepsilon}(\varphi \mid A)(x)=e_{\varepsilon}(\varphi)(x)$. By $(2.21)$ (applied to $A$ in place of $X$ ) we infer that

$$
\int_{A} f e(\varphi \mid A) d \mu=\lim _{\varepsilon \rightarrow 0} \int_{A} f e_{\varepsilon}(\varphi \mid A) d \mu=\lim _{\varepsilon \rightarrow 0} \int_{X} f e_{\varepsilon}(\varphi) d \mu=\int_{X} f e(\varphi) d \mu
$$

which implies (2.25). It follows by (2.22) that $E(\varphi \mid A)=\int_{A} e(\varphi \mid A) d \mu=$ $\int_{A} e(\varphi) d \mu$.

(b) Suppose that $\varphi=\psi \mu$-a.e. in $A$, hence $\mu$-a.e. in the interior $A^{\circ}$ of $A$. It follows from (a), applied to each component $U$ of $A^{\circ}$, that $e(\varphi \mid U)=$ $e(\psi \mid U) \mu$-a.e. in $U$, and hence $\mu$-a.e. in $A^{\circ}$, and indeed in $A$ because $\mu\left(A \backslash A^{\circ}\right)=0$, by hypothesis.

Lemma 2.4. (a) Two quasicontinuous functions $X \rightarrow \mathbb{R}$ or maps $X \rightarrow Y$ which are equal $\mu$-a.e. in $X$ are equal quasi-everywhere (q.e.) in $X$.

(b) Every sequence of quasicontinuous functions $u_{n} \in W_{\mathrm{loc}}^{1,2}(X)$ converging to 0 in $W_{\mathrm{loc}}^{1,2}(X)$ has a subsequence converging to 0 pointwise q.e. in $X$. 
Proof. Part (a) for functions and Part (b) follow from [7, Théorèmes 5, 7], respectively, because $W^{1,2}(X)$ is a regular Dirichlet space with a kernel of positive type, according to [9, Proposition 5.1]. For two quasicontinuous maps $\varphi, \psi: X \rightarrow Y$ the quasicontinuous function $d_{Y}(\varphi(\cdot), \psi(\cdot))$ equals 0 $\mu$-a.e., hence q.e.

The following lemma will be applied in Step 1 of the proof of Theorem 4.1 below.

Lemma 2.5. For any E-bounded sequence of quasicontinuous maps $\varphi_{n} \in$ $\mathcal{E}(X, Y)$ converging in $L_{\text {loc }}^{2}(X, Y)$ to a map $\varphi$, we have $E(\varphi) \leqslant \liminf _{n} E\left(\varphi_{n}\right)$ $<\infty$, and

(a) for any $(m-1)$-simplex $\sigma$ of $X$,

$$
\operatorname{tr}_{\sigma} \varphi_{n} \rightarrow \operatorname{tr}_{\sigma} \varphi \text { in } L^{2}(\sigma) \text { as } n \rightarrow \infty \text {; }
$$

(b) If $X$ is compact there is a subsequence, still denoted $\left(\varphi_{n}\right)$, such that (with $\varphi$ likewise taken to be quasicontinuous)

$$
\frac{d_{Y}\left(\varphi_{1}, \varphi\right)+\ldots+d_{Y}\left(\varphi_{n}, \varphi\right)}{n} \rightarrow 0 \text { q.e. in } X \text { as } n \rightarrow \infty \text {. }
$$

For any $\varphi \in \mathcal{E}_{\text {loc }}(X, Y), \operatorname{tr}_{\sigma} \varphi$ denotes the trace of $\varphi$ on $\sigma$; it is of class $L^{2}(\sigma), \sigma$ being endowed with $(m-1)$-dimensional Hausdorff measure $\mathcal{H}_{m-1}$, $[25, \S 1.12],[9$, p. 153]. In terms of a triangulation $T=(K, \theta)$ of $X$ (see text preceding (2.1)) the measure $\mathcal{H}_{m-1}$ on $\sigma=\theta(|\tau|)$ is equivalent to Lebesgue measure on the geometric $(m-1)$-simplex $|\tau|$. For a quasicontinuous version of $\varphi, \operatorname{tr}_{\sigma} \varphi$ equals $\varphi \mathcal{H}_{m-1}$-a.e. on $\sigma$, see [13, p. 760].

Proof of Lemma 2.5. The stated lower semicontinuity of energy as well as property (a) are due to [25, Theorems 1.6.1 and 1.12.2] for a Riemannian domain $X$; for the present setting of an admissible Riemannian polyhedron, see $[9$, Lemma 9.1].

For the proof of (b), the quasicontinuous functions $u_{n}:=d_{Y}\left(\varphi_{n}, \varphi\right)$ are of class $\mathcal{E}(X, \mathbb{R}) \cap L^{2}(X, \mu)=W^{1,2}(X)$. By the triangle inequality we have

$$
\left|u_{n}(x)-u_{n}\left(x^{\prime}\right)\right| \leqslant d_{Y}\left(\varphi_{n}(x), \varphi_{n}\left(x^{\prime}\right)\right)+d_{Y}\left(\varphi(x), \varphi\left(x^{\prime}\right)\right),
$$

and hence by (2.19) and (2.23) together with $(a+b)^{2} \leqslant 2 a^{2}+2 b^{2}$

$$
\int_{X}\left|\nabla u_{n}\right|^{2} d \mu=E\left(u_{n}\right) \leqslant 2 E\left(\varphi_{n}\right)+2 E(\varphi) .
$$


Thus $\left(u_{n}\right)$ is a norm-bounded sequence in the Hilbert space $W^{1,2}(X)$. By passing to a subsequence, still denoted $\left(u_{n}\right)$, we achieve that $u_{n}$ converges weakly in $W^{1,2}(X)$ to some $u \in W^{1,2}(X)$. By the Banach-Saks theorem (cf. e.g. [32, p. 80]), we therefore have (after passing to a further subsequence $\left.\left(u_{n}\right)\right) \quad \sum_{i=1}^{n} u_{i} / n \rightarrow u$ in norm in $W^{1,2}(X)$, in particular in $L^{2}(X)$, and pointwise q.e. in $X$, by Lemma 2.4(b) applied to the sequence $u_{n}-u \rightarrow 0$ in $W^{1,2}(X)$, taking $u$ quasicontinuous. By hypothesis,

$$
\int_{X} u_{n}^{2} d \mu=\int_{X} d_{Y}^{2}\left(\varphi_{n}, \varphi\right) d \mu=D^{2}\left(\varphi_{n}, \varphi\right) \rightarrow 0 \quad \text { as } n \rightarrow \infty .
$$

It follows that $\left\|\sum_{i=1}^{n} u_{i} / n\right\|_{L^{2}(X)} \leqslant \sum_{i=1}^{n}\left\|u_{i}\right\|_{L^{2}(X)} / n \rightarrow 0$ because convergence implies Césaro summability. Thus $u=0 \mu$-a.e., and even q.e.; cf. Lemma 2.4(a). We conclude that indeed $\sum_{i=1}^{n} u_{i} / n \rightarrow u=0$ q.e. according to Lemma 2.4(b) after passing to yet a further subsequence.

Remark 2.6. Here are three possible definitions of harmonicity of a continuous map $\varphi \in \mathcal{E}_{\text {loc }}(X, Y)$ ( $X$ any locally compact separable admissible Riemannian polyhedron and $Y$ any complete metric space):

(a) $\varphi$ is locally E-minimizing, that is, the topology on $X$ has a base of connected open sets $U \Subset X$ for each of which $E(\psi \mid U) \geqslant E(\varphi \mid U)$ for every map $\psi \in \mathcal{E}_{\text {loc }}(X, Y)$ with $\psi=\varphi$ in $X \backslash U$ (this notion does not require continuity of $\varphi$ );

(b) the same requirement for continuous comparison maps $\psi \in \mathcal{E}_{\mathrm{loc}}(X, Y)$;

(c) $\varphi$ is bi-locally E-minimizing, that is: $X$ has a base of connected open sets $U \Subset X$ for each of which there is an open set $V \supset \varphi(U)$ in $Y$ such that $E(\psi \mid U) \geqslant E(\varphi \mid U)$ for every continuous map $\psi \in \mathcal{E}_{\mathrm{loc}}(X, Y)$ with $\psi(U) \subset V$ and $\psi=\varphi$ in $X \backslash U$.

Slightly more generally, for any open subset $A$ of $X$, a map $\varphi: A \rightarrow Y$ is said to have one of these three properties if the restriction of $\varphi$ to each component of $A$ has that property (recall that a connected open subset of $X$ has the properties required of $X$ ).

Of these three definitions of harmonicity, (a) is the one most commonly used; (b) is used for example in [25, §2.7] (the free homotopy problem); and (c) in [9, Chapter 12] (maps into Riemannian manifolds) and in Step 4 of the proof of Theorem 3.2 below. In each case (a), (b), or (c), one might equally well require that $U$ is an open star in a triangulation of $X$ fine enough that $U$ is the interior of its closure; in particular, $\mu(\partial U)=0$. Indeed, given a point $p \in X$ and a neighbourhood $N$ of $p$, consider any set $U$ as in (x) (where (x) 
stands for (a), (b), or (c)) such that $p \in U \subset N$. Choose open sets $U_{1}, U_{2}$ so that $p \in U_{1} \Subset U_{2} \Subset U$. By [42, Theorem 35], applied to the open cover of $X$ by $U_{2}$ and $X \backslash \bar{U}_{1}$, there exists a subdivision $T$ of the given triangulation of $X$ such that an open star $S \ni p$ satisfies $S \subset U_{2} \Subset U$. For the proof that (x) holds with $U$ replaced by $S$, consider any map $\psi \in \mathcal{E}_{\text {loc }}(X, Y)$ such that $\psi=\varphi$ in $X \backslash S$. [In case (b) or (c), take moreover $\psi$ continuous. In case (c), take an open set $V \subset Y$ so that $\varphi(U) \subset V$, and suppose that $\psi(S) \subset$ $V$; then $\varphi(S) \subset \varphi(U) \subset V$, and $\psi(U) \subset V$ because $\psi(U \backslash S)=\varphi(U \backslash S) \subset$ $V$.] It follows that $\psi=\varphi$ in $X \backslash U(\subset X \backslash S)$, and so $E(\psi \mid U) \geqslant E(\varphi \mid U)$. By Lemma 2.3, with $X, A$ replaced by $U, U \backslash S$, we conclude that indeed $E(\psi \mid S) \geqslant E(\varphi \mid S)$ because $\mu(\partial S)=0$ and because $e(\psi)=e(\varphi) \mu$-a.e. in $U \backslash S$.

If $X$ is noncompact then, by the latter argument, it would suffice in each definition (a), (b), or (c), to assume that the sets $U$ in question form a cover of $X$. (This would be false for compact $X$ since $X$ is covered by itself, and so property (a) would amount to $\varphi$ being globally E-minimizing. $)^{3}$

Each of the properties (a), (b), (c) is a local one. In the first place, if $\varphi: X \rightarrow Y$ has one of these properties, denoted (x), then so has $\varphi \mid A$ for every open set $A \subset X$ (we may assume that $A$ is connected). To see this, let $N$ be a neighbourhood of a point $p$ in $A$, and consider a connected open set $U \Subset A$ such that $p \in U \subset N$ and that (x) holds for $\varphi \in \mathcal{E}_{\text {loc }}(X, Y)$ and for that set $U$ [and moreover for some open set $V \supset \varphi(U)$ in case (c)]. Let $\psi \in \mathcal{E}_{\text {loc }}(A, Y)$ be as in (x) with $X$ replaced by $A$ (and $\varphi$ by $\varphi \mid A$ ), whilst keeping $U$ [and $V$ in case (c)]. Then $\psi$ extends to a well-defined map $\psi^{*} \in \mathcal{E}_{\text {loc }}(X, Y)$ such that $\psi^{*}=\varphi$ in $X \backslash A$ and hence in $X \backslash U$, in view of (i) preceding (2.21), applied to the open cover of $X$ by $X \backslash \bar{U}$ and $A$, noting that $\psi^{*}|X \backslash \bar{U}=\varphi| X \backslash \bar{U} \in \mathcal{E}_{\mathrm{loc}}(X \backslash \bar{U}, Y)$ and that $\psi^{*} \mid A=\psi \in \mathcal{E}_{\mathrm{loc}}(A, Y)$ [hence $\psi^{*}(U)=\psi(U) \subset V$ in case (c)]. By hypothesis, $E\left(\psi^{*} \mid U\right) \geqslant E(\varphi \mid U)$, that is $E(\psi \mid U) \geqslant E(\varphi \mid U)$ since $\psi^{*}=\psi$ in $U$. Thus $\varphi \mid A$ indeed has the property (x) in question.

Secondly, let $\left(A_{n}\right)$ be a sequence (or family) of open subsets of $X$, and write $A=\bigcup_{n} A_{n}$. For a map $\varphi: A \rightarrow Y$, if each $\varphi \mid A_{n}$ has property (x) then so has $\varphi$. For a given point $p \in A$ and neighbourhood $N$ of $p$ in $A$, fix $n$ with $p \in A_{n}$. By hypothesis, there exists an open set $U \Subset A_{n}$ with $p \in U \subset N$ [and moreover an open set $V \subset Y$ in case (c)] such that (x) holds with $X$ replaced by $A_{n}$ (and $\varphi$ by $\left.\varphi \mid A_{n}\right)$. Let $\psi \in \mathcal{E}_{\text {loc }}(A, Y)$ be as stated in (x)

\footnotetext{
${ }^{3}$ In [9, Definitions 10.1 and 12.1], and elsewhere concerning these definitions of harmonicity, it should thus have been required, as above, that the sets $U$ form a base for $X$ (not just a cover), or else that $X$ is noncompact.
} 
(now with $X$ replaced by $A$ ). In particular, $\psi=\varphi$ in $A \backslash U$ [and $\psi(U) \subset V$ in case (c)]. The map $\psi_{n}:=\psi \mid A_{n} \in \mathcal{E}_{\text {loc }}\left(A_{n}, Y\right)$ then satisfies $\psi_{n}=\varphi$ in $A_{n} \backslash U$ [and $\psi_{n}(U)=\psi(U) \subset V$ in case (c)]. It follows that $E\left(\psi_{n} \mid U\right) \geqslant E(\varphi \mid U)$, that is, $E(\psi \mid U) \geqslant E(\varphi \mid U)$, noting that $\psi_{n}=\psi$ in $U \Subset A_{n} \subset A$.

If $E(\varphi)<\infty$, the requirement $U \Subset X$ in (a), (b), or (c) (redundant when $X$ is compact) may equally well be replaced by $U \subset X$ and $\mu(\partial U)=0$ in each of the three definitions in view of Lemma 2.3 (because the relatively compact open sets $U$ from a base likewise constitute a base). Furthermore (again if $E(\varphi)<\infty)$, the inequality $E(\psi \mid U) \geqslant E(\varphi \mid U)$ becomes equivalent with $E(\psi) \geqslant E(\varphi)$ provided that $\mu(\partial U)=0$; this follows again from Lemma 2.3 because $\psi=\varphi \in X \backslash U$.

Lemma 2.7. Let $\left(Y, d_{Y}\right)$ be a complete geodesic space of nonpositive curvature. The three definitions of harmonicity (a), (b), and (c) in Remark 2.6 are equivalent for any continuous map $\varphi \in \mathcal{E}_{\text {loc }}(X, Y)$. The property of being a harmonic map from an open subset of $X$ to $Y$ is a local one.

Proof. Clearly, (a) $\Rightarrow(\mathrm{b}) \Rightarrow$ (c) (since we may take $V=Y$ in (c)), so it remains to prove that (c) $\Rightarrow(\mathrm{a})$. Consider first the case that $Y$ is simply connected. Suppose that $\varphi \in \mathcal{E}_{\text {loc }}(X, Y)$ is continuous and satisfies (c). For a given point $p \in X$ and a neighbourhood $N$ of $p$ let $U$ and $V$ be as in (c), with $p \in U \subset N$. As shown in Remark 2.6 we may assume that $U$ is an open star in a triangulation $T$ of $X$ and that $U$ is the interior of its closure in $X$. Because (a) is a local property (see again Remark 2.6) and because the open stars $U$ of $T$ cover $X$, it suffices to prove that the restriction $\varphi \mid U$ to each star $U$ has the property (a). Note that $U$ satisfies the Poincaré inequality (4.9) below, according to $[13$, Lemma $1(\mathrm{c})]$ applied to $X=\bar{U}$. After subdividing $T$ let $S \Subset U$ denote an open star in $U$ such that $p \in S$ and that $S$ is the interior of its closure $\bar{S}$. By [14, Theorem 1(a)] there exists a unique map $\varphi^{*} \in$ $\mathcal{E}(U, Y)$ which minimizes the energy among all maps $\psi$ in $\mathcal{E}(U, Y)$ such that $\psi=\varphi$ on $U \backslash S$. In particular, $\varphi^{*} \mid S$ is locally E-minimizing. Indeed, after a still further subdivision, let $s$ denote an open star in $S$ such that $s$ is the interior of its closure $\bar{s}$ in $S$. Consider any map $\sigma \in \mathcal{E}_{\text {loc }}(S, Y)$ such that $\sigma=$ $\varphi^{*}$ on $S \backslash s$. The extension $\sigma^{*}$ of $\sigma$ to $U$ defined by $\sigma^{*}=\varphi^{*}$ on $U \backslash S$, and hence on $U \backslash s$, is of class $\mathcal{E}_{\text {loc }}(U, Y)\left(\operatorname{since} \varphi^{*} \in \mathcal{E}(U, Y)\right.$ and $\left.\sigma \in \mathcal{E}_{\text {loc }}(S, Y)\right)$. Because $\sigma^{*}=\varphi^{*}=\varphi$ on $U \backslash S$ we conclude from the definition of $\varphi^{*}$ that $E\left(\sigma^{*}\right) \geqslant E\left(\varphi^{*}\right)$, and so indeed $E(\sigma) \geqslant E(\varphi)$ by Lemma $2.3(\mathrm{~b})$, noting that $\sigma^{*}=\varphi^{*}$ on $U \backslash S$ and that $\mu(\partial s)=0$. The locally $E$-minimizing map $\varphi^{*}$ has a (Hölder) continuous version, by [9, Theorem 10.1]. The star $S$ is regular, 
[9, Proposition 7.1]. By [14, Theorem 1(b)], $\varphi^{*}$ therefore is continuous on $\bar{S}$, and hence in all of $U$ because $\varphi^{*}|U \backslash S=\varphi| U \backslash S$ is continuous.

For the proof that $\varphi \mid U$ has property (a) (locally $E$-minimizing) consider, as above, any map $\psi \in \mathcal{E}_{\text {loc }}(U, Y)$ such that $\psi=\varphi$ in $U \backslash S$. Being simply connected, $Y$ is a $\operatorname{CAT}(0)$-space, by the Cartan-Hadamard theorem, cf. [2, p. 193f]. For $t \in[0,1]$ define on $U$ the map $\varphi_{t}=(1-t) \varphi+t \varphi^{*}$ into $Y$ (with the usual abuse of notation), and note that $\varphi_{t}=\varphi^{*}=\varphi$ on $U \backslash S$. Because $\varphi(\bar{S})$ and $\varphi^{*}(\bar{S})$ are compact and that geodesics in $Y$ vary continuously with their endpoints $([2$, p. 160]), it follows from $\varphi(\bar{S}) \subset \varphi(U) \subset V$ that $\varphi_{t}(\bar{S}) \subset V$ for small $t$. However, $\varphi_{t}(U \backslash S)=\varphi(U \backslash S) \subset \varphi(U) \subset V$, and so altogether $\varphi_{t}(U) \subset V$. Since $\varphi \mid U$ has property (c) it follows that

$$
E(\varphi \mid U) \leqslant E\left(\varphi_{t}\right) \leqslant(1-t) E(\varphi \mid U)+t E\left(\varphi^{*}\right)
$$

by (c) and by the energy convexity inequality [9, Equation (11.2)]. We conclude that $E(\varphi \mid U) \leqslant E\left(\varphi^{*}\right) \leqslant E(\psi)$, and so $\varphi \mid U$ indeed has property (a) from Remark 2.6 on each $U$ considered above, and therefore on all of $X .{ }^{4}$

If $Y$ is not simply connected, pass to the universal covers $\widetilde{X}, \widetilde{Y}$ of $X, Y$, and note that $\widetilde{X}$ can be covered by open subsets $\widetilde{A}$ projected isometrically onto open subsets $p_{X}(\widetilde{A})=A$ of $X$ covering $X$. If $\varphi: X \rightarrow Y$ satisfies (c) then so does each $\varphi \mid A$ (see Remark 2.6 above), hence also $\tilde{\varphi} \mid \widetilde{A}$, by isometry, letting the set $p_{Y}^{-1}(V) \subset \widetilde{Y}$ play the role of $V$. It follows (again by Remark 2.6) that the map $\tilde{\varphi}: \widetilde{X} \rightarrow \widetilde{Y}$ satisfies (c). Because $\widetilde{Y}$ is simply connected, $\tilde{\varphi}$ satisfies (a) as well, and so does each $\tilde{\varphi} \mid \widetilde{A}$, hence each $\varphi \mid A$, by isometry; and consequently $\varphi: X \rightarrow Y$ indeed has property (a). The latter assertion of Lemma 2.7 holds in view of Remark 2.6.

Remark 2.8. As mentioned in [9, Remark 12.1] the implication (c) $\Rightarrow$ (a) in Remark 2.6 likewise holds when $\left(Y, d_{Y}\right)$ is a complete geodesic space of curvature $\leqslant \kappa$ for some constant $\kappa>0$, provided that the image $\varphi(X)$ is a subset of some closed convex ball $B=B_{Y}(o, R)$ in $Y$ of radius $R<\pi /(2 \sqrt{\kappa})$

\footnotetext{
${ }^{4}$ In the justification for Lemma 2.7 with $Y$ simply connected (proposed in $[9$, Lemma 2.7]), the reference to [9, Theorem 11.3] was insufficient; it should be replaced as above by [13, Theorem 1], which includes continuity of the Dirichlet solution up to the boundary (but that result was not available at the time). This error has no consequences because the lemma was not used in the proof of [13, Theorem 1], nor in earlier work $[9,11,12]$. Moreover, at the quoted place in $[9]$, " $\varphi^{*}(S)$ for small $t$ " should read " $\varphi_{t}(S)$ for small $t$ " (as above).
} 
and such that $B$ is a CAT $(\kappa)$-space. The above proof of Lemma 2.7 carries over right away. ${ }^{5}$

Remark 2.9. In the setting of [19], if a harmonic map $\varphi: X \rightarrow Y(X$ a Riemannian manifold) is constant in some nonvoid open subset $\Omega$ of $X$, then $\varphi$ is constant in all of $X$ (assumed connected), see [19, p. 204]; this was shown there to imply that the order of the map is well defined in all of $\Omega$, which is essential for the theory developed in [19]. In the present setting, where $X$ is a polyhedron, the concept of order does not seem to be available. The stated uniqueness property is not relevant here, and actually fails in the simplest possible situation: Let $X$ be a tripod, obtained from the disjoint union of three copies $X_{1}, X_{2}, X_{3}$ of $[0,1]$ by identifying the three endpoints 0 , cf. [19, p. 178]. With $Y=\mathbb{R}$, take $\varphi\left|X_{1}=0, \varphi\right| X_{2}=$ id, and $\varphi \mid X_{3}=-$ id, where id denotes the identity map of $[0,1]$, cf. [9, Remark 5.3]. Then $\varphi: X \rightarrow \mathbb{R}$ is harmonic (see [9, Example 8.1]), and nonconstant, though constant on $X_{1}$, which has nonvoid interior.

\section{The free homotopy problem}

In the case of free homotopy there is no control of the growth of a minimizing sequence of equivariant maps; such a sequence may in fact converge to infinity in the locally compact space $\widetilde{Y},[25$, p. 647 and Remark 2.6.6].

The proof of Theorem 3.2 below is an adaptation of that of [25, Theorems 2.6.4 and 2.7.1] for manifold domains. With the present polyhedral domain $(X, g)$, harmonic maps generally are no longer Lipschitz continuous, but only Hölder continuous (Proposition 3.1 below); cf. [6], [9, Example 6.1]. The idea of Korevaar and Schoen is to modify a given minimizing sequence of equivariant maps into another which is moreover equicontinuous. The sequence of projected maps therefore subconverges uniformly to a map $\varphi$ which is shown to belong to the given homotopy class $\mathcal{H}$ and to minimize energy within $\mathcal{H}$. Lemma 2.7 above allows us to go beyond [25] by proving that, as expected from $[10,19]$, every energy minimizer relative to $\mathcal{H}$ is harmonic. And unlike [25], we do not assume that $b X=\varnothing$;

\footnotetext{
${ }^{5}$ In analogy with the preceding note the reference in $[9$, Remark 12.1] to $[9$, Theorem 11.4] shall now be replaced by [13, Theorem 3] (noting that the additional hypothesis there that either $Y$ be locally compact or $R<\pi /(4 \sqrt{\kappa})$ can be omitted according to [17, Theorem 2]).
} 
thus $X$ may be, more generally, any compact admissible Riemannian polyhedron (with or without boundary) with simplex-wise smooth Riemannian metric $g$.

In the following proposition, however, we relax the requirement that $X$ and $Y$ be compact (because the result will be applied to the universal covers $\widetilde{X}$ and $\widetilde{Y}$ ). See also Remark 3.3. For any compact set $X_{0} \subset X$ we denote by $\Lambda_{X_{0}}$ an ellipticity constant of $g$ restricted to $X_{0}[9, \mathrm{p} .48 \mathrm{f}]$. Recall from $[9,11]$ that every locally energy minimizing map has a (Hölder) continuous and hence harmonic version.

Proposition 3.1. Let $(X, g)$ be a locally compact admissible Riemannian polyhedron. For any compact set $X_{0} \subset X$ there exist constants $C, \alpha, \varrho>0$ depending only on $X, X_{0}, \Lambda_{X_{0}}$ such that, for any harmonic map $\varphi$ from $X$ to any complete simply connected geodesic space $\left(Y, d_{Y}\right)$ of nonpositive curvature,

$$
d_{Y}(\varphi(x), \varphi(y)) \leqslant C \sqrt{E(\varphi)} d_{X}^{\alpha}(x, y) \quad \text { for } x, y \in X_{0} \text { with } d_{X}(x, y) \leqslant \varrho .
$$

Proof. In view of [9, Chapters 4 and 5] our locally compact separable admissible Riemannian polyhedron $(X, g)$ satisfies the hypotheses of Biroli and Mosco $[3, \S 1]$. According to [3, Theorem 5.4] applied with $p=2$ to the function $d_{Y}(\varphi(\cdot), q), q \in Y$, which is weakly subharmonic by [9, Lemma 10.2(a)] (or see [17, Lemma 2]), we may choose $\varrho>0$, depending only on $X, X_{0}, \Lambda_{X_{0}}$, so that, by the triangle inequality for $\left(Y, d_{Y}\right)$, the oscillation $\omega\left(B_{X}(a, r)\right)$ of $\varphi$ over $B_{X}(a, r)$ for $a \in X_{0}$ and $0<r \leqslant \varrho$ satisfies the first two of the following three inequalities

$$
\begin{aligned}
\omega\left(B_{X}(a, r)\right) & \leqslant 2 \inf _{q \in Y} \sup _{B_{X}(a, r)} d_{Y}(\varphi(\cdot), q) \\
& \leqslant 2 c^{\prime} \inf _{q \in Y}\left(f_{B_{X}(a, 2 r)} d_{Y}^{2}(\varphi(\cdot), q) d \mu\right)^{1 / 2} \\
& \leqslant c^{\prime \prime} r^{1-m / 2} \sqrt{E(\varphi)}
\end{aligned}
$$

for constants $c^{\prime}, c^{\prime \prime}$ depending on $X, X_{0}, \Lambda_{X_{0}}$ only. The last inequality follows by application of [9, Lemma 4.4] (on ball volumes) and the weak Poincaré inequality [11, Corollary 1, p. 381] which clearly holds also for noncompact $X$ when $a \in X_{0} \Subset X$ (again with constants depending on $X, X_{0}, \Lambda_{X_{0}}$ ).

Choose $\varrho>0$ small enough that [11, Lemma 3 and Proposition 1(b)] hold with $\kappa=5$. These two results from [11] shall then replace, in [9, pp. 186-189], the repeated use of [9, Sublemma 10.1 and Lemma 10.1(a)], 
respectively. It follows by inspection of its proof that [9, Lemma 10.3] holds uniformly in the same sense for $a \in X_{0} \Subset X$. Local compactness of $\left(Y, d_{Y}\right)$ was only used towards the end of the proof of [9, Theorem 10.1] when a point $q$ was chosen (p. 191) in the intersection of the lower directed family of nonvoid compact essential images $\varphi\left(B_{X}^{e}(a, r)\right), r>0$. If $Y$ is not locally compact, choose instead any point $q_{j}=q_{j}(r)$ of $\varphi\left(B_{X}^{e}\left(a, \beta^{j} r\right)\right), j=1,2, \ldots$. Recall from $[9$, p. 190] that $\varepsilon, p$, and hence $\beta$, are constants $>0$. Take $j$ large enough so that $\beta^{j}<\varepsilon^{p}$. Then

$$
q_{j} \in \varphi\left(B_{X}^{e}\left(a, \beta^{j} r\right)\right) \subset \varphi\left(B_{X}^{e}\left(a, \varepsilon^{p} r\right)\right) .
$$

It follows that Equations (10.15) and (10.16) in [9] hold with $q$ replaced by $q_{j}$ (cf. the text following Equation (10.7), and Equations (10.13), (10.14) there). We then find that there are positive constants $R, A, \alpha$ depending only on $X, X_{0}, \Lambda_{X_{0}}$ such that, for any $r_{0} \leqslant R$ and $r \leqslant r_{0}$,

$$
\omega\left(B_{X}(a, r)\right) \leqslant A\left(r / r_{0}\right)^{\alpha} \omega\left(B_{X}\left(a, r_{0}\right)\right) .
$$

Taking $r_{0}=\varrho$ from the text preceding (3.1) we obtain for $x, y \in X_{0}$ with $a=y$ and $r:=d_{X}(x, y) \leqslant \varrho$,

$$
\begin{aligned}
d_{Y}(\varphi(x), \varphi(y)) & \leqslant \omega\left(B_{X}(y, r)\right) \leqslant A \varrho^{-\alpha} \omega\left(B_{X}(y, \varrho)\right) r^{\alpha} \\
& \leqslant A c^{\prime \prime} \varrho^{1-m / 2-\alpha} \sqrt{E(\varphi)} d_{X}^{\alpha}(x, y),
\end{aligned}
$$

which was to be proved.

Theorem 3.2. Let $(X, g)$ be a compact admissible Riemannian polyhedron, and let $\left(Y, d_{Y}\right)$ be a compact geodesic space of nonpositive curvature. Every (free) homotopy class $\mathcal{H}$ of continuous maps $X \rightarrow Y$ has an energy minimizer; and any such is harmonic and uniformly Hölder continuous. Two energy minimizers relative to $\mathcal{H}$ are identical if they agree at a point.

More generally (as expected from [21] in the classical setting of [9]): for any two energy minimizers $\varphi_{0}, \varphi_{1}$ relative to $\mathcal{H}$ there exists a homotopy $[0,1] \ni t \mapsto \varphi_{t} \in \mathcal{H}$ such that, for each $x \in X$, the path $[0,1] \ni t \mapsto \varphi_{t}(X) \in$ $Y$ is a constant-speed geodesic segment (not necessarily minimizing) of length $\tilde{d}$ independent of $x$. If $\tilde{d}<r_{Y}(\operatorname{cf}$. $(2.2))$ then $\tilde{d}=d_{Y}\left(\varphi_{0}(x), \varphi_{1}(x)\right)$, $x \in X$, and the geodesic segment $t \mapsto \varphi_{t}(x)$ becomes minimizing. And if $\varphi_{0}(x)=\varphi_{1}(x)$ for some $x \in X$ then $\tilde{d}=0$ and hence $\varphi_{0}=\varphi_{1}$.

Simple examples such as $X=Y=S^{1}$ show that there may be infinitely many minimizers in every homotopy class. In [25, Remark 2.6.6] the problem 
is posed as to when energy minimizers relative to $\mathcal{H}$ are unique up to domain or codomain isometries (as they are when $X=Y=S^{1}$ ).

Proof of Theorem 3.2. In view of Whitehead [42, Theorem 35, p. 317] or see $[37$, Theorem 14, p. 125] there is a subdivision $T$ of the given triangulation of $X$ such that every open star $S$ of $(X, T)$ is contained in some ball $B_{X}\left(x^{\prime}, \frac{1}{2} r_{X}\right)$ with $r_{X}$ as in (2.2). For any $x \in S$ we therefore have $x \in B_{X}\left(x^{\prime}, \frac{1}{2} r_{X}\right)$, and so $S \subset B_{X}\left(x^{\prime}, \frac{1}{2} r_{X}\right) \subset B_{X}\left(x, r_{X}\right)$. Accordingly, the connectivity components of $p_{X}^{-1}(S)$ have disjoint closures, each mapped isometrically onto $\bar{S}$ by $p_{X}$. By a further subdivision $T^{\prime}$ of $T$ we arrange that, for any $x \in X$, there exists a star $\bar{S}=S(x)$ of $(X, T)$ such that every star $S^{\prime}$ of $\left(X, T^{\prime}\right)$ containing $x$ has closure $\bar{S}^{\prime}$ contained in $S .{ }^{6}$ In particular, if two stars $S_{1}^{\prime}, S_{2}^{\prime}$ of $\left(X, T^{\prime}\right)$ have a point $x$ in common then $\bar{S}_{1}^{\prime} \cup \bar{S}_{2}^{\prime} \subset S(x)$.

Step 1. Construction of a modified sequence $\left(\tilde{\varphi}_{i}\right)$. Let $B^{j}, j \in\{1, \ldots, k\}$, denote the open stars of $\left(X, T^{\prime}\right)$. Here $k \geqslant 2$, for if $k=1$ we would have $B^{1}=X$, hence $X=$ a single vertex ( $X$ being compact), contradicting our hypothesis $\operatorname{dim} X \geqslant 1$ from the beginning of Section 2. Following [25, p. 649], choose a Lipschitz partition of unity $\left(\eta^{j}\right)_{j=1, \ldots, k}$ on $X$, subordinate to the cover $\left(B^{j}\right)$, cf. [9, p. $\left.41 \mathrm{f}\right]$; and for each $j \in\{1, \ldots, k\}$ two compact subsets $Z^{j}$ and $W^{j}$ of $X$ so that the support supp $\eta^{j}$ is contained in the interior of $Z^{j}$, that $Z^{j}$ is contained in the interior of $W^{j}$, and that $W^{j} \subset B^{j}$. In particular, $\bigcup_{j} Z^{j}=X$, and hence $\bigcup_{j} \widetilde{Z}^{j}=\widetilde{X}$ when defining $\widetilde{Z}_{j}=p_{X}^{-1}\left(Z_{j}\right)$. As in $\left[25\right.$, p. 649 (bottom)] (where, however, the $B^{j}$ are balls), consider the $\Gamma_{X}$-invariant pre-images $p_{X}^{-1}\left(B^{j}\right)$, and the $\Gamma_{X}$-invariant Lip functions $\tilde{\eta}^{j}=$ $\eta^{j} \circ p_{X}$ on $\widetilde{X}$. Clearly $\sum_{1}^{k} \tilde{\eta}^{j}=1$.

Fix a Lipschitz map $\chi \in \mathcal{H}$ (see Proposition 2.2(b), which replaces [25, Proposition 2.6.1]), a corresponding homomorphism $\chi_{*}: \Gamma_{X} \rightarrow \Gamma_{Y}$ (see Proposition 2.1), a base point $x_{0} \in X$, and a point $\tilde{x}_{0} \in p_{X}^{-1}\left(x_{0}\right)$. For any map $\varphi \in \mathcal{H}$, a $\chi_{*}$-equivariant lift $\tilde{\varphi}: \widetilde{X} \rightarrow \widetilde{Y}$ of $\varphi$ is obtained by choosing $\tilde{\varphi}\left(\tilde{x}_{0}\right) \in$ $p_{Y}^{-1}\left(\varphi\left(x_{0}\right)\right)$ (see $(2.3)$ and the end of the paragraph following Proposition 2.1) so that $\varphi_{*}=\chi_{*}$ and hence

$$
\tilde{\varphi} \circ \gamma=\chi_{*}(\gamma) \circ \tilde{\varphi} \quad \text { for } \gamma \in \Gamma_{X} .
$$

\footnotetext{
${ }^{6}$ Every point $x \in X$ lies in some star $S(x)$ of $(X, T)$, and $S(x)$ contains a ball $B_{X}\left(x, r_{x}\right)$. Finitely many of these balls cover $X$, and we define $r=$ the least among these finitely many $r_{x}$. Again by [42], choose the subdivision $T^{\prime}$ of $T$ so that every star $S^{\prime}$ of $\left(X, T^{\prime}\right)$ is a subset of some ball $B_{X}\left(x^{\prime}, \frac{1}{2} r\right)$. For any $x \in X$ and any star $S^{\prime}$ of $\left(X, T^{\prime}\right)$ containing $x$ we have $\bar{S}^{\prime} \subset B_{X}\left(x^{\prime}, \frac{1}{2} r\right)$ for some $x^{\prime} \in X$. In particular, $x \in B_{X}\left(x^{\prime}, \frac{1}{2} r\right)$, and so indeed $\bar{S}^{\prime} \subset B_{X}(x, r) \subset S(x)$.
} 
Conversely, every $\chi_{*}$-equivariant map $\tilde{\varphi}$ has in view of (3.2) a projection $\varphi: X \rightarrow Y$ of class $\mathcal{H}$, defined by $(2.3)$, that is, $\varphi(x)=p_{Y}(\tilde{\varphi}(\tilde{x}))$ for some $\tilde{x} \in p_{X}^{-1}(x)$. This makes sense because any other point of $p_{X}^{-1}(x)$ has the form $\gamma(\tilde{x})$ for some $\gamma \in \Gamma_{X}$, and so $p_{Y}((\tilde{\varphi} \circ \gamma)(\tilde{x}))=p_{Y}\left(\left(\chi_{*}(\gamma) \circ \tilde{\varphi}\right)(\tilde{x})\right)=$ $p_{Y}(\tilde{\varphi}(\tilde{x}))$ as before, according to (3.2). The projection $\varphi$ is continuous because we may take $\tilde{x} \in p_{X}^{-1}(x)$ to be a locally defined, continuous function of $x \in X$. Furthermore, $\varphi \in \mathcal{H}$ by Proposition 2.1, or as shown directly by geodesic homotopy: For $\tilde{x} \in \widetilde{X}$ and $t \in[0,1]$ denote $\widetilde{\Phi}(\tilde{x}, t)=(1-t) \tilde{\varphi}(\tilde{x})+$ $t \tilde{\chi}(\tilde{x})$ (with the usual abuse of notation). Then $\widetilde{\Phi}(\tilde{x}, t)$ is continuous on $\widetilde{X} \times$ $[0,1]$ (see $[2$, p. 160]), and Equation $(3.2)$ holds with $\tilde{\varphi}$ replaced by $\widetilde{\Phi}(\cdot, t)$. The projection $(x, t) \mapsto \Phi(x, t):=p_{Y}(\widetilde{\Phi}(\tilde{x}, t))$ is therefore well defined and continuous as a function of $(x, t) \in X \times[0,1]$. Because $\Phi(x, 0)=\varphi(x)$ and $\Phi(x, 1)=\chi(x), \varphi$ is indeed homotopic with $\chi$, i.e., $\varphi \in \mathcal{H}$.

Let $s_{\nu}, \nu \in\{1, \ldots, n\}$, denote the $m$-simplexes of $\left(X, T^{\prime}\right)$, and let $\widetilde{F}$ be the union of corresponding $m$-simplexes $\tilde{s}_{\nu}$ of $\widetilde{X}$ having the projections $p_{X}\left(\tilde{s}_{\nu}\right)=s_{\nu}, \nu \in\{1, \ldots, n\}$, but otherwise selected arbitrarily. The compact (generally disconnected) set $\widetilde{F}$ is then a fundamental "domain" of $\Gamma_{X}$, up to a $\tilde{\mu}$-nullset.

Consider any $\chi_{*}$-equivariant map $\tilde{\varphi} \in \mathcal{E}_{\mathrm{loc}}(\tilde{X}, \tilde{Y})$ (one such map is $\tilde{\chi}$ ). The restriction $\tilde{\varphi} \mid \widetilde{F}$ has finite energy, understood as the sum of the (finite) energies of the restrictions of $\tilde{\varphi}$ to the $m$-simplexes $\tilde{s}_{\nu}$ of $\widetilde{F}$; see [9, Theorem 9.1(a)]. Equivalently, $E(\tilde{\varphi} \mid \widetilde{F})=\int_{\widetilde{F}} e(\tilde{\varphi}) d \tilde{\mu}$, cf. (2.22). The projection $\varphi$ : $X \rightarrow Y$ has finite energy, and

$$
E(\tilde{\varphi} \mid \widetilde{F})=E(\varphi), \quad e(\tilde{\varphi})\left|\widetilde{F}=e(\varphi) \circ p_{X}\right| \widetilde{F} .
$$

Indeed, $E\left(\tilde{\varphi} \mid \tilde{s}_{\nu}\right)=E\left(\varphi \mid s_{\nu}\right)<\infty$ because $\tilde{\varphi} \mid \tilde{s}_{\nu}=\left(\varphi \mid s_{\nu}\right) \circ\left(p_{X} \mid \tilde{s}_{\nu}\right)$, where $p_{X} \mid \tilde{s}_{\nu}$ is an isometry of $\tilde{s}_{\nu}$ on $s_{\nu}$, cf. the first lines of the present proof. By summation over $\nu$ we obtain the former equation of (3.3) by [9, Theorem $9.1(\mathrm{a}),(\mathrm{b})]$ because $E(\varphi)<\infty$ and because $E(\varphi)=\sum_{\nu} E\left(\varphi \mid s_{\nu}\right)$ by [9, Remark 9.1, p. 153]. The latter equation of (3.3) now follows from $e(\tilde{\varphi})\left|\tilde{s}_{\nu}=e(\varphi) \circ p_{X}\right| \tilde{s}_{\nu}$.

Conversely, any $\chi_{*}$-equivariant lift $\tilde{\varphi}$ of a finite-energy map $\varphi \in \mathcal{H}$ has locally finite energy since $E(\tilde{\varphi} \mid \tilde{s})=E(\varphi \mid s)<\infty$ for any $m$-simplex $\tilde{s}$ of $\tilde{X}$ (writing $s=p_{X}(\tilde{s})$ ), again because $p_{X} \mid \tilde{s}$ is an isometry. It follows that $\tilde{\varphi} \in$ $\mathcal{E}_{\text {loc }}(\widetilde{X}, \widetilde{Y})$ (again by $[9$, Theorem $9.1(\mathrm{a}),(\mathrm{b})]$ ), so the above applies. We may thus define

$$
\begin{aligned}
E_{0} & =\inf \left\{E(\varphi): \tilde{\varphi} \text { is } \chi_{*} \text {-equivariant and of class } \mathcal{E}_{\text {loc }}(\widetilde{X}, \widetilde{Y})\right\} \\
& =\inf \{E(\varphi): \varphi \in \mathcal{H}\} \quad(<\infty) .
\end{aligned}
$$


Consider a sequence $\left(\tilde{\psi}_{i}\right)$ of $\chi_{*}$-equivariant maps which is minimizing for (3.4) in the sense that the projections $\psi_{i}$ of $\tilde{\psi}_{i}$ satisfy the equation

$$
\lim _{i \rightarrow \infty} E\left(\psi_{i}\right)=E_{0}
$$

As in $[25$, p. 650$]$ we begin by constructing for each $i \in\{1,2, \ldots\}$ and $j \in\{1,2, \ldots, k\}$ a unique $\chi_{*}$-equivariant energy minimizer $\tilde{\varphi}_{i}^{j}$ among all $\chi_{*^{-}}$ equivariant maps which agree with $\tilde{\psi}_{i}$ on $\widetilde{X} \backslash p_{X}^{-1}\left(B^{j}\right)$; so $\tilde{\varphi}_{i}^{j}$ solves an ordinary Dirichlet problem. For the moment, fix $j \in\{1, \ldots, k\}$ and one of the connectivity components $\widetilde{B}^{j}$ of $p_{X}^{-1}\left(B^{j}\right)$; thus $\widetilde{B}^{j}$ is an open star in $\widetilde{X}$ contained in some ball in $\widetilde{X}$ of radius $\frac{1}{2} r_{X}$ (see the first lines of the proof). The closure $\widetilde{X}^{j}$ of $\widetilde{B}^{j}$ is a closed star and thus a compact admissible Riemannian subpolyhedron of $\widetilde{X}$ with nonvoid boundary $b \widetilde{X}^{j}=\widetilde{X}^{j} \backslash \widetilde{B}^{j}$.

According to [13, Theorem 1(a)] applied to the polyhedron $\widetilde{X}^{j}$ and the prescribed boundary map $\operatorname{tr}_{b \widetilde{X}^{j}} \tilde{\psi}_{i}$ (the trace of $\tilde{\psi}_{i}$ on $b \widetilde{X}^{j}$ ) there exists a unique map $\tilde{\theta}: \widetilde{X}^{j} \rightarrow \widetilde{Y}$ which minimizes the energy among all finite-energy maps $\widetilde{X}^{j} \rightarrow \widetilde{Y}$ having the same trace on $b \widetilde{X}^{j}$ as $\widetilde{\psi}_{i}$, and that minimizer $\tilde{\theta}$ is locally E-minimizing in $\widetilde{B}^{j}$. In the first place, $\widetilde{B}^{j}$ is noncompact and covered by the open stars $\widetilde{U}$ in a triangulation of $\widetilde{B}^{j}$. Every map $\tilde{\beta} \in \mathcal{E}_{\text {loc }}\left(\widetilde{B}^{j}, \widetilde{Y}\right)$ such that $\tilde{\beta}=\tilde{\theta}$ on $\widetilde{B}^{j} \backslash \widetilde{U}$ extends by $\tilde{\theta}\left|b \widetilde{X}^{j}=\tilde{\psi}_{i}\right| b \widetilde{X}^{j}$ to a map $\tilde{\xi} \in \mathcal{E}\left(\widetilde{X}^{j}, \widetilde{Y}\right)$, for if $\widetilde{C}$ denotes the closure of $\widetilde{U}$ then the restrictions $\tilde{\theta}[\widetilde{X} \backslash \widetilde{C}$ and $\tilde{\beta}$ of $\tilde{\xi}$ to the open sets $\widetilde{X} \widetilde{C}^{j} \backslash \widetilde{C}$ and $\widetilde{B}^{j}$, respectively, covering $\widetilde{X}^{j}$, have locally finite energy, and so property (i) preceding Equation (2.21) is fulfilled. Secondly, $E(\tilde{\beta})=E(\tilde{\xi}) \geqslant E(\tilde{\theta})$, and hence $E(\tilde{\beta} \mid \widetilde{U}) \geqslant E(\tilde{\theta} \mid \widetilde{U})$ by Lemma 2.3 because $\tilde{\mu}(\partial \tilde{U})=0$ and $\tilde{\beta}=\tilde{\theta}$ in $\widetilde{B}^{j} \backslash \widetilde{U}$. Having thus verified that $\tilde{\theta}$ is locally $E$ minimizing in $\widetilde{B}^{j}$, we infer from [9, Theorem 10.1] that $\tilde{\theta}$ is continuous in $\widetilde{B}^{j}$ (after being redefined on a $\tilde{\mu}$-nullset), and hence harmonic in $\widetilde{B}^{j}$; cf. Remark 2.6. Furthermore, $\tilde{\theta}$ is continuous on $\widetilde{X}^{j}$, again by $[13$, Theorem 1(a)], because $\tilde{\psi}_{i}$ is continuous, in particular on $b \tilde{X}^{j}$. of $\tilde{X}$ :

$$
\tilde{\varphi}_{i}^{j}= \begin{cases}\chi_{*}(\gamma) \circ \tilde{\theta} \circ \gamma^{-1} & \text { in } \gamma\left(\widetilde{B}^{j}\right) \text { for any } \gamma \in \Gamma_{X} \\ \tilde{\psi}_{i} & \text { elsewhere in } \widetilde{X}\end{cases}
$$

Thus $\tilde{\varphi}_{i}^{j}$ agrees with $\tilde{\psi}_{i}$ outside $p_{X}^{-1}\left(B^{j}\right)=\bigcup_{\gamma \in \Gamma_{X}} \gamma\left(\widetilde{B}^{j}\right)$. Clearly, $\tilde{\varphi}_{i}^{j}$ is continuous (even on $\gamma\left(b \widetilde{X}^{j}\right), \gamma \in \Gamma_{X}$ ), and each $\tilde{\varphi}_{i}^{j}$ is of class $\mathcal{E}_{\text {loc }}(\widetilde{X}, \widetilde{Y})$, like $\tilde{\psi}_{i}$. Furthermore, $\tilde{\varphi}_{i}^{j}: \widetilde{X} \rightarrow \widetilde{Y}$ is $\chi_{*}$-equivariant (cf. (3.2)) along with $\tilde{\psi}_{i}$; and $\tilde{\varphi}_{i}^{j}$ is Hölder continuous and harmonic in $\gamma\left(\widetilde{B}^{j}\right)$, along with $\tilde{\theta}$ and $\tilde{\theta} \circ \gamma^{-1}$. 
The projection $\varphi_{i}^{j} \in \mathcal{E}(X, Y)$ of $\tilde{\varphi}_{i}^{j} \in \mathcal{E}_{\mathrm{loc}}(\widetilde{X}, \widetilde{Y})$ agrees with $\psi_{i}$ outside $B^{j}=p_{X}\left(\widetilde{B}^{j}\right)=p_{X}\left(\gamma\left(\widetilde{B}^{j}\right)\right)$. The energy $E\left(\varphi_{i}^{j}\right)$ satisfies

$$
E_{0} \leqslant E\left(\varphi_{i}^{j}\right) \leqslant E\left(\psi_{i}\right)
$$

This is because $E(\tilde{\theta}) \leqslant E\left(\tilde{\psi}_{i} \mid \tilde{X}^{j}\right)$ by definition of $\tilde{\theta}$, and hence by (3.6) and $\gamma=\mathrm{id}$, followed by projection: $E\left(\varphi_{i}^{j} \mid X^{j}\right) \leqslant E\left(\psi_{i} \mid X^{j}\right)$, where $X^{j}:=$ $p_{X}\left(\widetilde{X}^{j}\right)$. Since $\mu\left(X^{j} \backslash B^{j}\right)=0$ it follows by Lemma $2.3\left(\right.$ a) that $E\left(\varphi_{i}^{j} \mid B^{j}\right) \leqslant$ $E\left(\psi_{i} \mid B^{j}\right)$. For each $j$ the sequence of $\chi_{*}$-equivariant maps $\tilde{\varphi}_{1}^{j}, \tilde{\varphi}_{2}^{j}, \ldots$ is therefore minimizing, along with the sequence $\left(\tilde{\psi}_{i}\right)$.

The desired modification $\tilde{\varphi}_{i}$ of $\tilde{\psi}_{i}$ is now defined (as in [25, p. 650]) by having for its value at $\tilde{x} \in \widetilde{X}$ the average of the $L^{2}$-maps $\tilde{\varphi}_{i}^{1}, \ldots, \tilde{\varphi}_{i}^{k}$ from (3.6) with respect to the probability measure $\nu_{\tilde{x}}$ on $\{1, \ldots, k\}$ given by

$$
\nu_{\tilde{x}}(\{j\})=\tilde{\eta}^{j}(\tilde{x}), \quad j \in\{1, \ldots, k\},
$$

with $\tilde{\eta}^{j}(\tilde{x})$ as defined in the first paragraph of the present Step 1. In terms of [25, Lemma 2.5.1] this means that, for each $\tilde{x} \in \widetilde{X}$,

$$
\tilde{\varphi}_{i}(\tilde{x}) \text { minimizes } \sum_{j=1}^{k} \tilde{\eta}^{j}(\tilde{x}) d_{\tilde{Y}}^{2}\left(\tilde{\varphi}_{i}^{j}(\tilde{x}), \tilde{y}\right) \text { as a function of } \tilde{y} \in \tilde{Y} \text {. }
$$

It follows that $\tilde{y}=\tilde{\varphi}_{i}(\gamma(\tilde{x}))$ minimizes

$$
\begin{aligned}
\sum_{j=1}^{k} \tilde{\eta}^{j}(\gamma(\tilde{x})) d_{\widetilde{Y}}^{2}\left(\tilde{\varphi}_{i}^{j}(\gamma(\tilde{x})), \tilde{y}\right) & =\sum_{j=1}^{k} \tilde{\eta}^{j}(\tilde{x}) d_{\widetilde{Y}}^{2}\left(\chi_{*}(\gamma)\left(\tilde{\varphi}_{i}^{j}(\tilde{x})\right), \tilde{y}\right) \\
& =\sum_{j=1}^{k} \tilde{\eta}^{j}(\tilde{x}) d_{\widetilde{Y}}^{2}\left(\tilde{\varphi}_{i}^{j}(\tilde{x}),\left(\chi_{*}(\gamma)\right)^{-1}(\tilde{y})\right),
\end{aligned}
$$

which is also minimized by $\left(\chi_{*}(\gamma)\right)^{-1}(\tilde{y})=\tilde{\varphi}_{i}(\tilde{x})$, that is, by $\tilde{y}=\left(\chi_{*}(\gamma)\right)\left(\tilde{\varphi}_{i}\right.$ $(\tilde{x}))$. Consequently, $\tilde{\varphi}_{i}(\gamma(\tilde{x}))=\left(\chi_{*}(\gamma)\right)\left(\tilde{\varphi}_{i}(\tilde{x})\right)$; so (3.2) holds (with $\tilde{\varphi}$ replaced by $\tilde{\varphi}_{i}$ ).

Step 2. The sequence of maps $\tilde{\varphi}_{i}: \widetilde{X} \rightarrow \widetilde{Y}$ is uniformly Hölder equicontinuous. Fix $\delta_{0}>0$ independently of $j$ so that $\delta_{0} \leqslant r_{X}$ from (2.2) and that

$$
\begin{array}{r}
\left\{x \in X: d_{X}\left(x, \operatorname{supp} \eta^{j}\right) \leqslant \delta_{0}\right\} \subset Z^{j}, \\
W^{j}:=\left\{x \in X: d_{X}\left(x, Z^{j}\right) \leqslant \delta_{0}\right\} \subset B^{j}
\end{array}
$$

for $j \in\{1, \ldots, k\}$; cf. the first paragraph of Step 1 . Then $Z^{j}$ and $W^{j}$ are compact subsets of $B^{j}$. Because $\widetilde{Y}$ is simply connected it follows by 
Proposition 3.1 with $X$ replaced by $\widetilde{B}^{j}$ that the harmonic maps $\tilde{\varphi}_{i}^{j}: \widetilde{X} \rightarrow \widetilde{Y}$ from (3.6) are uniformly Hölder equicontinuous on the compact set $\widetilde{B}^{j} \cap$ $p_{X}^{-1}\left(W^{j}\right)$ and even in $\widetilde{W}^{j}:=p_{X}^{-1}\left(W^{j}\right)=\bigcup_{\gamma \in \Gamma_{X}} \gamma\left(\widetilde{B}^{j} \cap p_{X}^{-1}\left(W^{j}\right)\right)$ because $\tilde{\varphi}_{i}^{j}$ is $\chi_{*}$-equivariant, that $\gamma$ and $\chi_{*}(\gamma)$ are isometries, and that two points $\tilde{x}, \tilde{y} \in$ $\widetilde{W}^{j}$ with $d_{\widetilde{X}}(\tilde{x}, \tilde{y}) \leqslant \varrho$ lie in the same set $\gamma\left(\widetilde{B}^{j} \cap p_{X}^{-1}\left(W^{j}\right)\right)$ when we take $\varrho \leqslant \delta_{0}$; cf. (2.2) and subsequent text. Consequently,

$$
d_{\widetilde{Y}}\left(\tilde{\varphi}_{i}^{j}(\tilde{x}), \tilde{\varphi}_{i}^{j}(\tilde{y})\right) \leqslant C \sqrt{E\left(\psi_{i}\right)} d_{\tilde{X}}^{\alpha}(\tilde{x}, \tilde{y}) \quad \text { for } \tilde{x}, \tilde{y} \in \widetilde{W}^{j}, d_{\tilde{X}}(\tilde{x}, \tilde{y}) \leqslant \varrho
$$

in view of (3.7) and (3.10), with $C, \alpha, \varrho$ depending on $X, \Lambda_{X}$ only. In particular, $C, \alpha, \varrho$ in (3.11) may be taken independently of $i \in\{1,2, \ldots\}$ and $j \in\{1, \ldots, k\}$. We may clearly take $\alpha \leqslant 1$. Note that (3.11) replaces in our setting the Lipschitz continuity established in [25, Theorem 2.6.4].

Continuing much as in $\left[25\right.$, p. 652 (mid)], consider points $\tilde{x} \in \widetilde{Z}^{j} \cap \widetilde{Z}^{l}$ $(j, l \in\{1, \ldots, k\})$ and $\tilde{y} \in \widetilde{X}$ with $d_{\tilde{X}}(\tilde{x}, \tilde{y}) \leqslant \delta$ for a suitable $\delta$ with $0<\delta \leqslant$ $\varrho \leqslant \delta_{0}$ ( $\delta$ to be determined in the paragraph containing (3.17) and (3.18) below). Then $\tilde{y} \in \widetilde{W}^{j} \cap \widetilde{W}^{l}$. By (3.11) and the triangle inequality we obtain

$$
d_{\widetilde{Y}}\left(\tilde{\varphi}_{i}^{j}(\tilde{y}), \tilde{\varphi}_{i}^{l}(\tilde{y})\right) \geqslant d_{\widetilde{Y}}\left(\tilde{\varphi}_{i}^{j}(\tilde{x}), \tilde{\varphi}_{i}^{l}(\tilde{x})\right)-2 C \sqrt{E\left(\psi_{i}\right)} \delta^{\alpha},
$$

and hence by integration with respect to $\tilde{y}$ (again for $\tilde{x} \in \widetilde{Z}^{j} \cap \widetilde{Z}^{l} \subset p_{X}^{-1}\left(B^{j} \cap\right.$ $\left.B^{l}\right)$ ):

$C_{1} \int_{B_{\tilde{X}}(\tilde{x}, \delta)} d_{\widetilde{Y}}^{2}\left(\tilde{\varphi}_{i}^{j}(\tilde{y}), \tilde{\varphi}_{i}^{l}(\tilde{y})\right) d \tilde{\mu}(\tilde{y}) \geqslant \delta^{m}\left(d_{\widetilde{Y}}\left(\tilde{\varphi}_{i}^{j}(\tilde{x}), \tilde{\varphi}_{i}^{l}(\tilde{x})\right)-2 C \sqrt{E\left(\psi_{i}\right)} \delta^{\alpha}\right)^{2}$

provided that $d_{\widetilde{Y}}\left(\tilde{\varphi}_{i}^{j}(\tilde{x}), \tilde{\varphi}_{i}^{l}(\tilde{x})\right) \geqslant 2 C \sqrt{E\left(\psi_{i}\right)} \delta^{\alpha}$. (We have applied [9, Lemma 4.4] about ball volumes.) This inequality (3.12) replaces [25, (2.6xvii)] in our setting.

Because $x:=p_{X}(\tilde{x}) \in B^{j} \cap B^{l}$, we have $B^{j} \cup B^{l} \Subset S$ for some star $S$ of $(X, T)$, cf. the beginning of the proof. Denote by $\widetilde{S}, \widetilde{B}^{j}, \widetilde{B}^{l}$ the component of $p_{X}^{-1}(S), p_{X}^{-1}\left(\widetilde{B}^{j}\right), p_{X}^{-1}\left(B^{l}\right)$, respectively, containing the given point $\tilde{x}$. This definition of $\widetilde{B}^{j}$ (and $\widetilde{B}^{l}$ ) agrees with the previous one (in the paragraph following (3.5)); and we have $\widetilde{B}^{j} \cup \widetilde{B}^{l} \Subset \widetilde{S}$. Choose a compact "fundamental domain" $\widetilde{F} \subset \widetilde{X}$ for $p_{X}$ relative to $T^{\prime}$ (cf. the paragraph preceding the one containing (3.3)) so that $\widetilde{F}$ contains $\widetilde{S}$; this is possible because $\widetilde{S}$ is contained in some ball in $\widetilde{X}$ of radius $r_{X}$, so no two simplexes of $\widetilde{S}$ are congruent modulo $\Gamma_{X}$; cf. text following $(2.2)$. The function $d_{\widetilde{Y}}\left(\tilde{\varphi}_{i}^{j}, \tilde{\varphi}_{i}^{l}\right)$ considered on $\widetilde{F}$ equals zero outside $\widetilde{B}^{j} \cup \widetilde{B}^{l} \Subset \widetilde{S}$ because $\tilde{\varphi}_{i}^{j}=\psi_{i}$ in $\widetilde{F} \backslash \widetilde{B}^{j}$ whereas $\tilde{\varphi}_{i}^{l}=\psi_{i}$ 
in $\widetilde{F} \backslash \widetilde{B}^{l}$. It follows that

$$
\int_{\widetilde{S}} d_{\widetilde{Y}}^{2}\left(\tilde{\varphi}_{i}^{j}, \tilde{\varphi}_{i}^{l}\right) d \tilde{\mu} \leqslant C_{2} \int_{\widetilde{S}}\left|\nabla d_{\widetilde{Y}}\left(\tilde{\varphi}_{i}^{j}, \tilde{\varphi}_{i}^{l}\right)\right|^{2} d \tilde{\mu}
$$

by the Poincaré inequality (4.9) below (for the present $\widetilde{S}$ ). Now apply the energy convexity inequality [25, (2.2vi); 9, (11.2)] (cf. Equation (3.33) below), with $t=\frac{1}{2}$ to the $\chi_{*}$-equivariant maps $\tilde{\varphi}_{i}^{j}, \quad \tilde{\varphi}_{i}^{l}$, and their $\chi_{*}$-equivariant midpoint map $\tilde{w}_{i}^{j l}$ with projection $w_{i}^{j l}$, all three maps $\widetilde{X} \rightarrow \widetilde{Y}$ being restricted to the $m$-simplexes $\tilde{s}_{\nu}$ of $\widetilde{F}$. By adding over $\nu=1, \ldots, n$ we obtain in view of (3.3), (3.4) and (3.7) the bound

$$
\int_{\widetilde{S}}\left|\nabla d_{\widetilde{Y}}\left(\tilde{\varphi}_{i}^{j}, \tilde{\varphi}_{i}^{l}\right)\right|^{2} d \tilde{\mu} \leqslant 4\left(E\left(\psi_{i}\right)-E\left(w_{i}^{j l}\right)\right)
$$

for the integral on the right-hand side of (3.13) because $\widetilde{S} \subset \widetilde{F}$ and $\tilde{\varphi}_{i}^{j}=\tilde{\varphi}_{i}^{l}$ in $\widetilde{F} \backslash \widetilde{S}$, hence $\nabla d_{\widetilde{Y}}\left(\tilde{\varphi}_{i}^{j}, \tilde{\varphi}_{i}^{l}\right)=0 \tilde{\mu}$-a.e. in $\widetilde{F} \backslash \widetilde{S}$ by $[9$, Remark 5.2]. We have $B_{\widetilde{X}}(\tilde{x}, \delta) \subset B_{\widetilde{X}}\left(\tilde{x}, \delta_{0}\right) \subset \widetilde{W}^{j} \cap \widetilde{W}^{l}$. By definition of $\widetilde{B}^{j}, \widetilde{B}^{l}$ it follows that $B_{\widetilde{X}}\left(\tilde{x}, \delta_{0}\right) \subset \widetilde{B}^{j} \cap \widetilde{B}^{l} \subset \widetilde{B}^{j} \cup \widetilde{B}^{l}$. We may therefore combine (3.12), (3.13), and (3.14), to get

$$
d_{i} \leqslant 2 C \sqrt{E\left(\psi_{i}\right)} \delta^{\alpha}+2 C_{1}^{1 / 2} C_{2}^{1 / 2} \delta^{-m / 2} \sqrt{E\left(\psi_{i}\right)-E\left(w_{i}^{j l}\right)}
$$

in terms of the notation

$$
d_{i}=\sup \left\{d_{\widetilde{Y}}\left(\tilde{\varphi}_{i}^{j}(\tilde{x}), \tilde{\varphi}_{i}^{l}(\tilde{x})\right): \tilde{x} \in \widetilde{Z}^{j} \cap \widetilde{Z}^{l} ; j, l \in\{1, \ldots, k\}\right\} .
$$

Note that the points $\tilde{x}$ where $d_{\widetilde{Y}}\left(\tilde{\varphi}_{i}^{j}(\tilde{x}), \tilde{\varphi}_{i}^{l}(\tilde{x})\right)<2 C \sqrt{E\left(\psi_{i}\right)} \delta^{\alpha}$, left out of consideration after (3.12), trivially do not violate (3.15). If $d_{i} \geqslant 4 C \sqrt{E\left(\psi_{i}\right)}$ $\varrho^{\alpha}$ take the above $\delta=\varrho$; then $\frac{1}{2} d_{i} \leqslant d_{i}-2 C \sqrt{E\left(\psi_{i}\right)} \varrho^{\alpha}$, and so by $(3.15)$

$$
d_{i} \leqslant C_{3} \sqrt{E\left(\psi_{i}\right)-E\left(w_{i}^{j l}\right)}
$$

with $C_{3}=4 C_{1}^{1 / 2} C_{2}^{1 / 2} \varrho^{-m / 2}$. If instead $d_{i}<4 C \sqrt{E\left(\psi_{i}\right)} \varrho^{\alpha}$, take $\delta^{\alpha}=d_{i} /(4 C$ $\left.\sqrt{E\left(\psi_{i}\right)}\right)\left(<\varrho^{\alpha}\right)$; this implies $d_{i}=2 d_{i}-4 C \sqrt{E\left(\psi_{i}\right)} \delta^{\alpha}$, and so by $(3.15)$,

$$
d_{i}^{m /(2 \alpha)+1}=d_{i}^{m /(2 \alpha)} d_{i}=\left(4 C \sqrt{E\left(\psi_{i}\right)} \delta^{\alpha}\right)^{m /(2 \alpha)}\left(2 d_{i}-4 C \sqrt{E\left(\psi_{i}\right)} \delta^{\alpha}\right)
$$

$$
\leqslant C_{4}{\sqrt{E\left(\psi_{i}\right)}}^{m /(2 \alpha)} \sqrt{E\left(\psi_{i}\right)-E\left(w_{i}^{j l}\right)}
$$


with $C_{4}=(4 C)^{m /(2 \alpha)} 4 C_{1}^{1 / 2} C_{2}^{1 / 2}$ (note that $\delta$ drops out here). In any case,

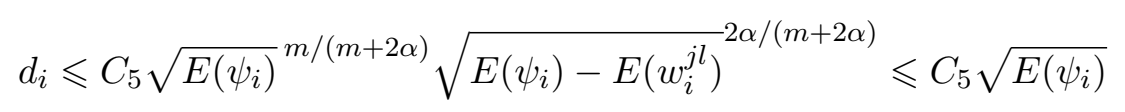

(with $C_{5}=\max \left\{C_{3}, C_{4}^{2 \alpha /(m+2 \alpha)}\right\}$ ), having inserted $E\left(\psi_{i}\right) \geqslant E\left(\psi_{i}\right)-E\left(w_{i}^{j l}\right)$ for the latter inequality. Since $\tilde{w}_{i}^{j l}$ is $\chi_{*}$-equivariant (along with $\tilde{\varphi}_{i}^{j}$ and $\tilde{\varphi}_{i}^{l}$ ) we have $E\left(w_{i}^{j l}\right) \geqslant E_{0}$, and since $\left(\tilde{\psi}_{i}\right)$ is minimizing we obtain from (3.19) $\lim _{i} d_{i}=0$, thus recovering $[25,(2.6 \mathrm{xv})]$ in our setting.

In view an application in Section 5 we continue without taking this and other minimization properties into account until later. We proceed to derive $[25,(2.6 x i v)]$ from (3.16). Recall from the first paragraph of Step 1 that $\operatorname{supp} \eta^{j} \subset Z^{j}$ and hence supp $\tilde{\eta}^{j} \subset \widetilde{Z}^{j}$. For each $\tilde{x} \in \widetilde{X}$ and $i \in\{1,2, \ldots\}$ we find from (3.9) that (with an apparently different notation)

$$
\tilde{\varphi}_{i}(\tilde{x}) \text { minimizes } \sum_{j \in J(\tilde{x})} \tilde{\eta}^{j}(\tilde{x}) d_{\tilde{Y}}^{2}\left(\tilde{\varphi}_{i}^{j}(\tilde{x}), \tilde{y}\right) \quad \text { for } \tilde{y} \in \tilde{Y}
$$

where $J(\tilde{x}):=\left\{j \in\{1, \ldots, k\}: \tilde{x} \in \widetilde{Z}^{j}\right\} \quad$ (note that $\tilde{\eta}^{j}(\tilde{x})=0$ for $\tilde{x} \notin \widetilde{Z}^{j}$ ). The points $\tilde{\varphi}_{i}^{j}(\tilde{x})$ with $j \in J(\tilde{x})$ form a set of diameter $\leqslant d_{i}$, by (3.16). These points $\tilde{\varphi}_{i}^{j}(\tilde{x})$ therefore lie in each ball $B_{\widetilde{Y}}\left(\tilde{\varphi}_{i}^{l}(\tilde{x}), d_{i}\right)$ for which $\tilde{x} \in \widetilde{Z}^{l}$. Every ball in $\widetilde{Y}$ being convex, it follows by [25, Proposition 2.5.4] (or see [9, Lemma 10.4]) that the average $\tilde{\varphi}_{i}(\tilde{x})$ from (3.20) likewise lies in $B_{\widetilde{Y}}\left(\tilde{\varphi}_{i}^{l}(\tilde{x}), d_{i}\right)$ when $\tilde{x} \in \widetilde{Z}^{l}$. Replacing again $l$ by $j$ we conclude that

$$
\sup \left\{d_{\widetilde{Y}}\left(\tilde{\varphi}_{i}^{j}(\tilde{x}), \tilde{\varphi}_{i}(\tilde{x})\right): \tilde{x} \in \widetilde{Z}^{j}, j \in\{1, \ldots, k\}\right\} \leqslant d_{i} .
$$

Because $d_{i} \rightarrow 0$ as $i \rightarrow \infty$ we thus recover [25, (2.6xiv)] as well, in our setting.

Returning to the sequence of maps $\tilde{\varphi}_{i}$ defined in (3.9) we apply the inequality in [25, Proposition 2.5.2] to the measures $\nu=\nu_{\tilde{x}}$ and $\nu^{\prime}=\nu_{\tilde{y}}$ on $\{1, \ldots, k\}$ (cf. (3.8)) and the maps $f, h$ given by $f(j)=\tilde{\varphi}_{i}^{j}(\tilde{x}), h(j)=\tilde{\varphi}_{i}^{j}(\tilde{y})$, whereby $j \in\{1, \ldots, k\}$ and $\tilde{x}, \tilde{y} \in \widetilde{X}$. Neglecting the negative terms on the right of the quoted inequality we obtain (by a slight deviation from the argument in [25, pp. 650-652])

$$
P^{2} \leqslant Q^{2}+2 P R \leqslant Q^{2}+\tau P^{2}+\tau^{-1} R^{2} \quad \text { for } 0<\tau \leqslant 1,
$$


where $P, Q, R$ are defined as follows, as functions of $\tilde{x}, \tilde{y} \in \tilde{X}$ with $d_{\widetilde{X}}(\tilde{x}, \tilde{y}) \leqslant \varrho:$

$$
\begin{aligned}
P & =d_{\tilde{Y}}\left(\tilde{\varphi}_{i}(\tilde{x}), \tilde{\varphi}_{i}(\tilde{y})\right) \quad(\text { to be estimated }), \\
Q & =\left(\sum_{j=1}^{k} \tilde{\eta}^{j}(\tilde{x}) d_{\tilde{Y}}^{2}\left(\tilde{\varphi}_{i}^{j}(\tilde{x}), \tilde{\varphi}_{i}^{j}(\tilde{y})\right)\right)^{1 / 2} \leqslant \sup _{j \in J(\tilde{x})} d_{\widetilde{Y}}\left(\tilde{\varphi}_{i}^{j}(\tilde{x}), \tilde{\varphi}_{i}^{j}(\tilde{y})\right) \\
& \leqslant C \sqrt{E\left(\psi_{i}\right)} d_{\tilde{X}}^{\alpha}(\tilde{x}, \tilde{y}), \\
R & =\sum_{j=1}^{k} d_{\widetilde{Y}}\left(\tilde{\varphi}_{i}^{j}(\tilde{y}), \tilde{\varphi}_{i}(\tilde{y})\right)\left|\tilde{\eta}^{j}(\tilde{y})-\tilde{\eta}^{j}(\tilde{x})\right| \leqslant C_{6} d_{i} d_{\widetilde{X}}(\tilde{x}, \tilde{y}) .
\end{aligned}
$$

The estimates of $Q$ and $R$ are uniform in $i, j$ and in $\tilde{x}, \tilde{y} \in \tilde{X}$ with $d_{\widetilde{X}}(\tilde{x}, \tilde{y}) \leqslant$ $\varrho$. The former estimate of $Q$ follows from $\sum_{j} \tilde{\eta}^{j}=1$. For the latter estimate of $Q$ note that, for any $\tilde{x} \in \tilde{X}$ and any $j \in J(\tilde{x})$, we have $x \in Z^{j}$ for $x:=p_{X}(\tilde{x})$. It follows by (3.10) that $y \in W^{j}$ for $y:=p_{X}(\tilde{y})$ because $d_{X}(x, y) \leqslant d_{\widetilde{X}}(\tilde{x}, \tilde{y}) \leqslant \varrho \leqslant \delta_{0}$. Consequently, $\tilde{x}, \tilde{y} \in \widetilde{W}^{j}$, and (3.11) applies as stated. Finally, the estimate of $R$ holds because $\tilde{\eta}^{j}=\eta^{j} \circ p_{X} \in \operatorname{Lip}(\widetilde{X})$ and $d_{\widetilde{Y}}\left(\tilde{\varphi}_{i}^{j}(\tilde{y}), \tilde{\varphi}_{i}(\tilde{y})\right) \leqslant d_{i}$ for $\tilde{y} \in \widetilde{Z}^{j}$, by $(3.21)$; and since $\left|\tilde{\eta}^{j}(\tilde{y})-\tilde{\eta}^{j}(\tilde{x})\right|=$ $\left|\eta^{j}(y)-\eta^{j}(x)\right|$ and $d_{\tilde{X}}(\tilde{x}, \tilde{y})=d_{X}(x, y)$, we may take $C_{6}=\sum_{1}^{k}\left\|\eta^{j}\right\|_{\text {Lip }}$.

Because $\alpha, \tau \leqslant 1$ and $d_{\tilde{X}}(\tilde{x}, \tilde{y}) \leqslant \varrho$ it follows from (3.19), (3.22), and the above estimates of $Q$ and $R$ that

$$
\begin{aligned}
(1-\tau) d_{\widetilde{Y}}^{2}\left(\tilde{\varphi}_{i}(\tilde{x}), \tilde{\varphi}_{i}(\tilde{y})\right) & \leqslant Q^{2}+\tau^{-1} R^{2} \\
& \leqslant\left(C^{2} E\left(\psi_{i}\right)+\tau^{-1} C_{6}^{2} d_{i}^{2} \delta_{0}^{2-2 \alpha}\right) d_{\tilde{X}}^{2 \alpha}(\tilde{x}, \tilde{y}) \\
& \leqslant C_{7} \tau^{-1} E\left(\psi_{i}\right) d_{\tilde{X}}^{2 \alpha}(\tilde{x}, \tilde{y})
\end{aligned}
$$

for $\tilde{x}, \tilde{y} \in \tilde{X}$ with $d_{\widetilde{X}}(\tilde{x}, \tilde{y}) \leqslant \varrho \leqslant \delta_{0}$ because $1 \leqslant \tau^{-1}$ and $d_{\widetilde{X}}(\tilde{x}, \tilde{y}) \leqslant \delta_{0}^{1-\alpha} d_{\widetilde{X}}^{\alpha}$ $(\tilde{x}, \tilde{y})$. (By the latter estimate (3.19) we may take $C_{7}=C^{2}+C_{5}^{2} C_{6}^{2} \delta_{0}^{2-2 \alpha}$ ). Taking now $\tau=\frac{1}{2}$ in (3.23) and recalling that the convergent sequences $\left(E\left(\psi_{i}\right)\right)$ and $\left(d_{i}^{2}\right)$ are bounded, we conclude that the $\tilde{\varphi}_{i}$ indeed are uniformly Hölder equicontinuous. It follows that the $\tilde{\varphi}_{i}$ are $\chi_{*^{-}}$equivariant (see the end of Step 1), and their projections $\varphi_{i} \in \mathcal{H}$ are likewise (uniformly) Hölder equicontinuous.

Step 3. The $\chi_{*}$-equivariant sequence $\left(\tilde{\varphi}_{i}\right)$ is minimizing. For $x, y \in X$ with $d_{X}(x, y) \leqslant \varrho$ choose $\tilde{x} \in p_{X}^{-1}(x), \tilde{y} \in p_{X}^{-1}(y)$ so that $d_{\tilde{X}}(\tilde{x}, \tilde{y})=d_{X}(x, y)$ $\leqslant \varrho$ (noting that $\varrho \leqslant \delta_{0} \leqslant r_{X}$, cf. the paragraph containing (2.2)). Inserting the definitions of $Q$ and $R$ in the first inequality (3.23) we obtain for $0<$ 
$\tau<1$

$$
\begin{aligned}
& (1-\tau) d_{\tilde{Y}}^{2}\left(\tilde{\varphi}_{i}(\tilde{x}), \tilde{\varphi}_{i}(\tilde{y})\right) \leqslant \sum_{j=1}^{k} \tilde{\eta}^{j}(\tilde{x}) d_{\tilde{Y}}^{2}\left(\tilde{\varphi}_{i}^{j}(\tilde{x}), \tilde{\varphi}_{i}^{j}(\tilde{y})\right) \\
& \quad+\tau^{-1}\left(\sum_{j \in J(\tilde{y})} d_{\tilde{Y}}^{2}\left(\tilde{\varphi}_{i}^{j}(\tilde{y}), \tilde{\varphi}_{i}(\tilde{y})\right)\right)\left(\sum_{j \in J(\tilde{y})}\left|\tilde{\eta}^{j}(\tilde{y})-\tilde{\eta}^{j}(\tilde{x})\right|^{2}\right)
\end{aligned}
$$

by Cauchy's inequality. Note at this point that, in the definition of $R$, it suffices to add over $j \in J(\tilde{y})$ (see text following $(3.20)$ ); for if $j \neq J(\tilde{y}$ ) then $\tilde{y} \notin \widetilde{Z}^{j}$, hence $\tilde{y}, \tilde{x} \notin \operatorname{supp} \tilde{\eta}^{j}$, and so $\tilde{\eta}^{j}(\tilde{y})=\tilde{\eta}^{j}(\tilde{x})=0$. Consider an $m$-simplex $\tilde{s}\left(=\tilde{s}_{\nu}\right)$ of the "fundamental domain" $\widetilde{F} \subset \widetilde{X}$. After integrating (3.24) over $\tilde{y} \in B_{\tilde{s}}(\tilde{x}, \varepsilon)$ for $\varepsilon \leqslant \varrho$, and next over $\tilde{x} \in \tilde{s}$, dividing by $c_{m} \varepsilon^{m+2}$, and letting $\varepsilon \rightarrow 0$ whilst invoking (2.18), (2.21), and (2.23) (applied with $\tilde{X}$ replaced by $\tilde{s})$ we obtain from $(3.24)$

$$
(1-\tau) E\left(\tilde{\varphi}_{i} \mid \tilde{s}\right) \leqslant \sum_{j=1}^{k} \int_{\tilde{s}} e\left(\tilde{\varphi}_{i}^{j}\right) \tilde{\eta}^{j} d \tilde{\mu}+C_{8} \tau^{-1} d_{i}^{2}
$$

with $C_{8}=k \sum_{1}^{k} \int_{s}\left|\nabla \eta^{j}\right|^{2} d \mu$, noting moreover that $d_{\widetilde{Y}}\left(\tilde{\varphi}_{i}^{j}, \tilde{\varphi}_{i}\right) \leqslant d_{i}$ on $\widetilde{Z}^{j}$ according to $(3.21)$.

Because the $\tilde{\eta}^{j}$ form a partition of unity on $\widetilde{X},(3.25)$ may be rewritten as follows after summation over the $m$-simplexes $\tilde{s}$ of $\widetilde{F}$ followed by projection, invoking (3.3):

$$
(1-\tau) E\left(\varphi_{i}\right) \leqslant E\left(\psi_{i}\right)+\sum_{j=1}^{k} \int_{X}\left(e\left(\varphi_{i}^{j}\right)-e\left(\psi_{i}\right)\right) \eta^{j} d \mu+C_{9} \tau^{-1} d_{i}^{2},
$$

where we may take $C_{9}$ equal to $C_{8}$ times the number of $m$-simplexes of $X$.

Still following [25] we proceed to show that the term in (3.26) with summation over $j$ approaches 0 as $i \rightarrow \infty$ because $\left(\varphi_{i}^{j}\right)_{i}$ and $\left(\psi_{i}\right)$ are $E$-minimizing sequences. Applying quadrilateral comparison [25, (2.1iv)] with $t=\frac{1}{2}$ and $\alpha=0$ to the $\chi_{*}$-equivariant midpoint map $\tilde{w}_{i}^{j}=\frac{1}{2} \tilde{\varphi}_{i}^{j}+\frac{1}{2} \tilde{\psi}_{i}$ : $s^{\circ} \rightarrow \widetilde{Y}$ we obtain after projection in view of the uniform equicontinuity of the $\chi_{*}$-equivariant maps $\tilde{\varphi}_{i}^{j}, \tilde{\psi}_{i}$, and $\widetilde{w}_{i}^{j}$ :

$$
\begin{aligned}
d_{Y}^{2}\left(w_{i}^{j}(x), w_{i}^{j}(y)\right) & \leqslant \frac{1}{2} d_{Y}^{2}\left(\varphi_{i}^{j}(x), \varphi_{i}^{j}(y)\right)+\frac{1}{2} d_{Y}^{2}\left(\psi_{i}(x), \psi_{i}(y)\right) \\
& -\frac{1}{4}\left(d_{Y}\left(\varphi_{i}^{j}(x), \varphi_{i}^{j}(y)\right)-d_{Y}\left(\psi_{i}(x), \psi_{i}(y)\right)\right)^{2}
\end{aligned}
$$


for $d_{\widetilde{X}}(\tilde{x}, \tilde{y})$ sufficiently small that $d_{\widetilde{Y}}\left(\tilde{w}_{i}^{j}(\tilde{x}), \tilde{w}_{i}^{j}(\tilde{y})\right) \leqslant r_{Y}$, etc.; cf. $(2.2)$ and subsequent text. It follows by $(2.18),(2.19)$, for any open $m$-simplex $s^{\circ}$ of $X$ and any $f \in C_{c}\left(s^{\circ},[0,1]\right)$ that (up to a dimensional constant factor)

$$
\begin{aligned}
& \limsup _{\varepsilon \rightarrow 0} \frac{m+2}{4} \iint_{\substack{x, y \in s^{\circ} \\
d_{X}(x, y) \leqslant \varepsilon}}\left(\frac{d_{Y}\left(\varphi_{i}^{j}(x), \varphi_{i}^{j}(y)\right)}{\varepsilon}-\frac{d_{Y}\left(\psi_{i}(x), \psi_{i}(y)\right)}{\varepsilon}\right)^{2} f(x) \frac{d \mu(x) d \mu(y)}{\varepsilon^{m}} \\
& \quad \leqslant \int_{s^{\circ}}\left(\frac{1}{2} e\left(\varphi_{i}^{j}\right)+\frac{1}{2} e\left(\psi_{i}\right)-e\left(w_{i}^{j}\right)\right) f d \mu .
\end{aligned}
$$

According to $[25$, p. 648 (top)] the left-hand member of (3.27) equals

$$
\int_{S^{m-1}} \int_{s^{\circ}}\left(\left|\left(\varphi_{i}^{j}\right)_{*}(\omega)\right|-\left|\left(\psi_{i}\right)_{*}(\omega)\right|\right)^{2} f d \mu d \sigma(\omega),
$$

where for example $\left|\left(\psi_{i}\right)_{*}(\omega)\right|^{2}$ denotes the directional energy density of $\psi_{i} \in$ $\mathcal{E}\left(s^{\circ}, Y\right)$ (cf. [25, Theorem 1.9 .6 and eq. (1.10v)]) in the direction corresponding to $\omega \in S^{m-1}$ (the unit sphere in $\mathbb{R}^{m}$ with surface measure $\sigma$ ). On the other hand, by $[25,(1.10 \mathrm{ii})]$ applied to the maps $\varphi_{i}^{j}$ and $\psi_{i}$, and the function $\eta^{j} f$ (in place of $f$ ):

$$
\begin{aligned}
\int_{s^{\circ}} & \left(e\left(\varphi_{i}^{j}\right)-e\left(\psi_{i}\right)\right) \eta^{j} f d \mu \\
\quad= & \int_{s^{\circ}} \int_{S^{m-1}}\left(\left|\left(\varphi_{i}^{j}\right)_{*}(\omega)\right|^{2}-\left|\left(\psi_{i}\right)_{*}(\omega)\right|^{2}\right) d \sigma(\omega) \eta^{j} f d \mu .
\end{aligned}
$$

By the Cauchy-Schwarz inequality applied to the product

$$
\left(\left|\left(\varphi_{i}^{j}\right)_{*}(\omega)\right|+\left|\left(\psi_{i}\right)_{*}(\omega)\right|\right) \eta^{j} \sqrt{f} \times\left(\left|\left(\varphi_{i}^{j}\right)_{*}(\omega)\right|-\left|\left(\psi_{i}\right)_{*}(\omega)\right|\right) \sqrt{f}
$$

the square of the right-hand member of (3.29) is no bigger than

$$
\begin{gathered}
\int_{s^{\circ}} \int_{S^{m-1}}\left(\left|\left(\varphi_{i}^{j}\right)_{*}(\omega)\right|+\left|\left(\psi_{i}\right)_{*}(\omega)\right|\right)^{2} d \sigma(\omega)\left(\eta^{j}\right)^{2} f d \mu \times \\
\int_{s^{\circ}} \int_{S^{m-1}}\left(\left|\left(\varphi_{i}^{j}\right)_{*}(\omega)\right|-\left|\left(\psi_{i}\right)_{*}(\omega)\right|\right)^{2} d \sigma(\omega) f d \mu \\
\leqslant 2\left(E\left(\varphi_{i}^{j}\right)+E\left(\psi_{i}\right)\right) \int_{s^{\circ}}\left(\frac{1}{2} e\left(\varphi_{i}^{j}\right)+\frac{1}{2} e\left(\psi_{i}\right)-e\left(w_{i}^{j}\right)\right) f d \mu
\end{gathered}
$$

in view of (3.27) and (3.28), inserting $\left(\eta^{j}\right)^{2} f \leqslant 1$, using $(a+b)^{2} \leqslant 2 a^{2}+2 b^{2}$, and again invoking $[25,1.10 \mathrm{ii}]$. In the limit as $f \rightarrow 1$ pointwise through 
an increasing sequence we altogether obtain (up to a dimensional constant factor) by dominated convergence and by (2.22), (3.7), (3.29), and (3.30),

$$
\int_{s^{\circ}}\left(e\left(\varphi_{i}^{j}\right)-e\left(\psi_{i}\right)\right) \eta^{j} d \mu \leqslant 2 \sqrt{E\left(\psi_{i}\right)} \sqrt{E\left(\psi_{i}\right)-E\left(w_{i}^{j}\right)} .
$$

Because the midpoint map $\tilde{w}_{i}^{j}=\frac{1}{2} \tilde{\varphi}_{i}^{j}+\frac{1}{2} \tilde{\psi}_{i}$ is $\chi_{*}$-equivariant along with $\tilde{\varphi}_{i}^{j}$ and $\tilde{\psi}_{i}$, its projection $w_{i}^{j}$ belongs to $\mathcal{H}$ and so has energy $\geqslant E_{0}$. Adding over all $m$-simplexes $s$ of $X$ we conclude that the sum term in (3.26) indeed has the limit 0 as $i \rightarrow \infty$. By (3.26) we therefore obtain

$$
\limsup _{i \rightarrow \infty}\left[(1-\tau) E\left(\varphi_{i}\right)\right] \leqslant E_{0}
$$

and hence $\lim \sup _{i} E\left(\varphi_{i}\right) \leqslant E_{0}$, by letting $\tau \rightarrow 0$. In the opposite direction, $E_{0} \leqslant E\left(\varphi_{i}\right)$ by definition of $E_{0}$. Consequently, $\lim _{i} E\left(\varphi_{i}\right)=E_{0}$, and the sequence $\left(\tilde{\varphi}_{i}\right)$ is indeed minimizing; cf. (3.4) and (3.5).

Step 4. Existence and harmonicity of E-minimizers. Using the above minimizing sequence of $\chi_{*}$-equivariant maps $\tilde{\varphi}_{i}: \widetilde{X} \rightarrow \widetilde{Y}$ we commence as indicated in the proof of $\left[25\right.$, Theorem 2.7.1]. Since $\left(\tilde{\varphi}_{i}\right)$ is uniformly Hölder equicontinuous, so is the sequence of projected maps $\varphi_{i}: X \rightarrow Y$. By Ascoli's theorem, a subsequence, again denoted $\left(\varphi_{i}\right)$, converges uniformly to a continuous map $\varphi: X \rightarrow Y$. Fix $i \in\{1,2, \ldots\}$ so that $d_{Y}\left(\varphi(x), \varphi_{i}(x)\right) \leqslant r_{Y}$ for all $x \in X$, with $r_{Y}$ from the paragraph containing (2.2). Also fix $x_{0} \in X$ and $\tilde{x}_{0} \in p_{X}^{-1}\left(x_{0}\right)$. Let $\tilde{\varphi}: \widetilde{X} \rightarrow \widetilde{Y}$ denote the unique lift of $\varphi: X \rightarrow Y$ such that $d_{\widetilde{Y}}\left(\tilde{\varphi}\left(\tilde{x}_{0}\right), \tilde{\varphi}_{i}\left(\tilde{x}_{0}\right)\right)=d_{Y}\left(\varphi\left(x_{0}\right), \varphi_{i}\left(x_{0}\right)\right)\left(\leqslant r_{Y}\right)$. Because $\tilde{\varphi}$ and $\tilde{\varphi}_{i}$ are continuous and $\Gamma_{Y}$ is discrete, it follows that $d_{\tilde{Y}}\left(\tilde{\varphi}(\tilde{x}), \tilde{\varphi}_{i}(\tilde{x})\right) \leqslant r_{Y}$ for every $\tilde{x} \in \tilde{X}$. We show that $\tilde{\varphi}$ is $\chi_{*}$-equivariant. For any $\gamma \in \Gamma_{X}$ the continuous map $\chi_{*}(\gamma)^{-1} \circ \tilde{\varphi} \circ \gamma$ from $\widetilde{X}$ to $\widetilde{Y}$ is likewise a lift of $\varphi$ because

$$
p_{Y} \circ\left(\chi_{*}(\gamma)^{-1} \circ \tilde{\varphi} \circ \gamma\right)=p_{Y} \circ \tilde{\varphi} \circ \gamma=\varphi \circ p_{X} \circ \gamma=\varphi \circ p_{X}
$$

cf. (2.3). Because $\tilde{\varphi}_{i}$ is $\chi_{*}$-equivariant (see end of Step 2), we have

$$
d_{\widetilde{Y}}\left(\left(\chi_{*}(\gamma)^{-1} \circ \tilde{\varphi} \circ \gamma\right)(\tilde{x}), \tilde{\varphi}_{i}(\tilde{x})\right)=d_{\tilde{Y}}\left(\tilde{\varphi}(\gamma(\tilde{x})), \tilde{\varphi}_{i}(\gamma(\tilde{x}))\right) \leqslant r_{Y}
$$

for any $\tilde{x} \in \tilde{X}$, noting that $\chi_{*}(\gamma)$ and $\gamma$ are isometries of $\tilde{Y}$ and $\tilde{X}$, respectively. By uniqueness of $\tilde{\varphi}$ we have $\chi_{*}(\gamma)^{-1} \circ \tilde{\varphi} \circ \gamma=\tilde{\varphi}$, and so indeed $\tilde{\varphi}$ is $\chi_{*}$-equivariant. Its projection $\varphi$ therefore belongs to $\mathcal{H}$. From the uniform convergence $\varphi_{i} \rightarrow \varphi$ we infer that $\left.D^{2}\left(\varphi_{i}, \varphi\right)=\int_{X} d_{Y}^{2}\left(\varphi_{i}(x)\right), \varphi(x)\right) d \mu(x) \rightarrow 0$ because $\mu(X)<\infty$. This means that $\varphi_{i} \rightarrow \varphi$ in $L^{2}(X, Y)$ (cf. the paragraph 
following the one containing (2.17)), and consequently

$$
E(\varphi) \leqslant \liminf _{i \rightarrow \infty} E\left(\varphi_{i}\right)=E_{0}
$$

by [9, Lemma 9.1]. Thus $\varphi$ is an E-minimizer relative to $\mathcal{H}$.

For the proof that every $E$-minimizer $\varphi$ relative to $\mathcal{H}$ is harmonic in $X$ in the sense of (c), and hence of (a) and (b) in Remark 2.6, cf. Lemma 2.7, fix a point $p \in X$. Since $Y$ is complete and has nonpositive curvature, $\varphi(p)$ has a convex open neighbourhood $V$ in $Y$ such that $V$ is a $\operatorname{CAT}(0)$ space, [2, p. $159 \mathrm{f}]$. Thus any two points $y, z$ of $V$ can be joined by a unique constant-speed geodesic $[0,1] \ni t \mapsto \zeta(y, z ; t) \in V,[2$, pp. 4, 160]; and $\zeta$ depends continuously on $(y, z, t) \in V \times V \times[0,1],[2$, p. $158 \mathrm{ff}]$. For a given neighbourhood $N$ of $p$ in $X$ choose a connected open set $U \Subset X$ with $p \in U \subset N$ so that $\varphi(U) \Subset V$. Consider any continuous map $\psi \in \mathcal{E}(X, Y)$ such that $\psi=\varphi$ in $X \backslash U$ and $\psi(U) \subset V$. We prove that $\psi \in \mathcal{H}$, hence $E(\psi) \geqslant E(\varphi)$, and so, by varying $p$ and $N$, that $\varphi$ satisfies (c) in Remark 2.6. Define $\Psi: X \times[0,1] \rightarrow Y$ by

$$
\Psi(x, t)= \begin{cases}\zeta(\varphi(x), \psi(x) ; t) & \text { for } x \in U, t \in[0,1] \\ \varphi(x)=\psi(x) & \text { for } x \in X \backslash U, t \in[0,1]\end{cases}
$$

For $U \ni x \rightarrow x_{0} \in \partial U$ and $[0,1] \ni t \rightarrow t_{0} \in[0,1]$ we have $\varphi(x) \rightarrow \varphi\left(x_{0}\right) \in V$ and $\psi(x) \rightarrow \psi\left(x_{0}\right)=\varphi\left(x_{0}\right)$, and hence $\zeta(\varphi(x), \psi(x) ; t) \rightarrow \zeta\left(\varphi\left(x_{0}\right), \varphi\left(x_{0}\right) ; t_{0}\right)$ $=\varphi\left(x_{0}\right)$, that is, $\Psi(x, t) \rightarrow \Psi\left(x_{0}, t_{0}\right)$. Thus $\Psi$ is continuous both relative to $\bar{U}$ and to $X \backslash U$, and hence on all of $X$. Clearly $\Psi(x, 0)=\varphi(x), \Psi(x, 1)=$ $\psi(x)$; so indeed $\psi \in \mathcal{H}$, and $\varphi$ is harmonic according to Remark 2.6. It may be added that every minimizing $\chi_{*}$-equivariant map $\tilde{\varphi}: \widetilde{X} \rightarrow \widetilde{Y}$ likewise is harmonic, harmonicity being a local property by Lemma 2.7 .

Step 5. The uniqueness assertion. We begin by proving that any two $\chi_{*}$-equivariant energy minimizers $\tilde{\varphi}_{0}, \tilde{\varphi}_{1}$ have constant distance $d_{\widetilde{Y}}\left(\tilde{\varphi}_{0}(\cdot)\right.$, $\left.\tilde{\varphi}_{1}(\cdot)\right)$. First note that the restriction of the distance function $d_{\widetilde{Y}}\left(\tilde{\varphi}_{0}, \tilde{\varphi}_{1}\right)$ to each $m$-simplex $\tilde{s}\left(=\tilde{s}_{\nu}\right)$ of the "fundamental domain" $\widetilde{F}$ is of class $\mathcal{E}(\tilde{s}, \mathbb{R})=W^{1,2}(\tilde{s})$; see for example $[14$, p. 252 (bottom)]. For any $t \in[0,1]$ the restriction of the map $\tilde{\varphi}_{t}:=(1-t) \tilde{\varphi}_{0}+t \tilde{\varphi}_{1}$ (with the usual abuse of notation) to each $\tilde{s}$ is of class $\mathcal{E}(\tilde{s}, \widetilde{Y})$ by the energy convexity property

$$
E\left(\tilde{\varphi}_{t} \mid \tilde{s}\right) \leqslant(1-t) E\left(\tilde{\varphi}_{0} \mid \tilde{s}\right)+t E\left(\tilde{\varphi}_{1} \mid \tilde{s}\right)-t(1-t) \int_{\tilde{s}}\left|\nabla d_{\widetilde{Y}}\left(\tilde{\varphi}_{0}, \tilde{\varphi}_{1}\right)\right|^{2} d \tilde{\mu}
$$

(valid for any two maps $\tilde{\varphi}_{0}, \tilde{\varphi}_{1} \in \mathcal{E}(\tilde{s}, \tilde{Y})$ ), cf. [9, eq. (11.2); 25, 2.2iv]. Also, each $\tilde{\varphi}_{t}$ is $\chi_{*}$-equivariant, $\gamma \in \Gamma_{X}$ and $\chi_{*}(\gamma) \in \Gamma_{Y}$ being isometries. For the 
projection $\varphi_{t} \in \mathcal{H}$ of $\tilde{\varphi}_{t}$ we therefore have from (3.4) after summation over all $m$-simplexes $\tilde{s}$ of $\tilde{F}$

$$
E_{0} \leqslant E\left(\varphi_{t}\right) \leqslant E_{0}-t(1-t) \int_{\widetilde{F}}\left|\nabla d_{\widetilde{Y}}\left(\tilde{\varphi}_{0}, \tilde{\varphi}_{1}\right)\right|^{2} d \tilde{\mu} \leqslant E_{0},
$$

and hence $\int_{\widetilde{F}}\left|\nabla d_{\widetilde{Y}}\left(\tilde{\varphi}_{0}, \tilde{\varphi}_{1}\right)\right|^{2} d \tilde{\mu}=0$, by taking $0<t<1$. It follows that $d_{\widetilde{Y}}\left(\tilde{\varphi}_{0}, \tilde{\varphi}_{1}\right)$ is constant in any $\tilde{s}$. Because every open star $\widetilde{S}$ of $\widetilde{X}$ is contained in some open ball of radius $r_{X}$ (cf. the beginning of the proof of the present Theorem 3.2), $\widetilde{F}$ can be chosen so as to contain $\widetilde{S}$, cf. text between (3.12) and $(3.13))$; hence $d_{\widetilde{Y}}\left(\tilde{\varphi}_{0}, \tilde{\varphi}_{1}\right)$ equals a constant $\tilde{d}$ in $\widetilde{S}$. Thus $d_{\widetilde{Y}}\left(\tilde{\varphi}_{0}, \tilde{\varphi}_{1}\right)$ is locally constant in $\widetilde{X}$, and therefore constant in all of $\widetilde{X}$ (connected). By projection, this leads to Hartman's uniqueness result [21] in the present setting, as stated after the formulation of Theorem 3.2: If $\tilde{d}<r_{Y}$ then $\tilde{d}=d_{\widetilde{Y}}\left(\tilde{\varphi}_{0}(\tilde{x}), \tilde{\varphi}_{1}(\tilde{x})\right)=d_{Y}\left(\varphi_{0}(x), \varphi_{1}(x)\right)$ by $(2.2)$ and subsequent text, and the geodesic segment $t \mapsto \varphi_{t}(x)$ therefore becomes minimizing. Finally, if $\varphi_{0}(x)=\varphi_{1}(x)$ at some point $x$ then $\tilde{d}=0$ and consequently $\varphi_{0}=\varphi_{1}$.

Remark 3.3. Under the hypotheses on $X$ and $Y$ in Theorem 3.2, Hölder continuity of a harmonic map $\varphi: X \rightarrow Y$ (cf. Proposition 3.1, now with $X$ compact) holds in the global uniform sense that there are constants $C, \alpha>0$ depending only on $\left(X, \Lambda_{X}\right)$ such that

$$
d_{Y}(\varphi(x), \varphi(y)) \leqslant C \sqrt{E(\varphi)} d_{X}^{\alpha}(x, y), \quad x, y \in X .
$$

With the extra requirement that $d_{X}(x, y) \leqslant \varrho$ for a suitable constant $\varrho>0$, this holds according to Proposition $3.1, X$ being compact. Since $\left(X, d_{X}\right)$ is a geodesic space [9, Proposition 4.1(b)], we may join any two points $x$ and $y$ of $X$ by a geodesic $\eta$ in $X$ of length $d_{X}(x, y) \leqslant$ the diameter of $\left(X, d_{X}\right)$, and it suffices to apply the version of (3.34) for $d_{X}(x, y) \leqslant \varrho$ after first subdividing $\eta$ into a fixed number of arcs of length not exceeding $\varrho$ (cf. the analogous paragraph following the one containing (2.12)).

Remark 3.4. We shall need in Section 5 that, for variable Riemannian metric $g$ on $X$, the constants $r_{X}, C, \alpha, \varrho, \delta_{0}$, and $C_{1}$ through $C_{9}$ in the proof of Theorem 3.2 can be chosen so as to depend only on $X, \Lambda_{X}$ (as already noted concerning $C, \alpha, \varrho$ in Proposition 3.1). If $r_{X}^{e}$ denotes a value of $r_{X}$ relative to the Euclidean Riemannian metric $g^{e}$ on $X$ (see [9, p. 49]), then we may take $r_{X}=\Lambda_{X}^{-1} r_{X}^{e}$ for the constant $r_{X}$ relative to a given Riemannian metric $g$ on $X$ with ellipticity constant $\Lambda_{X}$; cf. [9, (4.1)]. This is because 
the intrinsic Riemannian distances $d_{X}$ and $d_{X}^{e}$ satisfy

$$
\Lambda_{X}^{-1} d_{X}^{e} \leqslant d_{X} \leqslant \Lambda_{X} d_{X}^{e}
$$

cf. $\left[9\right.$, p. 52 (bottom)]. It follows that $B_{X}\left(x, \Lambda_{X}^{-1} r_{X}^{e}\right) \subset B_{X}^{e}\left(x, r_{X}^{e}\right)$ for $x \in X$; and since $p_{X} \mid B_{X}^{e}\left(x, r_{X}^{e}\right)$ is an isometry, by (2.2), we infer that $p_{X} \mid B_{X}(x$, $\left.\Lambda_{X}^{-1} r_{X}^{e}\right)$ is injective and hence likewise an isometry; cf. [31, Proposition 3.5.7]. In this way, $r_{X}$ depends on $X, \Lambda_{X}$ only. In view of (3.35) the same holds for $\delta_{0}$, cf. (3.10). It is easily verified that $C_{1}$ through $C_{9}$ likewise depend on $\left(X, \Lambda_{X}\right)$ only.

\section{The Dirichlet homotopy problem}

We continue considering a compact admissible Riemannian polyhedron $(X, g)$, but now with $b X \neq \varnothing$; and a compact geodesic space $Y$ of nonpositive curvature. We therefore have the equivalent definitions (a), (b), and (c) of harmonicity of a continuous map $\varphi: X \rightarrow Y$, formulated in Remark 2.6 above, cf. Lemma 2.7.

Let $\Omega$ denote a nonvoid connected open subset of $X$, and denote by $\partial \Omega$ the topological boundary and by $\bar{\Omega}$ the closure of $\Omega$ in $X$. Note that $X$ is a harmonic space in the sense of Brelot [4, Part IV; $5, \S 3.1]$; see [9, Theorem 7.1]. We require throughout that $\Omega$ is regular in the sense of potential theory and that $\Omega \cap b X$ is not dense in $b X$. Recall that $\Omega$ is said to be regular if $\partial \Omega \neq \varnothing$ and every continuous function $\partial \Omega \rightarrow \mathbb{R}$ has a (necessarily unique) continuous extension $\bar{\Omega} \rightarrow \mathbb{R}$ which is harmonic in $\Omega$. Equivalently, every point $x \in \partial \Omega$ shall be regular, that is, $X \backslash \Omega$ shall be nonthin at $x$ in the sense of Brelot [4, p. 137], or see [1, Theorem 7.5.1; $5, \S 6.3]$. In particular, $X \backslash b X$ is always regular. The boundary of a regular domain can, however, be rather "wild"; cf. [14, Example 2].

We proceed to show that the Dirichlet homotopy problem for harmonic maps has a unique solution. For any map $\chi \in C(X, Y)$ (i.e., $\chi: X \rightarrow Y$ is continuous) write

$$
C_{\chi}(\Omega, Y)=\{\varphi \in C(X, Y): \varphi=\chi \text { on } X \backslash \Omega\}
$$

Denote by $\mathcal{H}_{\chi}(\Omega, Y)$ the class of all maps in $C_{\chi}(\Omega, Y)$ which are homotopic with $\chi$, the homotopy being through maps of class $C_{\chi}(\Omega, Y)$. Denote by $\mathcal{H}$ the homotopy class of all maps in $C(X, Y)$ which are freely homotopic to $\chi$; then $\mathcal{H}_{\chi}(\Omega, Y) \subset \mathcal{H}$. 
Theorem 4.1. Let $\Omega$ be a connected regular open subset of a compact admissible Riemannian polyhedron $(X, g)$ with $b X \neq \varnothing$, and suppose that $\Omega \cap b X$ is not dense in $b X$. Let $\left(Y, d_{Y}\right)$ be a compact geodesic space of nonpositive curvature; and let a continuous map $\chi: X \rightarrow Y$ of finite energy be given. Then there exists in $\mathcal{H}_{\chi}(\Omega, Y)$ a unique map $\varphi_{0}$ of least energy, and $\varphi_{0} \mid \Omega$ is harmonic and (locally uniformly) Hölder continuous.

In earlier work on the Dirichlet problem for harmonic maps (for example in $[13,25])$, the present regular domain $\Omega$ was replaced by the particular regular domain $\Omega=X \backslash b X$. For that case, Theorem 4.1 can be established alternatively by modifying the proof given below, notably by using [13, Theorem 1] (as in the proof of Theorem 3.2 above) in place of [14, Theorem 1], needed in Step 2 of the present proof.

Proof of Theorem 4.1. Again, we pass to the universal covers $\widetilde{X}$ and $\tilde{Y}$. In view of [42, Theorem 35, p. 317] we may assume that every simplex of $X$ has diameter $\leqslant r_{X}$ from (2.2), and from (2.8) in the proof of Proposition 2.2. According to [18, Theorem 1 and Corollary 3] the connected open set $\Omega \subset X$ is an admissible Riemannian polyhedron (along with $X$ ) with a triangulation $T_{\Omega}$ such that every simplex of $\left(\Omega, T_{\Omega}\right)$ is a subset of some simplex of $X$, and hence has diameter $\leqslant r_{X}$. Define $\widetilde{\Omega}=p_{X}^{-1}(\Omega)$.

Henceforth, until Step 4 below, we suppose that $\Omega$ is not dense in $X$.

Step 1. A quasi-equivariant minimizer $\tilde{\varphi}_{0}$. Fix a base point $x_{0} \in X \backslash \Omega$ and a point $\tilde{x}_{0} \in p_{X}^{-1}\left(x_{0}\right)$. For any $\varphi \in \mathcal{H}_{\chi}(\Omega, Y)$ there exists a continuous map $\Phi: X \times[0,1] \rightarrow Y$ such that $\Phi(x, 0)=\chi(x)$ and $\Phi(x, 1)=\varphi(x)$ for $x \in X$, and that $\Phi(x, t)=\chi(x)$ for $x \in X \backslash \Omega$ and $t \in[0,1]$. Because $X \times[0,1]$ has the same properties as listed in Section 2 for $X$ itself, allowing lifting of continuous maps into $Y$, there exists a lift $\widetilde{\Phi}: \widetilde{X} \times[0,1] \rightarrow \widetilde{Y}$. For each $t \in[0,1]$ the restriction $\widetilde{\Phi}(\cdot, t): \widetilde{X} \rightarrow \widetilde{Y}$ is a lift of $\Phi(\cdot, t): X \rightarrow Y$. In particular, $\tilde{\chi}:=\widetilde{\Phi}(\cdot, 0)$ is a lift of $\chi$. For $\tilde{x} \in \widetilde{X} \backslash \widetilde{\Omega}$ we have $x:=p_{X}(\tilde{x}) \in$ $X \backslash \Omega$, and hence $\widetilde{\Phi}(\tilde{x}, t) \in p_{Y}^{-1}(\Phi(x, t))=p_{Y}^{-1}(\chi(x))$ for $t \in[0,1]$. Because $\Gamma_{Y}$ is discrete and $\widetilde{\Phi}(\tilde{x}, \cdot)$ is continuous it follows that $\widetilde{\Phi}(\tilde{x}, t)$ is independent of $t \in[0,1]$ for each $\tilde{x} \in \widetilde{X} \backslash \widetilde{\Omega}$. Hence $\tilde{\varphi}(\tilde{x}):=\widetilde{\Phi}(\tilde{x}, 1)=\widetilde{\Phi}(\tilde{x}, 0)=\tilde{\chi}(\tilde{x})$ for $\tilde{x} \in \tilde{X} \backslash \widetilde{\Omega}$, in particular $\tilde{\varphi}\left(\tilde{x}_{0}\right)=\tilde{\chi}\left(\tilde{x}_{0}\right)$. Since $\varphi \in \mathcal{H}_{\chi}(\Omega, Y) \subset \mathcal{H}$ there is accordingly a unique homomorphism $\chi_{*}: \Gamma_{X} \rightarrow \Gamma_{Y}$ such that $\tilde{\varphi}$ is $\chi_{*^{-}}$ equivariant, as in (3.2). Denote by $\mathcal{A}$ the class of all (quasicontinuous) maps $\tilde{\varphi} \in \mathcal{E}_{\mathrm{loc}}(\widetilde{X}, \widetilde{Y})$ which are quasi-equivariant in the sense that (3.2) holds q.e.:

$$
\tilde{\varphi} \circ \gamma=\chi_{*}(\gamma) \circ \tilde{\varphi} \quad \text { q.e. in } \tilde{X} \text { for all } \gamma \in \Gamma_{X} .
$$


Denote

$$
\mathcal{A}_{\tilde{\chi}}=\{\tilde{\varphi} \in \mathcal{A}: \tilde{\varphi}=\tilde{\chi} \text { q.e. in } \tilde{X} \backslash \widetilde{\Omega}\}
$$

Then $\tilde{\chi} \in \mathcal{A}_{\tilde{\chi}}$; in particular $\mathcal{A}_{\tilde{\chi}} \neq \varnothing$.

Every map $\tilde{\varphi} \in \mathcal{A}$ has a quasicontinuous projection $\varphi \in \mathcal{E}(X, Y)$. Indeed, (3.2) holds in $\widetilde{X} \backslash \widetilde{P}$ for some polar set $\widetilde{P} \subset \widetilde{X}$. Replacing $\widetilde{P}$ by the countable union of polar sets $\gamma(\widetilde{P}), \gamma \in \Gamma_{X}$, we arrange that $\widetilde{P}$ becomes $\Gamma_{X}$-invariant. For any $x \in X$ off the polar set $p_{X}(\tilde{P})$ define $\varphi(x)=\left(p_{Y} \circ \tilde{\varphi}\right)(\tilde{x})$ for $\tilde{x} \in$ $p_{X}^{-1}(x) \subset \widetilde{X} \backslash \widetilde{P}$; this does not depend on the choice of $\tilde{x}$ on account of (3.2). Finally, $\varphi \in \mathcal{E}_{\text {loc }}(X, Y)=\mathcal{E}(X, Y)$, and $\varphi$ is quasicontinuous along with $\tilde{\varphi}$, both because $p_{X}$ and $p_{Y}$ are local isometries.

Every continuous map $\tilde{\varphi} \in \mathcal{A}$ is $\chi_{*}$-equivariant in the sense of (3.2), that is, (4.1) holds everywhere in $\tilde{X}$. If even $\tilde{\varphi} \in \mathcal{A}_{\tilde{\chi}}$ then the continuous projection $\varphi: X \rightarrow Y$ is of class $C_{\chi}(\Omega, Y)$, and even of class $\mathcal{H}_{\chi}(\Omega, Y)$, as shown by geodesic homotopy; see the text following (3.2), and note that we now have $\Phi(x, t)=\chi(x)$ for $x \in X \backslash \Omega$.

For a given triangulation of $X$, and hence of $\tilde{X}$, consider a compact "fundamental domain" $\widetilde{F} \subset \widetilde{X}$ of $\Gamma_{X}$ as in the paragraph containing (3.3) and its predecessor. Thus $\widetilde{F}$ is a union of $m$-simplexes $\tilde{s}_{\nu}$ projected by $p_{X}$ isometrically on the $m$-simplexes $s_{\nu}$ of $X, \nu \in\{1, \ldots, n\}$. For $\varepsilon \leqslant r_{X}$ we have $B_{X}(x, \varepsilon)=p_{X}\left(B_{\widetilde{X}}(\tilde{x}, \varepsilon)\right)$ for all $\tilde{x} \in p_{X}^{-1}(x)$ according to $(2.2)$. Since $p_{Y}$ is nonexpanding, the projection $\varphi$ of a map $\tilde{\varphi} \in \mathcal{A}$ therefore satisfies $\varphi \mid s_{\nu} \in$ $L^{2}\left(s_{\nu}, Y\right)$ and hence $\varphi \in L^{2}(X, Y)$. Furthermore, $e_{\varepsilon}(\varphi) \circ p_{X} \leqslant e_{\varepsilon}(\tilde{\varphi})$, and hence $e(\varphi) \circ p_{X} \leqslant e(\tilde{\varphi})$ and $E(\varphi) \leqslant E(\tilde{\varphi} \mid \widetilde{F})=\sum_{\nu=1}^{n} E\left(\tilde{\varphi} \mid \tilde{s}_{\nu}\right)=\int_{\widetilde{F}} e(\tilde{\varphi}) d \tilde{\mu}$ $(<\infty)$; cf. Lemma $2.3(\mathrm{~b})$. If $\tilde{\varphi}$ is continuous then so is $\varphi$, and the sign $\leqslant$ can here be replaced in the last two cases by equality (as in (3.3)), $p_{Y}$ being locally isometric.

There exists a constant $c_{1}$ such that the following Poincaré-style inequality holds (cf. [6, Proposition 2.2]):

$$
\int_{\widetilde{F}}(u-\bar{u})^{2} d \tilde{\mu} \leqslant c_{1} \int_{\widetilde{F}}|\nabla u|^{2} d \tilde{\mu}
$$

for any function $u \in W_{\text {loc }}^{1,2}(\widetilde{X})$ which is essentially $\Gamma_{X}$-invariant: $u \circ \gamma=u$ $\mu$-a.e. in $\widetilde{X}$ for every $\gamma \in \Gamma_{X}$. Here $\bar{u}=\tilde{\mu}(\widetilde{F})^{-1} \int_{\widetilde{F}} u d \tilde{\mu}$ denotes the average of $u$ over $\widetilde{F}$. We prove (4.2) in the equivalent projected form where $\widetilde{F}$ and $\tilde{\mu}$ are replaced by $X$ and $\mu$, respectively, and $u$ is replaced by any function $u \in W^{1,2}(X)$. The result may then be viewed as a particular case of $[9$, Theorem 5.1] (where $X$ is noncompact), but we give a simpler, direct proof. We may assume that $\bar{u}=0$; otherwise replace $u$ by $u-\bar{u}$. If there were 
no such constant $c_{1}$ there would be a sequence of functions $\left(u_{n}\right) \subset W^{1,2}(X)$ such that

$$
\int_{X} u_{n} d \mu=0, \quad \int_{X} u_{n}^{2} d \mu=1, \quad \lim _{n \rightarrow \infty} \int_{X}\left|\nabla u_{n}\right|^{2} d \mu=0 .
$$

After passing to a subsequence we may assume that $u_{n}$ converges in $L^{2}(X, \mu)$ and pointwise $\mu$-a.e. in $X$ to some $u \in L^{2}(X, \mu)$ with $\int_{X}|\nabla u|^{2} d \mu=0$; this follows from $[25, \S 1.6]$, or from [9, Lemmas 9.1 and 9.2] applied to $Y=\mathbb{R}$ with $X$ replaced by any $(m-1)$-simplex $s$ of $X$; cf. [9, Corollary 9.2]. Consequently, $u$ equals a constant $c_{s} \mu$-a.e. on each $m$-simplex $s$. For two $m$-simplexes $s, s^{\prime}$ such that $s \cap s^{\prime}$ is an $(m-1)$-simplex we have $c_{s}=c_{s^{\prime}}$ in view of $\left[25\right.$, Theorem 1.12.2]. For arbitrary $m$-simplexes $s, s^{\prime}$ of the admissible polyhedron $X$ there is a chain $s_{0}, \ldots, s_{p}$ of $m$-simplexes of $X$ such that $s_{j-1} \cap s_{j}$ is an $(m-1)$-simplex of $X$ for $j \in\{1, \ldots, p\}$, and that $s_{0}=s, s_{p}=s^{\prime}$; see [18, Lemma 5]. We conclude that $c_{s}=c_{s^{\prime}}$, and so there is a constant $c$ such that $u=c \mu$-a.e. in $s$ for all $m$-simplexes $s$ of $X$. Consequently, $u_{n} \rightarrow c$ in $L^{2}(X, \mu)$, and pointwise $\mu$-a.e. in $X$. The constant $c$ equals 0 because $0=\int_{X} u_{n} d \mu \rightarrow c \mu(X)$, but on the other hand $c=1 / \sqrt{\mu(X)}$ because $1=\int_{X} u_{n}^{2} d \mu \rightarrow c^{2} \mu(X)$. This contradiction establishes (4.2).

Write

$$
E_{0}=\inf \left\{E(\tilde{\varphi} \mid \widetilde{F}): \tilde{\varphi} \in \mathcal{A}_{\tilde{\chi}}\right\},
$$

and consider a corresponding minimizing sequence $\left(\tilde{\varphi}_{i}\right) \subset \mathcal{A}_{\tilde{\chi}}$. The midpoint map of two maps $\tilde{\varphi}, \tilde{\varphi}^{\prime} \in \mathcal{A}_{\tilde{\chi}}$ is likewise of class $\mathcal{A}_{\tilde{\chi}}$. Furthermore, the distance function $d_{\widetilde{Y}}\left(\tilde{\varphi}, \tilde{\varphi}^{\prime}\right) \in W^{1,2}(\widetilde{X})$ (see for example [14, paragraph preceding (2.4)]) is essentially $\Gamma_{X}$-invariant in view of (4.1). Now apply [25, Theorem 2.2] or $[14,(3.3),(3.5)]$ (involving quadrilateral comparison) to each of the essentially $\Gamma_{X}$-invariant functions $\tilde{u}_{i j}:=d_{\widetilde{Y}}\left(\tilde{\varphi}_{i}, \tilde{\varphi}_{j}\right) \in W_{\mathrm{loc}}^{1,2}(\widetilde{X})$ considered on each $m$-simplex $\tilde{s}_{\nu}$. After summation over $\nu$, this leads to

$$
\lim _{i, j \rightarrow \infty} \int_{\widetilde{F}}\left|\nabla \tilde{u}_{i j}\right|^{2} d \tilde{\mu}=0 .
$$

Because $\tilde{u}_{i j}$ is $\Gamma_{X}$-invariant the integrals in (4.4) and in (4.5) (below) do not depend on the particular choice of $\widetilde{F}$. Denoting by $a_{i j}$ the mean value of $\tilde{u}_{i j} \mid \widetilde{F}$ with respect to $\tilde{\mu} \mid \widetilde{F}$, we obtain from (4.2) and (4.4)

$$
\lim _{i, j \rightarrow \infty} \int_{\widetilde{F}}\left(\tilde{u}_{i j}-a_{i j}\right)^{2} d \tilde{\mu}=0 .
$$


Note that $\tilde{u}_{i j}=0$ q.e. (in particular $\tilde{\mu}$-a.e.) in $\widetilde{F} \backslash \widetilde{\Omega}$, and that $\tilde{\mu}(\widetilde{F} \backslash \widetilde{\Omega})>0$ (under our temporary hypothesis that $\bar{\Omega} \neq X$ ). It follows from (4.5) (with integration over $\widetilde{F} \backslash \widetilde{\Omega}$ only) that $a_{i j} \rightarrow 0$ as $i, j \rightarrow \infty$. Inserting this in (4.5) as it stands yields

$$
\int_{\widetilde{F}} \tilde{u}_{i j}^{2} d \tilde{\mu} \leqslant 2 \int_{\widetilde{F}}\left(\tilde{u}_{i j}-a_{i j}\right)^{2} d \tilde{\mu}+2 a_{i j}^{2} \int_{\widetilde{F}} d \tilde{\mu} \rightarrow 0 \quad \text { as } i, j \rightarrow \infty
$$

since $\tilde{u}_{i j}^{2} \leqslant 2\left(\tilde{u}_{i j}-a_{i j}\right)^{2}+2 a_{i j}^{2}$. Clearly, this applies with $\widetilde{F}$ replaced by any $m$-simplex of $\widetilde{F}$, or even of $\widetilde{X}$, hence also with $\widetilde{F}$ replaced by any closed star $\widetilde{S}$ in $\widetilde{X}$. The Cauchy sequence $\left(\tilde{\varphi}_{i}\right)$ in $L^{2}(\widetilde{S}, \widetilde{Y})$ therefore converges to a map $\tilde{\varphi}_{0} \in L^{2}(\widetilde{S}, \widetilde{Y})$, i.e.,

$$
\int_{\widetilde{S}} d_{\widetilde{Y}}^{2}\left(\tilde{\varphi}_{i}, \tilde{\varphi}_{0}\right) d \tilde{\mu} \rightarrow 0 \quad \text { as } i \rightarrow \infty
$$

The sequence $\left(E\left(\tilde{\varphi}_{i}\right)\right)$ is bounded because $E\left(\tilde{\varphi}_{i} \mid \tilde{s}\right) \leqslant E\left(\tilde{\varphi}_{i} \mid \widetilde{F}\right) \rightarrow E_{0}$ for any $m$-simplex $\tilde{s}$ of $\widetilde{S}$ and any choice of a "fundamental domain" $\widetilde{F}$ containing $\tilde{s}$. It follows by Lemma 2.5 with $X$ replaced by $\widetilde{S}$ (compact and admissible) that $\tilde{\varphi}_{0} \in \mathcal{E}(\widetilde{S}, \widetilde{Y})$, and after passing to a subsequence, still denoted $\left(\tilde{\varphi}_{i}\right)$, that

$$
\frac{1}{k} \sum_{i=1}^{k} d_{\widetilde{Y}}\left(\tilde{\varphi}_{i}, \tilde{\varphi}_{0}\right) \rightarrow 0 \quad \text { q.e. in } \widetilde{S} \text { as } k \rightarrow \infty
$$

when also $\tilde{\varphi}_{0}$ is taken to be quasicontinuous in $\widetilde{S}$. As the stars $\widetilde{S}$ vary, the corresponding maps $\tilde{\varphi}_{0}$ (after being redefined on a $\mu$-nullset) become restrictions of a single quasicontinuous map $\tilde{\varphi}_{0} \in \mathcal{E}_{\text {loc }}(\widetilde{X}, \widetilde{Y})$, and (4.6) holds q.e. in $\widetilde{X}$. Since $\widetilde{X}$ has a countable base, countably many stars $\widetilde{S}$ cover $\widetilde{X}$, and (4.6) therefore holds q.e. in $\widetilde{X}$ as $k \rightarrow \infty$.

We show that $\tilde{\varphi}_{0} \in \mathcal{A}_{\tilde{\chi}}$. Because each $\tilde{\varphi}_{i} \in \mathcal{A}$ we have for $\gamma \in \Gamma_{X}$ by the triangle inequality, interpolating the point $\tilde{\varphi}_{i} \circ \gamma=\chi_{*}(\gamma) \circ \tilde{\varphi}_{i}$, summing over $i \in\{1, \ldots, k\}$, and dividing by $k$ :

$$
\begin{aligned}
d_{\widetilde{Y}}\left(\tilde{\varphi}_{0} \circ \gamma, \chi_{*}(\gamma) \circ \tilde{\varphi}_{0}\right) \leqslant & \frac{1}{k} \sum_{i=1}^{k} d_{\widetilde{Y}}\left(\tilde{\varphi}_{0} \circ \gamma, \tilde{\varphi}_{i} \circ \gamma\right) \\
& +\frac{1}{k} \sum_{i=1}^{k} d_{\widetilde{Y}}\left(\chi_{*}(\gamma) \circ \tilde{\varphi}_{i}, \chi_{*}(\gamma) \circ \tilde{\varphi}_{0}\right) \\
\rightarrow & 0 \text { q.e. in } \tilde{X} \text { as } k \rightarrow \infty,
\end{aligned}
$$


according to (4.6). Here we use that $\Gamma_{Y}$ is countable and that $\chi_{*}(\gamma) \in \Gamma_{Y}$ is an isometry of $\widetilde{Y}$. From (4.7) it follows that $d_{\widetilde{Y}}\left(\tilde{\varphi}_{0} \circ \gamma, \chi_{*}(\gamma) \circ \tilde{\varphi}_{0}\right)=0$, that is,

$$
\chi_{*}(\gamma) \circ \tilde{\varphi}_{0}=\tilde{\varphi}_{0} \circ \gamma \quad \text { q.e. in } \widetilde{X}
$$

for every $\gamma \in \Gamma_{X}$. Because $\tilde{\varphi}_{0} \in \mathcal{E}_{\widetilde{X}}(\widetilde{\widetilde{X}}, \widetilde{Y})$ we conclude from (4.8) that $\tilde{\varphi}_{0} \in \mathcal{A}$. Inserting $\tilde{\varphi}_{i}=\tilde{\chi}$ q.e. in $\widetilde{X} \backslash \widetilde{\Omega}$ in (4.6) (with $\widetilde{S}$ replaced by $\widetilde{X}$ ) we obtain $d_{\widetilde{Y}}\left(\tilde{\chi}, \tilde{\varphi}_{0}\right)=0$ q.e. in $\widetilde{X} \backslash \widetilde{\Omega}$. Thus $\tilde{\varphi}_{0}=\tilde{\chi}$ q.e. in $\widetilde{X} \backslash \widetilde{\Omega}$, and so altogether $\tilde{\varphi}_{0} \in \mathcal{A}_{\tilde{\chi}}$.

Finally, by definition of $E_{0}$,

$$
\begin{aligned}
E_{0} \leqslant E\left(\tilde{\varphi}_{0} \mid \widetilde{F}\right) & =\sum_{\nu=1}^{n} E\left(\tilde{\varphi}_{0} \mid \tilde{s}_{\nu}\right) \leqslant \sum_{\nu=1}^{n} \liminf _{i \rightarrow \infty} E\left(\tilde{\varphi}_{i} \mid \tilde{s}_{\nu}\right) \\
& \leqslant \liminf _{i \rightarrow \infty} \sum_{\nu=1}^{n} E\left(\tilde{\varphi}_{i} \mid \tilde{s}_{\nu}\right)=\lim _{i \rightarrow \infty} E\left(\tilde{\varphi}_{i} \mid \widetilde{F}\right)=E_{0},
\end{aligned}
$$

the second inequality by [25, Theorem 1.6.1] or [9, Lemma 9.1] (on lower semicontinuity of energy), applied to the $m$-simplexes $\tilde{s}_{\nu}$ of $\widetilde{F}$. Consequently, $E\left(\tilde{\varphi}_{0} \mid \widetilde{F}\right)=E_{0}$, and so $\tilde{\varphi}_{0} \in \mathcal{A}_{\tilde{\chi}}$ minimizes the energy of restrictions of all maps of class $\mathcal{A}_{\tilde{\chi}}$ to any prescribed "fundamental domain" $\widetilde{F}$ for $\Gamma_{X}$, as in the paragraph preceding the one containing (3.3).

Step 2 . The remaining properties of $\tilde{\varphi}_{0}$ and its projection $\varphi_{0}$. Fix a point $\tilde{p} \in \widetilde{\Omega} \cup \partial \widetilde{\Omega}$. By suitably subdividing the given triangulation of $X$ and hence of $\widetilde{X}$ we obtain by [37, Lemma 1, p. 144] a triangulation $T$ of $X$ in which $p:=p_{X}(\tilde{p})$ is a vertex, and by [42, Theorem 35] that every open star is contained in some ball in $X$ of radius $r_{X}$. It follows that $\tilde{p}$ is a vertex of the induced triangulation $\widetilde{T}$ of $\widetilde{X}$, and the open star $\widetilde{S}$ of $\tilde{p}$ in $(\widetilde{X}, \widetilde{T})$ is contained in $B_{\widetilde{X}}\left(\tilde{p}, r_{X}\right)$ because $B_{\widetilde{X}}\left(\tilde{p}, r_{X}\right)$ projects isometrically on $B_{X}\left(p, r_{X}\right)$ (cf. (2.2)) and hence $p_{X}(\widetilde{S})=S$. It follows that $\widetilde{S} \subset \widetilde{F}$ for a suitable "fundamental domain" $\widetilde{F}$ in $(\widetilde{X}, \widetilde{T})$. According to $[18$, Theorem 1 and Corollary 3], $\widetilde{S}$ is admissible, along with $\widetilde{X}$. Furthermore, $\widetilde{S}$ satisfies the Poincaré inequality

$$
\int_{\widetilde{S}} \tilde{u}^{2} d \tilde{\mu} \leqslant c_{2} \int_{\widetilde{S}}|\nabla \tilde{u}|^{2} d \tilde{\mu} \quad \text { for all } \tilde{u} \in W_{0}^{1,2}(\widetilde{S}),
$$

for some constant $c_{2}$; see [9, Remark 5.5, p. 76] or [13, Lemma 1(c)]. In a triangulation of $\widetilde{S}$ denote by $\tilde{\omega} \Subset \widetilde{S}$ an open star containing $\tilde{p}$. (Here $\tilde{\omega}$ replaces the disc $D$ in $[28$, p. 63$]$, or the star $B^{j}$ in the paragraph containing 
(3.6) above, both for the free homotopy problem.) Then $\tilde{F} \cap \gamma(\tilde{\omega})=\varnothing$ for all $\gamma \in \Gamma_{X} \backslash\{$ id $\}$, cf. (2.2) and subsequent text. Given a (quasicontinuous) $\operatorname{map}_{\widetilde{\Omega}} \tilde{\theta} \in \mathcal{E}(\widetilde{S}, \widetilde{Y})$ such that $\tilde{\theta}=\tilde{\varphi}_{0}$ in $\widetilde{S} \backslash(\tilde{\omega} \cap \widetilde{\Omega})$ (that is, in $\widetilde{S} \backslash \tilde{\omega}$ and in $\widetilde{S} \backslash \widetilde{\Omega})$, define $\tilde{\theta}^{\prime}: \widetilde{X} \rightarrow \widetilde{Y}$ in analogy with (3.6) by

$$
\tilde{\theta}^{\prime}= \begin{cases}\chi_{*}(\gamma) \circ \tilde{\theta} \circ \gamma^{-1} & \text { in } \gamma(\tilde{\omega}) \text { for any } \gamma \in \Gamma_{X} \\ \tilde{\varphi}_{0} & \text { elsewhere in } \tilde{X} .\end{cases}
$$

In particular, $\tilde{\theta}^{\prime}=\tilde{\varphi}_{0}$ in $\widetilde{F} \backslash \widetilde{S}$ because $\widetilde{F}$ is "almost fundamental" and hence $\widetilde{F} \backslash \widetilde{S} \subset \widetilde{F} \backslash \tilde{\omega}=\widetilde{F} \backslash \bigcup_{\gamma \in \Gamma_{X}} \gamma(\tilde{\omega})$. For every $\gamma \in \Gamma_{X}$ we have

$$
\tilde{\theta}^{\prime}=\chi_{*}(\gamma) \circ \tilde{\theta} \circ \gamma^{-1} \quad \text { q.e. in } \gamma(\widetilde{S}) .
$$

Indeed, this holds (even everywhere) in $\gamma(\tilde{\omega})$ by the former equation of (4.10). And (4.11) also holds q.e. in $\gamma(\widetilde{S} \backslash \tilde{\omega})$ because we have, q.e. in that set,

$$
\tilde{\theta}^{\prime}=\tilde{\varphi}_{0}=\chi_{*}(\gamma) \circ \tilde{\varphi}_{0} \circ \gamma^{-1}=\chi_{*}(\gamma) \circ \tilde{\theta} \circ \gamma^{-1}
$$

according to the latter equation of (4.10) followed by (4.8), and next by $\tilde{\varphi}_{0}=\tilde{\theta}$ in $\widetilde{S} \backslash \tilde{\omega}$, whence $\tilde{\varphi}_{0} \circ \gamma^{-1}=\tilde{\theta} \circ \gamma^{-1}$ in $\gamma(\widetilde{S} \backslash \tilde{\omega})$. Explicitly, the latter equation of (4.10) reads

$$
\tilde{\theta}^{\prime}=\tilde{\varphi}_{0} \quad \text { in } \widetilde{R}:=\widetilde{X} \backslash \bigcup_{\gamma \in \Gamma_{X}} \gamma(\tilde{\omega}) .
$$

The sets $\gamma(\widetilde{S})\left(\gamma \in \Gamma_{X}\right)$ together with the interior $\widetilde{R}^{\circ}$ of $\widetilde{R}$ form a countable open cover of $\widetilde{X}$ because $\tilde{\omega} \Subset \widetilde{S}$. From (4.11) and (4.12) it follows that $\tilde{\theta}^{\prime}$ is quasicontinuous and of locally finite energy in $\widetilde{X}$ because $\tilde{\varphi}_{0}$ and each $\chi_{*}(\gamma) \circ$ $\tilde{\theta} \circ \gamma^{-1}$ have these two properties in $\widetilde{R}^{\circ} \subset \widetilde{X}$ and in $\gamma(\widetilde{S})$, respectively, cf. (i) preceding Equation (2.21). Similarly, $\tilde{\theta}^{\prime}=\tilde{\varphi}_{0}=\tilde{\chi}$ q.e. in $\widetilde{X} \backslash \widetilde{\Omega}$. Altogether, this shows that $\tilde{\theta}^{\prime} \in \mathcal{A}_{\tilde{\chi}}$.

In what follows we assume for convenience that $\tilde{\omega} \cap \widetilde{\Omega}$ is connected (otherwise consider each component of $\tilde{\omega} \cap \widetilde{\Omega}$ separately). In the display (4.13) below, the first equation holds because $\tilde{\theta}=\tilde{\theta}^{\prime}$ in $\tilde{\omega}$ by (4.10) with $\gamma=$ id. The second equation in (4.13) holds by Lemma 2.3(b) (applied with $\widetilde{X}$ replaced by $\widetilde{F}$, or rather by each $m$-simplex of $\widetilde{F})$ because $\tilde{\mu}(\partial \widetilde{S})=0$. The inequality $E\left(\tilde{\theta}^{\prime} \mid \widetilde{F}\right) \geqslant E\left(\tilde{\varphi}_{0} \mid \widetilde{F}\right)$ holds because $\tilde{\varphi}_{0}, \tilde{\theta}^{\prime} \in \mathcal{A}_{\tilde{\chi}}$ and that $\tilde{\varphi}_{0}$ is energy minimizing relative to $\mathcal{A}_{\tilde{\chi}}$; see the end of Step 1. Again by Lemma 2.3(b) we similarly have $e\left(\tilde{\theta^{\prime}} \mid \widetilde{F}\right)=e\left(\tilde{\varphi}_{0} \mid \widetilde{F}\right) \mu$-a.e. in $\widetilde{F} \backslash \widetilde{S}$ because $\tilde{\theta}^{\prime}=\tilde{\varphi}_{0}$ in $\widetilde{F} \backslash \widetilde{S}$, 
as noted after (4.10). Altogether we thus obtain

$$
\begin{aligned}
E(\tilde{\theta})=E\left(\tilde{\theta}^{\prime} \mid \widetilde{S}\right) & =E\left(\tilde{\theta}^{\prime} \mid \widetilde{F}\right)-\int_{\widetilde{F} \backslash \widetilde{S}} e\left(\tilde{\theta}^{\prime} \mid \widetilde{F}\right) d \tilde{\mu} \\
& \geqslant E\left(\tilde{\varphi}_{0} \mid \widetilde{F}\right)-\int_{\widetilde{F} \backslash \widetilde{S}} e\left(\tilde{\varphi}_{0} \mid \widetilde{F}\right) d \tilde{\mu}=E\left(\tilde{\varphi}_{0} \mid \widetilde{S}\right)
\end{aligned}
$$

again because $\tilde{\mu}(\partial \widetilde{S})=0$. In view of the property of $\tilde{\theta}$ imposed in the lines preceding (4.10) we conclude that $\tilde{\varphi}_{0} \mid \widetilde{S}$ is the unique variational solution to the ordinary Dirichlet problem in $\widetilde{S}$, minimizing the energy of maps $\tilde{\theta}$ of class

$$
\mathcal{E}_{\tilde{\chi} \mid \widetilde{S}}(\tilde{\omega} \cap \widetilde{\Omega}, \widetilde{Y}):=\{\tilde{\theta} \in \mathcal{E}(\widetilde{S}, \widetilde{Y}): \tilde{\theta}=\tilde{\chi} \text { in } \widetilde{S} \backslash(\tilde{\omega} \cap \widetilde{\Omega})\} ;
$$

cf. [14, Theorem 1], applied with $X, Y, \Omega, \varphi, \chi \operatorname{replaced}$ by $\widetilde{S}, \widetilde{Y}, \tilde{\omega} \cap \widetilde{\Omega}, \tilde{\varphi}_{0} \mid \widetilde{S}$, $\tilde{\chi} \mid \widetilde{S}$, noting that $\widetilde{S}$ is admissible and satisfies the Poincaré inequality (4.9), and that $\tilde{\omega} \cap \widetilde{\Omega} \Subset \widetilde{S}$.

Every component of $\tilde{\omega} \cap \widetilde{\Omega}$ is regular. We shall, however, only need that every point $\tilde{x} \in \tilde{\omega} \cap \partial \widetilde{\Omega}$ is regular for $\tilde{\omega} \cap \widetilde{\Omega}$, that is, $\widetilde{X} \backslash(\tilde{\omega} \cap \widetilde{\Omega})$ is non-thin at $\tilde{x}$; and that holds because even $\tilde{X} \backslash \widetilde{\Omega}$ is non-thin at $\tilde{x}$, noting that $X \backslash \Omega$ is non-thin at $x:=p_{X}(\tilde{x})$ because $\Omega$ is regular, that $p_{X}$ is a local isometry, and that thinness is a local property.

We have chosen $\tilde{p} \in \widetilde{\Omega} \cup \partial \widetilde{\Omega}$. Suppose first that $\tilde{p} \in \partial \widetilde{\Omega}$. Because we have seen that the points of $\tilde{\omega} \cap \partial \widetilde{\Omega}$ are regular for $\tilde{\omega} \cap \widetilde{\Omega}$, and because $\tilde{\chi}$ is continuous, it follows from [14, Theorem 1] or [16, Theorem 4] (applied as above) that a version of $\tilde{\varphi}_{0}$ is continuous up to the boundary at the portion $\tilde{\omega} \cap \partial \widetilde{\Omega} \ni \tilde{p}$ of the boundary of $\tilde{\omega} \cap \widetilde{\Omega}$, and hence up to the entire boundary $\partial \widetilde{\Omega}$, by varying $\tilde{p}$ (and $\tilde{\omega})$.

If instead $\tilde{p} \in \widetilde{\Omega}$, and hence $\tilde{p} \in \widetilde{S} \cap \widetilde{\Omega}$, choose the open star $\tilde{\omega} \ni \tilde{p}$, now in a triangulation of $\widetilde{S} \cap \widetilde{\Omega}$, and so that $\tilde{\omega} \Subset \widetilde{S} \cap \widetilde{\Omega}$. Then $\tilde{\varphi}_{0}$ is, in particular, locally energy minimizing in $\tilde{\omega} \cap \widetilde{\Omega}=\tilde{\omega}$ (cf. the paragraph preceding the one containing (3.6)). It follows by [11, Theorem 1] or [17, Theorem 1] (applied as above, noting that now $\widetilde{\Omega} \cap \tilde{\omega}=\tilde{\omega}$ ) that a version of $\tilde{\varphi}_{0}$ is (locally uniformly) Hölder continuous in $\tilde{\omega}$, and hence in $\widetilde{\Omega}$, by varying $\tilde{p}$.

Because $\tilde{\varphi}_{0}=\tilde{\chi}$ q.e. off $\widetilde{\Omega}$, and $\tilde{\chi}$ is continuous, we altogether conclude that $\tilde{\varphi}_{0}\left(\in \mathcal{A}_{\tilde{\chi}}\right)$ has a continuous version satisfying $(3.2)$ and $(4.1)$ everywhere in $\tilde{X}$ (with $\tilde{\varphi}$ replaced by $\tilde{\varphi}_{0}$ ), so $\tilde{\varphi}_{0}$ is $\chi_{*}$-equivariant. It follows that $\tilde{\varphi}_{0}$ has a continuous projection $\varphi_{0}: X \rightarrow Y$, freely homotopic with $\chi$, as shown in the paragraph containing (3.2). The homotopy $\Phi$ constructed there has $\Phi(x, t)=\chi(x)$ for $x \in X \backslash \Omega$, and $\varphi_{0}$ is therefore homotopic with $\chi$ through maps of class $C_{\chi}(\Omega, Y)$, so $\varphi_{0} \in \mathcal{H}_{\chi}(\Omega, Y)$. 
To show that $E\left(\varphi_{0}\right) \leqslant E(\varphi)$ for every $\varphi \in \mathcal{H}_{\chi}(\Omega, Y)$, note first that $E\left(\tilde{\varphi}_{0} \mid \widetilde{F}\right) \leqslant E(\tilde{\varphi} \mid \widetilde{F})$ by $(4.3)$ for any $\tilde{\varphi} \in \mathcal{A}_{\tilde{\chi}}$ (see the end of Step 1). For continuous $\tilde{\varphi} \in \mathcal{A}_{\tilde{\chi}}$ we have shown in (3.3) that $E(\tilde{\varphi} \mid \widetilde{F})=E(\varphi)$, where now $\varphi \in \mathcal{H}_{\chi}(\Omega, Y)$ denotes the projection of $\tilde{\varphi}$ (see the paragraph containing (3.2)). As shown in the beginning of Step 1, every map $\varphi \in \mathcal{H}_{\chi}(\Omega, Y)$ has a $\chi_{*}$-equivariant lift $\tilde{\varphi}$ which equals $\tilde{\chi}$ in $\widetilde{X} \backslash \widetilde{\Omega}$, and so $\tilde{\varphi} \in \mathcal{A}_{\tilde{\chi}}$. Consequently, $E\left(\varphi_{0}\right)=E\left(\tilde{\varphi}_{0} \mid \widetilde{F}\right) \leqslant E(\tilde{\varphi} \mid \widetilde{F})=E(\varphi)$, so $\varphi_{0}$ minimizes energy within $\mathcal{H}_{\chi}(\Omega, Y)$. Furthermore, $\varphi_{0}$ is (locally uniformly) Hölder continuous in $\Omega$ because $\tilde{\varphi}_{0}$ is so in $\widetilde{\Omega}$ and because $p_{X}$ and $p_{Y}$ are local isometries.

The proof that $\varphi_{0}$ is harmonic in $\Omega$ (cf. Remark 2.6) is the same as in Step 4 of the proof of Theorem 3.2, except that $X, \varphi$ shall now be replaced by $\Omega, \varphi_{0}$, and any subset of $X$ by its intersection with $\Omega$ (though the homotopy $\Psi$ is still defined on $X \times[0,1]$, and the set $X \backslash \bar{U}$ shall remain unaltered).

Step 3. Uniqueness. Consider two solutions $\varphi_{0}, \varphi_{1}$ to the Dirichlet homotopy problem, that is, $\varphi_{0}, \varphi_{1} \in \mathcal{H}_{\chi}(\Omega, Y)$ have (the same) minimal energy. Then $\varphi_{0}, \varphi_{1}$ lift to continuous energy minimizing $\chi_{*}$-equivariant maps $\tilde{\varphi}_{0}, \tilde{\varphi}_{1}$. Step 5 of the proof of Theorem 3.2 carries over (with $\mathcal{H}$ replaced by $\mathcal{H}_{\chi}(\Omega, Y)$ ). Thus $d_{\widetilde{Y}}\left(\tilde{\varphi}_{0}, \tilde{\varphi}_{1}\right)$ is a constant $\tilde{d}$, which equals 0 because $\tilde{\varphi}_{0}=\tilde{\varphi}_{1}$ on $\widetilde{X} \backslash \widetilde{\Omega} \neq \varnothing$. Consequently, $\tilde{\varphi}_{0}=\tilde{\varphi}_{1}$, and so indeed $\varphi_{0}=\varphi_{1}$, by projection. Thus $\varphi=\varphi_{0}$ is the unique solution to our Dirichlet homotopy problem for the present case $\bar{\Omega} \neq X$.

Step 4. The general case $\overline{\Omega \cap b X} \neq b X$, in particular $b X \neq \varnothing$. Here $\Omega \subset X \backslash \sigma$ for some nonvoid relatively open subset $\sigma$ of $b X$. By subdivision of the triangulation of $X$ we arrange that $\sigma$ contains an $(m-1)$-simplex $\tau$ of $b X$. (To justify this, write $\sigma=U \cap b X$ for some open subset $U$ of $X$. Choose a point $a \in \sigma$ and an open subset $V$ of $X \backslash\{a\}$ so that $U \cup V=X$. According to [42, Theorem 35] there exists a subdivision of the triangulation of $X$ such that an open star $S$ containing $a$ is a subset of $U$, the alternative $S \subset V$ being ruled out since $a \notin V$. Because $a \in b X$, there exists an $(m-1)$ simplex $\tau$ of $S$ contained in $b X$, and hence $\tau \subset U \cap b X=\sigma$.)

Consider the disjoint union $X^{*}$ of $X$ and another copy $X^{\prime}$ of $X$, though glued together along $\tau$. Then $X^{*}$ is a compact admissible Riemannian polyhedron (possibly with boundary), and $X$ and $X^{\prime}$ are compact subpolyhedra of $X^{*}{ }^{7}$ Denote by $\iota: X^{*} \rightarrow X^{*}$ the natural involution of $X^{*}$, interchanging $X$ and $X^{\prime}$. For any map $\varphi \in \mathcal{H}_{\chi}(\Omega, Y)$ denote $\varphi^{*}$ the extension of

${ }^{7}$ For the easy proof that $X^{*}$ is admissible, apply $[18$, Lemma 5$]$ as in $[13$, p. 773 , third and fourth paragraphs]. Here $Z_{+}$and $Z_{-}$correspond to the present $X$ and its stated copy, and hence $Z$ is the present $X^{*}$; furthermore, $Z_{0}:=Z_{+} \cap \tau=Z_{-} \cap \tau$ corresponds to the present $\tau$. 
$\varphi$ by $\chi \circ \iota \mid X^{*} \backslash X$; then $\varphi^{*} \in \mathcal{E}\left(X^{*}, Y\right)$ and $E\left(\varphi^{*}\right)=E(\varphi)+E\left(\chi \circ \iota \mid X^{\prime}\right)=$ $E(\varphi)+E(\chi)$; cf. [9, Theorem 9.1 and Remark 9.1]. In particular, $\chi^{*} \in$ $\mathcal{E}\left(X^{*}, Y\right)$ is the symmetric extension of $\chi$ to $X^{*}$, and clearly $\varphi^{*} \in \mathcal{H}_{\chi^{*}}(\Omega, Y)$. Conversely, for any $\varphi^{*} \in \mathcal{H}_{\chi^{*}}(\Omega, Y)$, the restriction $\varphi:=\varphi^{*} \mid X$ is of class $\mathcal{H}_{\chi}(\Omega, Y)$. Furthermore, $\varphi$ is then an energy minimizer relative to $\mathcal{H}_{\chi}(\Omega, Y)$ if and only if $\varphi^{*}$ is the unique energy minimizer $\varphi_{0}^{*}$ relative to $\mathcal{H}_{\chi^{*}}(\Omega, Y)$. Note at this point that $\Omega \subset X$ is a connected regular open subset also of $X^{*}$, but $\Omega$ is not dense in $X^{*}$; thus Theorem 4.1 applies with $X, \chi$ replaced by $X^{*}, \chi^{*}$. We conclude that the restriction $\varphi_{0}$ of $\varphi_{0}^{*}$ to $X$ is the unique energy minimizer relative to $\mathcal{H}_{\chi}(\Omega, Y)$, and $\varphi_{0}\left|\Omega=\varphi_{0}^{*}\right| \Omega$ is indeed harmonic and (locally uniformly) Hölder continuous, by Theorem 4.1 applied to $X^{*}, \chi^{*}, \Omega$.

\section{The case of Riemannian manifold targets}

In this final section we specialize the target by replacing the metric space $\left(Y, d_{Y}\right)$ with a (smooth) Riemannian manifold $(Y, h)$ without boundary (in particular, $Y$ is locally compact and carries an intrinsic Riemannian distance $d_{Y}$ associated with the Riemannian metric $h$ ). On the other hand, we no longer require that the Riemannian metric $g$ on the compact admissible $m$-dimensional source polyhedron $X$ be simplex-wise smooth, but merely measurable, again with elliptic bounds $\Lambda_{X}, \Lambda_{X}^{-1}$, that is,

$$
\Lambda_{X}^{-2} \leqslant \sum_{i, j=1}^{m} g_{i j}(x) \xi^{i} \xi^{j}=\sum_{i, j=1}^{m} g^{i j}(x) \xi_{i} \xi_{j} \leqslant \Lambda_{X}^{2}
$$

for a.e. $x \in X$ and for every $m$-tuple $\left(\xi^{1}, \ldots, \xi^{m}\right)$, respectively $\left(\xi_{1}, \ldots, \xi_{m}\right)$, in $\mathbb{R}^{m}$ with Euclidean norm $|\xi|=1$. Measurability and the term "almost everywhere (a.e.)" refer to a fixed Riemannian volume measure on $X$, for example the measure $\mu^{e}$ corresponding to the Euclidean Riemannian metric $g^{e}$ on $X$ (cf. [9, p. 52]). We then dispose of an alternative concept of energy, of a more explicit character and defined for example in terms of an isometric $C^{1}$-embedding of $(Y, h)$ as a Riemannian submanifold of some $\mathbb{R}^{N}$, as in Nash's theorem: A map $\varphi: X \rightarrow Y$ is said to be of class $\mathcal{E}(X, Y)$ if the components $\varphi^{1}, \ldots, \varphi^{N}$ of $\varphi: X \rightarrow Y \hookrightarrow \mathbb{R}^{N}$ are of class $W^{1,2}(X)$. In the affirmative case the energy density of $\varphi$ is defined by

$$
e(\varphi)=\sum_{\nu=1}^{N}\left|\nabla \varphi^{\nu}\right|^{2}=\sum_{\nu=1}^{N} g^{i j} \partial_{i} \varphi^{\nu} \partial_{j} \varphi^{\nu} \in L^{1}(X, \mu)
$$


(using the standard summation convention); and the energy is defined by $E(\varphi)=\int_{X} e(\varphi) d \mu(<\infty)$. Here $\mu$ denotes the Riemannian volume measure on $X$ corresponding to $g$, that is, $d \mu=\sqrt{\operatorname{det} g} d \mu^{e}$ in terms of the covariant components $g_{i j}(i, j=1, \ldots, m)$ of $g$ and their determinant. For an equivalent, covariant definition of $E(\varphi)$, see [9, Definition 9.2 and Lemma 9.3]. If $g$ is simplex-wise smooth and if $\varphi(X)$ has compact closure in $Y$, then the energy density $e(\varphi)$ from (5.1) equals a.e. the energy density $e(\varphi)$ defined by (2.21) (i.e., as the weak limit of the $\varepsilon$-approximate energy density $e_{\varepsilon}(\varphi)$ from (2.18) as $\varepsilon \rightarrow 0)$, provided that $E(\varphi)<\infty$ in the one sense or the other [9, Theorem 9.2(b)]. The hypothesis $\varphi(X) \Subset Y$ is of course fulfilled if $Y$ is compact, or if $X$ is compact and $\varphi$ is continuous. Every map $X \rightarrow Y$ of finite energy in the present sense has a quasicontinuous version (because every function of class $W^{1,2}(X)$ has so $[9$, p. $\left.106 \mathrm{f}]\right)$.

Recall that a complete Riemannian manifold $(Y, h)$ with intrinsic Riemannian distance function $d_{Y}$ is a geodesic space, and $\left(Y, d_{Y}\right)$ has nonpositive Alexandrov curvature if and only if $(Y, h)$ has nonpositive sectional curvature [2, p. 173].

Propositions 2.1 and 2.2, as well as Lemma 2.4, do not involve energy of maps, and hence carry over right away to the present setting without requiring that the Riemannian metric $g$ on $X$ be simplex-wise smooth. For the proof of Lemma 2.5 with the present energy concept (5.1), use [9, Remark 9.6, p. $165 \mathrm{f}]$ concerning lower semicontinuity of energy.

The proof of Lemma 2.7 (the three equivalent definitions of harmonicity of a map) reduces to the case where $Y$ is simply connected, in the same way as described in the latter part of the proof of the lemma. For the simply connected case, discussed in the former part of the proof, the energy convexity property in the present setting can be found in [13, Lemma 3 and eq. (10.1)]; furthermore, one shall replace [13, Theorem 1] by [13, Theorem 4] in order to establish the present version of Lemma 2.7 above. (Similarly, in footnote 5 to Remark 2.8, the reference to [13, Theorem 3] shall be replaced by [13, Theorem 5] in order to recover Remark 2.8 in the present setting.)

For Proposition 3.1 one begins by using [9, Proposition 12.1] to recover [9, Lemma 10.2(b)]. Next, use [11, Theorem 3 and Corollary 1], the latter about the weak Poincaré inequality (see $[9$, Remark 9.6 (p. 165)]), leading to (3.1), from where the above proof of Proposition 3.1 carries over to the present setting.

Finally, note that Lemma 2.3 now becomes obvious, and that Lemma 2.3 (b) remains valid for measurable $A$, even if $\mu(\partial A)>0$, because this reduces to the case $Y=\mathbb{R}$ of functions of class $W_{\text {loc }}^{1,2}(X)$ in view of (5.1); cf. [9, Remark 5.2, p. 65]. For that reason the hypothesis $\mu(\partial \Omega)=0$ in 
Theorem 4.1 can be omitted in the present setting of maps into a Riemannian manifold, see Theorem 5.2 below.

Proceeding to the solution of our two homotopy problems in the present setting of manifold target, we shall need the following lemma about approximating a measurable Riemannian metric on $X$ by simplex-wise smooth ones:

Lemma 5.1. Let $X$ be a compact admissible polyhedron, and let $g$ be a measurable Riemannian metric on $X$ with elliptic bounds $\Lambda_{X}, \Lambda_{X}^{-1}$. Consider a sequence $\left(g_{n}\right)$ of simplex-wise smooth Riemannian metrics on $X$ such that $g_{n} \rightarrow g$ pointwise a.e. as $n \rightarrow \infty$, and furthermore that $\Lambda_{X}, \Lambda_{X}^{-1}$ are elliptic bounds for each $g_{n}$ as well. For any continuous map $\varphi \in \mathcal{E}(X, Y)$ (in the present sense) and any function $f \in C(X)$ we then have

$$
\int_{X} f e(\varphi) d \mu=\lim _{n \rightarrow \infty} \int_{X} f e_{n}(\varphi) d \mu
$$

where $e_{n}(\varphi)$ denotes the energy density of $\varphi$ relative to $g_{n}$, cf. (5.1); and $\mu_{n}$ denotes the volume measure on $X$ relative to $g_{n}$; that is, $d \mu_{n}=\sqrt{\operatorname{det} g_{n}} d \mu^{e}=$ $\sqrt{\operatorname{det} g_{n} / \operatorname{det} g} d \mu$.

Proof. A sequence $\left(g_{n}\right)$ as in Lemma 5.1 is constructed in [13, p. 791, last paragraph]. In view of (5.1),

$$
\begin{aligned}
\int_{X} f e_{n}(\varphi) d \mu_{n} & =\sum_{\nu=1}^{N} \int_{X} f g_{n}^{i j} \partial_{i} \varphi^{\nu} \partial_{j} \varphi^{\nu} \sqrt{\operatorname{det} g_{n} / \operatorname{det} g} d \mu \\
& \rightarrow \sum_{\nu=1}^{N} \int_{X} f g^{i j} \partial_{i} \varphi^{\nu} \partial_{j} \varphi^{\nu} d \mu=\int_{X} f e(\varphi) d \mu
\end{aligned}
$$

as $n \rightarrow \infty$. By dominated convergence we have in fact (when $f \geqslant 0$ )

$$
f \sum_{\nu=1}^{N} g_{n}^{i j} \partial_{i} \varphi^{\nu} \partial_{j} \varphi^{\nu} \leqslant \Lambda_{X}^{2} f \sum_{\nu=1}^{N} \sum_{i=1}^{m}\left|\partial_{i} \varphi^{\nu}\right|^{2} \in L^{1}(X, \mu),
$$

and $\sqrt{\operatorname{det} g_{n}} \leqslant \Lambda_{X}^{m}, \sqrt{\operatorname{det} g} \geqslant \Lambda_{X}^{-m}$.

For the proof of Theorem 3.2 in the present setting of manifold target, refer in Step 2 for energy convexity to [13, Lemma 3] instead of [9, (11.2); 25, $2.2 \mathrm{iv}]$; and to [14, Theorem 3] instead of [14, Theorem 1]. Inserting $\tau=\frac{1}{2}$ in the last estimate in (3.23) leads after projection to Hölder equicontinuity 
of the $\varphi_{i}$ :

$$
d_{Y}\left(\varphi_{i}(x), \varphi_{i}(y)\right) \leqslant C_{10} \sqrt{E\left(\psi_{i}\right)} d_{X}^{\alpha}(x, y) \quad \text { for } x, y \in X
$$

(with $C_{10}=2 \sqrt{C_{7}}$ ), first relative to $g_{n}$ ( ative to the given measurable Riemannian metric $g$ in view of Lemma 3.1, noting that $C_{10}$ is independent of $n$, by Remark 3.4.

In Step 3, again first for $g_{n}$ and next for $g$, form in (3.31) the sum over the $m$-simplexes $s$ of $X$ and over $j \in\{1, \ldots, k\}$, and insert the resulting inequality in (3.26). Likewise, insert the former estimate (3.19) in the remainder term in (3.26). Together, this leads to

$$
\begin{aligned}
(1-\tau) E\left(\varphi_{i}\right) \leqslant & E\left(\psi_{i}\right)+C_{11} \sqrt{E\left(\psi_{i}\right)} \sum_{j=1}^{k} \sqrt{E\left(\psi_{i}\right)-E\left(w_{i}^{j}\right)} \\
& +C_{5}^{2} C_{9} \tau^{-1} E\left(\psi_{i}\right)^{m /(m+2 \alpha)}\left(E\left(\psi_{i}\right)-E\left(w_{i}^{j l}\right)\right)^{2 \alpha /(m+2 \alpha)}
\end{aligned}
$$

(where $C_{11}$ equals twice the number of $m$-simplexes in $X$ ). The midpoint maps $\tilde{w}_{i}^{j}=\frac{1}{2} \tilde{\varphi}_{i}^{j}+\frac{1}{2} \tilde{\psi}_{i}$ and $\tilde{w}_{i}^{j l}=\frac{1}{2} \tilde{\varphi}_{i}^{j}+\frac{1}{2} \tilde{\varphi}_{i}^{l}$ being $\chi_{*}$-equivariant (along with $\tilde{\varphi}_{i}^{j}$ and $\tilde{\psi}_{i}$, respectively $\tilde{\varphi}_{i}^{l}$ ), their projections $w_{i}^{j}$ and $w_{i}^{j l}$ are of class $\mathcal{H}$, and of energy $\geqslant E_{0}$. Inserting this in (5.3) and making $i \rightarrow \infty$, whereby $E\left(\psi_{i}\right)-$ $E_{0} \rightarrow 0$, we recover (3.32) and conclude that the sequence $\left(\tilde{\varphi}_{i}^{j}\right)$ indeed is minimizing. Step 4 (harmonicity) carries over right away to the present setting, using [9, Remark 9.6] in place of Lemma 9.1 there.

In the proof of Theorem 4.1 (in the fifth paragraph of Step 1) the inequalities $e(\varphi) \circ p_{X} \leqslant e(\tilde{\varphi})$ and hence $E(\varphi) \leqslant E(\tilde{\varphi} \mid \widetilde{F})$, etc., clearly remain in force in the present setting, with equality if $\tilde{\varphi}$ is continuous. At the end of Step 1, [9, Lemma 9.1] (lower semicontinuity of energy) carries over in view of $[9$, Remark 9.6]. In the case $\tilde{p} \in \partial \widetilde{\Omega}$ replace $[14$, Theorem 1$]$ and $[16$, Theorem 4$]$ by $[14$, Theorem 3]. In the case $\tilde{p} \in \widetilde{\Omega}$ replace [11, Theorem 1] and [17, Theorem 1] by [14, Theorem 3]. The rest of the proof of Theorem 4.1 carries over right away, again in view of [9, Lemma 9.1] (lower semicontinuity) and [13, Lemma 3] (energy convexity).

By way of summary, we have obtained the following combined version of Theorems 3.2 and 4.1 in the present setting of a manifold codomain, whereby $g$ is no longer required to be simplex-wise smooth:

Theorem 5.2. Suppose that the admissible Riemannian polyhedron $X$ is compact and has measurable Riemannian metric $g$ with elliptic bounds. 
Furthermore, suppose that the smooth Riemannian manifold $(Y, h)$ without boundary is compact and has nonpositive sectional curvature. The conclusions of Theorems 3.2 and 4.1 then hold true in terms of the energy concept (5.1).

\section{Acknowledgment}

The author is indebted to the referee for suggesting that, by the proof of Theorem 3.2, the results of Korevaar and Schoen [26] on the existence of energy minimizing equivariant maps (under suitable conditions on the isometric action on the target space), in particular Theorems 2.2.1 and 2.3.1 of [26], extend when the underlying compact Riemannian manifold is replaced by any compact admissible Riemannian polyhedron.

\section{References}

[1] D.H. Armitage, and S.J. Gardiner, Classical potential theory, Springer, New York, 2001.

[2] M.R. Bridson and A. Haefliger, Metric spaces of non-positive curvature, Grundlehren, 319, Springer, Berlin, 1999.

[3] M. Biroli and U. Mosco, A Saint-Venant type principle for Dirichlet forms on discontinuous media, Ann. Mat. Pura Appl. (4) 169 (1995), $125-181$.

[4] M. Brelot, Lectures on potential theory, Tata Institute of Fundamental Research, Bombay, 1960.

[5] C. Constantinescu and A. Cornea, Potential theory on harmonic spaces, Grundlehren, 158, Springer, Berlin, 1972.

[6] J.-Y. Chen, On energy minimizing mappings between and into singular spaces, Duke Math. J. 79 (1995), 77-99.

[7] J. Deny, Théorie de la capacité dans les espaces fonctionnels, Sém. Théorie du Potentiel, Paris, $9^{\mathrm{e}}$ année, $\mathrm{n}^{\circ}$ 1, 1964-1965.

[8] G. Daskalopoulos and C. Mese, Harmonic maps from 2-complexes, Commun. Anal. Geom. 14 (2006), 497-549.

[9] J. Eells, and B. Fuglede, Harmonic maps between riemannian polyhedra, Cambridge Tracts in Mathematics, 142, Cambridge University Press, 2001. 
[10] J. Eells, and J.H. Sampson, Harmonic mappings of Riemannian manifolds, Amer. J. Math. 86 (1964), 109-160.

[11] B. Fuglede, Hölder continuity of harmonic maps from Riemannian polyhedra to spaces of upper bounded curvature, Calc. Var. Partial Differential Equations 16 (2003) 375-403.

[12] - Finite energy maps from Riemannian polyhedra to metric spaces, Ann. Acad. Sci. Fenn. Math. 28 (2003), 433-458.

[13] - The Dirichlet problem for harmonic maps from Riemannian polyhedra to spaces of upper bounded curvature, Trans. Amer. Math. Soc. 357 (2005), 757-792.

[14] - Dirichlet problems for harmonic maps from regular domains, Proc. London Math. Soc. 91 (2005), 249-272.

[15] - Harmonic maps from Riemannian polyhedra to spaces of nonpositive curvature, New Trends in Potential Theory. Conference Proceedings, Bucharest, September 2002 and 2003, Theta, 2005, pp. 29-46.

[16] - A sharpening of a theorem of Bouligand. With an application to harmonic maps, Ann. Acad. Sci. Fenn. Math. 31 (2006), 173-190.

[17] - Harmonic maps from Riemannian polyhedra to geodesic spaces with curvature bounded from above, Calc. Var. Partial Differential Equations 31 (2008), 99-136.

[18] — Polyhedra as domains of harmonic maps, Manuscript.

[19] M. Gromov and R. Schoen, Harmonic maps into singular spaces and p-adic superrigidity for lattices in groups of rank one, Publ. IHES 76 (1992), 165-246.

[20] R.S. Hamilton, Harmonic maps of manifolds with boundary, Lect. Notes Math., 471, Springer, Berlin, 1975.

[21] P. Hartman, On homotopic harmonic maps, Canad. J. Math 19 (1967), $673-687$.

[22] A. Hatcher, Algebraic topology, Cambridge University Press, Cambridge, 2002.

[23] J. Jost, Generalized Dirichlet forms and harmonic maps, Calc. Var. P.D.E. 5, (1997) 1-19.

[24] A. Kasue, Convergence of Riemannian manifolds and Laplace operators II, Manuscript, 2004. 
[25] N.J. Korevaar and R.M. Schoen, Sobolev spaces and harmonic maps for metric space targets, Commun. Anal. Geom. 1 (1993), 561-659. Reprinted as Chapter X in 'Lectures on Harmonic Maps', eds. R. Schoen and S.T. Yau, Conf. Proc. and Lecture Notes in Geometry and Topology, Vol. II, 1997, 204-310.

[26] - Global existence theorems for harmonic maps to non-locally compact spaces, Commun. Anal. Geom. 5 (1997) 333-387.

[27] L. Lemaire, On the existence of harmonic maps, Thesis, Warwick University, 1977.

[28] - Applications harmoniques de surfaces riemanniennes, J. Diff. Geom. 13 (1978), 51-78.

[29] F.H. Lin, Analysis on singular spaces, Geometry, Analysis, and Mathematical Physics, ed. Li Ta-Tsien, World Sci. Publications, 1997, pp. 114-126.

[30] C. Mese, Uniqueness theorems for harmonic maps into metric spaces, Commun. Contemp. Math. 4 (2002), 725-750.

[31] A. Papadopoulos, Metric spaces, convexity, and nonpositive curvature, European Math. Soc., Zürich, 2005.

[32] F. Riesz and Sz.-Nagy, Leçons d'Analyse Fonctionnelle, Acad. Sci. Hongrie, Budapest, 1952.

[33] J. Sacks and K. Uhlenbeck, The existence of minimal immersions of 2-spheres, Ann. Math. 113 (1981), 1-24.

[34] R.M. Schoen, Analytic aspects of the harmonic map problem, Conf. Proc. and Lecture Notes in Geometry and Topology, Vol. II, Internat. Press, 1997, 155-203. Earlier version in MSRI Publ. Vol 2, Cambridge, MA, 1984, 321-358.

[35] T. Serbinowski, Boundary regularity of harmonic maps to nonpositively curved metric spaces, Commun. Anal. Geom. 2 (1994), 1-15.

[36] Hölder, Harmonic maps into metric spaces with curvature bounded above, Thesis, Univ. Utah, 1995.

[37] E.H. Spanier, Algebraic topology, McGraw-Hill, New York, 1966.

[38] K.T. Sturm, A semigroup approach to harmonic maps, Potential Anal. 23 (2005), 225-277. 
[39] R. Schoen and S.T. Yau, Harmonic maps and the topology of stable hypersurfaces and manifolds of non-negative Ricci curvature, Comm. Math. Helv. 51 (1976), 333-341.

[40] - Existence of incompressible minimal surfaces and the topology of three dimensional manifolds with non-negative scalar curvature, Ann. Math. 110 (1979), 127-142.

[41] L.-F. Tam, Liouville properties of harmonic maps, Math. Res. Lett. 2 (1995), 719-735.

[42] J.H.C. Whitehead, Simplicial spaces, nuclei, and m-groups, Proc. London Math. Soc. 45 (1939), 243-327.

[43] B. White, Infima of energy functionals in homotopy classes of mappings, J. Diff. Geom. 23 (1986), 127-142.

Department of Mathematics

UNIVERSITETSPARKEN 5

DK-2100 Copenhagen $\varnothing$

DENMARK

E-mail address: Fuglede@math.ku.dk

Received March 29, 2006 
